TRANSACTIONS OF THE

AMERICAN MATHEMATICAL SOCIETY

Volume 353, Number 5, Pages 1839-1883

S 0002-9947(01)02627-7

Article electronically published on January 4, 2001

\title{
SEMI-DUALIZING COMPLEXES AND THEIR AUSLANDER CATEGORIES
}

\author{
LARS WINTHER CHRISTENSEN
}

\begin{abstract}
Let $R$ be a commutative Noetherian ring. We study $R$-modules, and complexes of such, with excellent duality properties. While their common properties are strong enough to admit a rich theory, we count among them such, potentially, diverse objects as dualizing complexes for $R$ on one side, and on the other, the ring itself. In several ways, these two examples constitute the extremes, and their well-understood properties serve as guidelines for our study; however, also the employment, in recent studies of ring homomorphisms, of complexes "lying between" these extremes is incentive.
\end{abstract}

\section{INTRODUCTION}

In this paper all rings are commutative and Noetherian, in particular, $R$ always denotes such a ring. We study $R$-complexes (that is, complexes of $R$-modules) with certain excellent duality properties. The canonical example is the ring itself, considered as a complex concentrated in degree zero. Another example is dualizing complexes for $R$; these were introduced by A. Grothendieck in [RAD and have proved to be a powerful tool, as demonstrated by P. Roberts in [PRb76], and by C. Peskine and L. Szpiro in [CPsLSz73]. Modules with excellent duality properties have been studied by H.-B. Foxby in [HBF72, and in EGl84] by E.S. Golod, who used the name suitable 1 for these modules. Other complexes of the kind considered here were used tacitly by L.L. Avramov and H.-B. Foxby in their study of homological properties of ring homomorphisms [LLAHBF98], and consistently in the ensuing paper [LLAHBF97; in the latter the name relative dualizing complexes was used. This paper offers a generalized and unified treatment of some of the notions and techniques studied and applied in [LLAHBF98, [LLAHBF97, and [EG184]; it provides a common language for stating and proving not only generalizations of results from these papers, but also new results.

The common language developed here is that of semi-dualizing complexes. To illustrate the idea, we start by looking at the modules among these complexes:

A semi-dualizing module for $R$ is a finite (that is, finitely generated) $R$-module $C$ with $\operatorname{Hom}_{R}(C, C)$ canonically isomorphic to $R$ and $\operatorname{Ext}_{R}^{i}(C, C)=0$ for $i>0$. It is not obvious that a local ring may posses semi-dualizing modules other than itself and, possibly, a dualizing module. This was put forward as a question in 1985 by E.S. Golod, see EGl85, and in 1987 H.-B. Foxby gave examples of rings with three different semi-dualizing modules. As a spin-off to the results established here for

Received by the editors January 10, 1999 and, in revised form, March 9, 1999.

1991 Mathematics Subject Classification. Primary 13D25,13C15; Secondary 13D05,13H10.

${ }^{1}$ The paper is in Russian and uses the word удобный, which allows several translations. 
semi-dualizing complexes, we will be able to describe a procedure for constructing Cohen-Macaulay local rings with any finite number of semi-dualizing modules; this is done in section 7 .

Semi-dualizing complexes are defined — in section[2- by generalizing the above definition to the derived category of $R$-modules.

Now, which are the generalized notions and techniques?

The Gorenstein dimension, or G-dimension, for finite modules was introduced by M. Auslander in [MAu67] and developed in MAuMBr69; two of its main features are that it is a finer invariant than the projective dimension and that satisfies an equality of the Auslander-Buchsbaum type. In [SYs95] S. Yassemi studied Gorenstein dimension for complexes through a consistent use of the functor $\mathbf{R H o m} R(-, R)$ and the related category $\mathcal{R}(R)$.

In section 3 we study the functor $\mathbf{R H o m}_{R}(-, C)$ and the related category ${ }_{C} \mathcal{R}(R)$ for a semi-dualizing complex $C$, and we show that it is possible to define a $\mathrm{G}$ dimension with respect to $C$, sharing the nice properties of Auslander's classical $\mathrm{G}$-dimension. That is, there is an inequality:

$$
G-\operatorname{dim}_{C} M \leq \operatorname{pd}_{R} M
$$

for all finite $R$-modules $M$, and equality holds if $\operatorname{pd}_{R} M<\infty$. Furthermore,

$$
G-\operatorname{dim}_{C} M=\operatorname{depth} R-\operatorname{depth}_{R} M
$$

for finite modules with $G-\operatorname{dim}_{C} M<\infty$.

Recall that a finite module is said to be perfect if its projective dimension equals its grade, and Cohen-Macaulay if its Krull dimension equals its depth. Perfectness and Cohen-Macaulayness capture qualitative properties of the module, and perfect modules and Cohen-Macaulay modules have always been considered close kin; indeed, a module of finite projective dimension over a Cohen-Macaulay ring is perfect if and only if it is Cohen-Macaulay.

As it is usual in modern algebra, numerical invariants are introduced to measure 'how far' a module is from possessing such qualitative properties. Our study will make it clear that the invariants imperfection, $\operatorname{imp}_{R} M=\operatorname{pd}_{R} M-\operatorname{grade}_{R} M$, and Cohen-Macaulay defect, $\operatorname{cmd}_{R} M=\operatorname{dim}_{R} M-\operatorname{depth}_{R} M$, for finite $R$-modules $M$, are close kin and in fact, by $(\sharp)$ below, coincide for modules of finite projective dimension over a Cohen-Macaulay ring. Actually, the truly related invariants are the Cohen-Macaulay defect and the quasi-imperfection, cf. [HBF75], and they coincide for modules of finite Gorenstein dimension (in the sense of [MAu67]).

The study of ${ }_{C} \mathcal{R}(R)$ also provides us with valuable information about the semidualizing complex $C$. It turns out that the Cohen-Macaulay defect of $R$ is an upper bound for the Cohen-Macaulay defect as well as the amplitude (homological size) of $C$, and a lower bound for the sum of these invariants. These results are special cases of inequalities like the following:

$$
\operatorname{amp}\left(\mathbf{R H o m}_{R}(M, C)\right) \leq \operatorname{cmd}_{R} M \leq \operatorname{amp}(\mathbf{R H o m} R(M, C))+\operatorname{cmd}_{R} C ;
$$

it holds for modules with $G-\operatorname{dim}_{C} M<\infty$. The homological formula $\operatorname{cmd}_{R} M=$ $\operatorname{amp}\left(\mathbf{R H o m}_{R}(M, D)\right)$, which holds for finite modules $M$ when $D$ is a dualizing complex for $R$, is also contained in $(\sharp)$.

It was discovered in LLAHBF98 that important properties of a local ring homomorphism $\varphi: R \rightarrow S$ of finite flat (Tor-) dimension are reflected in the way $\varphi$ base changes a possible dualizing complex for the source ring $R$. And in [LLAHBF97. 
the larger class of homomorphisms of finite Gorenstein dimension was studied via associated semi-dualizing complexes for the target ring $S$. We pick up this track in sections 5 and 6 and ask the question, 'When does a semi-dualizing complex for the source ring, in a natural way, induce a semi-dualizing complex for the target ring?'

For finite local homomorphisms an answer can be neatly phrased in terms of the generalized $\mathrm{G}$-dimension:

Theorem. Let $C$ be a semi-dualizing complex for $R$. The complex $A=\mathbf{R H o m}_{R}(S, C)$ is semi-dualizing for $S$ if and only if $G-\operatorname{dim}_{C} S<\infty$; and when this is the case, the equality

$$
G-\operatorname{dim}_{C} N=G-\operatorname{dim}_{C} S+G-\operatorname{dim}_{A} N
$$

holds for all finite $S$-modules $N$.

This result represents a vast extension and generalization of [EGl84, Proposition $5]$.

Also for non-finite homomorphisms the answer is related to the concept of Gdimension. To see this we introduce - in section $4-$ two categories, the socalled Auslander and Bass classes ${ }_{C} \mathcal{A}(R)$ and ${ }_{C} \mathcal{B}(R)$, for a semi-dualizing complex $C$, and we show that the functors $C \otimes_{R}^{\mathrm{L}}-$ and $\mathbf{R} \operatorname{Hom}_{R}(C,-)$ provide quasiinverse equivalences of these categories. For dualizing complexes these categories were introduced in [LLAHBF97, and the described equivalences were named Foxby duality in EJX96]. We establish a link to G-dimension by proving (essentially) that finite modules in an Auslander class will have finite G-dimension with respect to some semi-dualizing complex.

In general, by base changing a semi-dualizing complex $C$ for the source ring $R$ we obtain a semi-dualizing complex for the target ring $S$, if and only if $S$ belongs to the Auslander class ${ }_{C} \mathcal{A}(R)$. Every Auslander class will contain all $R$-modules of finite flat dimension, and we can prove more detailed results for homomorphisms of finite flat dimension, along with a variety of ascent and descent results. The general base change result also establishes a converse to the key result in [LLAHBF97] on existence of relative dualizing complexes.

Together, $\mathcal{R}, \mathcal{A}$, and $\mathcal{B}$ are known as Auslander categories, and the underlying idea of this paper is to study semi-dualizing complexes via their Auslander categories - hence the title. This idea is distinctly present in section 8 , there we show how a local ring $R$ and a dualizing complex for $R$ can be distinguished from other semi-dualizing complexes for $R$ by special properties of the functors and categories studied in the previous sections. We also prove that (up to isomorphism and shift) $R$ is the only semi-dualizing complex of finite Gorenstein dimension (in the sense of [MAu67]); and the well-known result that a semi-dualizing complex of finite injective dimension (a dualizing complex) is unique, is sharpened as we show that a semi-dualizing complex of finite Gorenstein injective dimension (in the sense of $[\mathrm{HBF}]$ ) is unique, and in fact dualizing.

I avail myself of this opportunity to thank my supervisor 2 Professor H.-B. Foxby for many valuable discussions concerning the material presented here.

${ }^{2}$ The author is a Ph.D.-student at the University of Copenhagen. 


\section{Homological Algebra for Complexes}

In this paper results are stated and proved in the derived category of the category of $R$-modules. In this first section we recall the vocabulary and some basic, but important, results.

First, a few conventions: All rings are commutative, Noetherian and non-trivial. A ring $R$ is said to be local if it has a unique maximal ideal $\mathfrak{m}$, and we denote the $\mathfrak{m}$-adic completion by $\widehat{R}$. Applied to modules the word finite means finitely generated.

By the flat dimension of a homomorphism of rings $\varphi: R \rightarrow S$ we understand the flat (Tor-) dimension of $S$ (with the imposed structure) over $R$; in particular, we say that $\varphi$ is (faithfully) flat if it makes $S$ a (faithfully) flat $R$-module. We call $\varphi$ finite if it makes $S$ a finite $R$-module, and we say that $\varphi$ is local if $R$ and $S$ are local rings and $\varphi(\mathfrak{m}) \subseteq \mathfrak{n}$, where $\mathfrak{m}$ and $\mathfrak{n}$ are the maximal ideals of $R$ and $S$.

(1.1) Complexes. An $R$-complex $X$ is a sequence of $R$-modules $X_{i}$ and $R$-linear maps $\partial_{i}^{X}: X_{i} \rightarrow X_{i-1}, i \in \mathbb{Z}$. The module $X_{i}$ is called the module in degree $i$, and $\partial_{i}^{X}$ is the $i$-th differential; composition of two consecutive differentials always yields the zero map, i.e., $\partial_{i}^{X} \partial_{i+1}^{X}=0$. If $X_{i}=0$ for $i \neq 0$ we identify $X$ with the module in degree 0 , and an $R$-module $M$ is thought of as a complex $0 \rightarrow M \rightarrow 0$, with $M$ in degree 0 . When $m$ is an integer we denote by $\Sigma^{m} X$ the complex $X$ shifted $m$ degrees (to the left); it is given by $\left(\Sigma^{m} X\right)_{i}=X_{i-m}$ and $\partial_{i}^{\Sigma^{m} X}=(-1)^{m} \partial_{i-m}^{X}$.

The homological position and size of a complex is captured by the numbers supremum, infimum, and amplitude defined by

$$
\begin{aligned}
\sup X & =\sup \left\{i \in \mathbb{Z} \mid \mathrm{H}_{i}(X) \neq 0\right\} ; \\
\inf X & =\inf \left\{i \in \mathbb{Z} \mid \mathrm{H}_{i}(X) \neq 0\right\} ; \text { and } \\
\operatorname{amp} X & =\sup X-\inf X .
\end{aligned}
$$

By convention $\sup X=-\infty$ and $\inf X=\infty$ if $X \simeq 0$.

A morphism $\alpha: X \rightarrow Y$ of $R$-complexes is a sequence of $R$-linear maps $\alpha_{i}$ : $X_{i} \rightarrow Y_{i}$ satisfying $\partial_{i}^{Y} \alpha_{i}-\alpha_{i-1} \partial_{i}^{X}=0$ for $i \in \mathbb{Z}$. We say that a morphism is a quasi-isomorphism if it induces an isomorphism in homology. The symbol $\simeq$ is used to indicate quasi-isomorphisms while $\cong$ indicates isomorphisms of complexes (and hence modules).

(1.2) Derived Category. The derived category of the category of $R$-modules is the category of $R$-complexes localized at the class of all quasi-isomorphisms (see JVe77] and [RAD]), it is denoted by $\mathcal{D}(R)$. We use the symbol $\simeq$ for isomorphisms in $\mathcal{D}(R)$; this is in line with the notation introduced above, as a morphism of complex $\mathrm{s}$ is a quasi-isomorphism exactly if it represents an isomorphism in the derived category. The symbol $\sim$ indicates isomorphism up to shift.

The full subcategories $\mathcal{D}_{+}(R), \mathcal{D}_{-}(R), \mathcal{D}_{\mathrm{b}}(R)$, and $\mathcal{D}_{0}(R)$ consist of complexes $X$ with $\mathrm{H}_{i}(X)=0$ for, respectively, $i \ll 0, i \gg 0,|i| \gg 0$, and $i \neq 0$. By $\mathcal{D}^{\mathrm{f}}(R)$ we denote the full subcategory of $\mathcal{D}(R)$ consisting of complexes $X$ with $\mathrm{H}_{i}(X)$ a finite $R$-module for all $i \in \mathbb{Z}$. For a subcategory $\mathcal{S}(R) \subseteq \mathcal{D}(R)$ we set $\mathcal{S}^{\mathrm{f}}(R)=$ $\mathcal{S}(R) \cap \mathcal{D}^{\mathrm{f}}(R), \mathcal{S}_{0}(R)=\mathcal{S}(R) \cap \mathcal{D}_{0}(R)$, etc. The category of $R$-modules, respectively, finite $R$-modules, is naturally identified with $\mathcal{D}_{0}(R)$, respectively, $\mathcal{D}_{0}^{\mathrm{f}}(R)$. 
(1.3) Derived Functors. The left derived functor of the tensor product functor of $R$-complexes is denoted by $-\otimes_{R}^{\mathbf{L}}-$, and $\mathbf{R} \operatorname{Hom}_{R}(-,-)$ denotes the right derived functor of the homomorphism functor of complexes; by [NSp88 and [LLAGBF91] no boundedness conditions are needed on the arguments. That is, for $X, Y \in \mathcal{D}(R)$ the complexes $X \otimes_{R}^{\mathbf{L}} Y$, and $\mathbf{R H o m}_{R}(X, Y)$ are uniquely determined up to isomorphism in $\mathcal{D}(R)$, and they have the usual functorial properties. For $i \in \mathbb{Z}$ we set $\operatorname{Tor}_{i}^{R}(X, Y)=\mathrm{H}_{i}\left(X \otimes_{R}^{\mathbf{L}} Y\right)$ and $\operatorname{Ext}_{R}^{i}(X, Y)=\mathrm{H}_{-i}\left(\mathbf{R} \operatorname{Hom}_{R}(X, Y)\right)$. For modules $X$ and $Y$ this agrees with the notation of classical homological algebra, so no confusion arises.

Let $\mathfrak{p} \in \operatorname{Spec} R$; by LLAGBF91, 5.2] there are isomorphisms $\left(X \otimes_{R}^{\mathbf{L}} Y\right)_{\mathfrak{p}} \simeq$ $X_{\mathfrak{p}} \otimes_{R_{\mathfrak{p}}}^{\mathbf{L}} Y_{\mathfrak{p}}$ and $\mathbf{R H o m}_{R}(Z, Y)_{\mathfrak{p}} \simeq \mathbf{R H o m}_{R_{\mathfrak{p}}}\left(Z_{\mathfrak{p}}, Y_{\mathfrak{p}}\right)$ in $\mathcal{D}\left(R_{\mathfrak{p}}\right)$. The first one always holds, and the second holds when $Y \in \mathcal{D}_{-}(R)$ and $Z \in \mathcal{D}_{+}^{\mathrm{f}}(R)$. We use these isomorphisms without further comment.

When $R \rightarrow R^{\prime}$ is a homomorphism of rings the following hold [LLAHBF97. (1.2.1) and (1.2.2)]:

(1.3.1) If $X \in \mathcal{D}_{+}^{\mathrm{f}}(R)$ and $X^{\prime} \in \mathcal{D}_{+}^{\mathrm{f}}\left(R^{\prime}\right)$, then $X \otimes_{R}^{\mathrm{L}} X^{\prime} \in \mathcal{D}_{+}^{\mathrm{f}}\left(R^{\prime}\right)$.

(1.3.2) If $X \in \mathcal{D}_{+}^{\mathrm{f}}(R)$ and $Y^{\prime} \in \mathcal{D}_{-}^{\mathrm{f}}\left(R^{\prime}\right)$, then $\operatorname{RHom}_{R}\left(X, Y^{\prime}\right) \in \mathcal{D}_{-}^{\mathrm{f}}\left(R^{\prime}\right)$.

The next two inequalities hold for $X, Z \in \mathcal{D}_{+}(R)$ and $Y \in \mathcal{D}_{-}(R)$, cf. HBF77. (2.1)].

$$
\begin{aligned}
\sup \left(\mathbf{R H o m}_{R}(X, Y)\right) & \leq \sup Y-\inf X ; \text { and } \\
\inf \left(X \otimes_{R}^{\mathbf{L}} Z\right) & \geq \inf X+\inf Z .
\end{aligned}
$$

If $R$ is local, equality holds in the latter when $X, Z \in \mathcal{D}_{+}^{\mathrm{f}}(R)$. This is Nakayama's Lemma for complexes.

(1.4) Homological Dimensions. A complex $X \in \mathcal{D}_{\mathrm{b}}(R)$ is said to be of finite projective (respectively, injective or flat) dimension if $X \simeq U$, where $U$ is a complex of projective (respectively, injective or flat) modules and $U_{i}=0$ for $|i| \gg 0$. By $\mathcal{P}(R), \mathcal{I}(R)$, and $\mathcal{F}(R)$ we denote the full subcategories of $\mathcal{D}_{\mathrm{b}}(R)$ consisting of complexes of, respectively, finite projective, injective, or flat dimension. Note that $\mathcal{P}_{0}(R), \mathcal{I}_{0}(R)$, and $\mathcal{F}_{0}(R)$ are equivalent, respectively, to the full subcategories of modules of finite projective, injective or flat dimension. We use two-letter abbreviations (pd, id, fd) for the homological dimensions.

If $X$ belongs to $\mathcal{D}_{\mathrm{b}}(R)$, then so do the complexes $F \otimes_{R}^{\mathbf{L}} X, \mathbf{R H o m}_{R}(X, I)$, and $\mathbf{R H o m}_{R}(P, X)$ when $F \in \mathcal{F}(R), I \in \mathcal{I}(R)$, and $P \in \mathcal{P}(R)$. To be specific, there are inequalities [LLAGBF91, 2.4.F,I, and P]:

$$
\begin{aligned}
\sup \left(F \otimes_{R}^{\mathbf{L}} X\right) & \leq \mathrm{fd}_{R} F+\sup X ; \\
\inf \left(\mathbf{R} \operatorname{Hom}_{R}(X, I)\right) & \geq-\sup X-\operatorname{id}_{R} I ; \quad \text { and } \\
\inf \left(\mathbf{R H o m}_{R}(P, X)\right) & \geq \inf X-\operatorname{pd}_{R} P .
\end{aligned}
$$

Let $R$ be a local ring with residue field $k$. For $Z \in \mathcal{D}_{\mathrm{b}}^{\mathrm{f}}(R)$ the next equalities hold by LLAGBF91, 2.10.F and 5.5]; note that $\mathcal{P}^{\mathrm{f}}(R)=\mathcal{F}^{\mathrm{f}}(R)$.

$$
\begin{aligned}
\operatorname{pd}_{R} Z & =\operatorname{fd}_{R} Z=\sup \left(Z \otimes_{R}^{\mathbf{L}} k\right) ; \quad \text { and } \\
\operatorname{id}_{R} Z & =-\inf \left(\operatorname{RHom}_{R}(k, Z)\right) .
\end{aligned}
$$


(1.5) Canonical Morphisms. When $R \rightarrow R^{\prime}$ is a homomorphism of rings, a number of canonical homomorphisms in $\mathcal{D}\left(R^{\prime}\right)$ are associated with complexes $X^{\prime}, Y^{\prime}, Z^{\prime}$ $\in \mathcal{D}\left(R^{\prime}\right)$ and $Y, Z \in \mathcal{D}(R)$. We consider the associativity and adjointness isomorphisms:

$$
\begin{aligned}
\left(Z \otimes_{R}^{\mathbf{L}} Y^{\prime}\right) \otimes_{R^{\prime}}^{\mathbf{L}} X^{\prime} & \simeq Z \otimes_{R}^{\mathbf{L}}\left(Y^{\prime} \otimes_{R^{\prime}}^{\mathbf{L}} X^{\prime}\right) ; \\
\mathbf{R H o m}_{R^{\prime}}\left(Z \otimes_{R}^{\mathbf{L}} X^{\prime}, Y^{\prime}\right) & \simeq \mathbf{R H o m}_{R}\left(Z, \mathbf{R H o m}_{R^{\prime}}\left(X^{\prime}, Y^{\prime}\right)\right) ; \quad \text { and } \\
\mathbf{R H o m}_{R}\left(Z^{\prime} \otimes_{R^{\prime}}^{\mathbf{L}} X^{\prime}, Y\right) & \simeq \mathbf{R H o m}_{R^{\prime}}\left(Z^{\prime}, \mathbf{R H o m}_{R}\left(X^{\prime}, Y\right)\right) .
\end{aligned}
$$

These standard isomorphisms are usually used without comment, and so is commutativity of the derived tensor product: $X \otimes_{R}^{\mathbf{L}} Y \simeq Y \otimes_{R}^{\mathbf{L}} X$. The following special cases of the standard isomorphisms are often very useful:

$$
\begin{aligned}
\left(Z \otimes_{R}^{\mathbf{L}} R^{\prime}\right) \otimes_{R^{\prime}}^{\mathbf{L}} X^{\prime} & \simeq Z \otimes_{R}^{\mathbf{L}} X^{\prime} ; \\
\mathbf{R H o m}_{R^{\prime}}\left(Z \otimes_{R}^{\mathbf{L}} R^{\prime}, Y^{\prime}\right) & \simeq \mathbf{R H o m} \\
R & \left(Z, Y^{\prime}\right) ; \quad \text { and } \\
\mathbf{R H o m}_{R^{\prime}}\left(Z^{\prime}, \mathbf{R H o m}_{R}\left(R^{\prime}, Y\right)\right) & \simeq \mathbf{R H o m}_{R}\left(Z^{\prime}, Y\right) .
\end{aligned}
$$

We also consider the evaluation morphisms

$$
\begin{aligned}
& \omega_{Z Y^{\prime} X^{\prime}}: \mathbf{R H o m}_{R}\left(Z, Y^{\prime}\right) \otimes_{R^{\prime}}^{\mathbf{L}} X^{\prime} \rightarrow \mathbf{R H o m}_{R}\left(Z, Y^{\prime} \otimes_{R^{\prime}}^{\mathbf{L}} X^{\prime}\right) ; \quad \text { and } \\
& \theta_{Z X^{\prime} Y^{\prime}}: Z \otimes_{R}^{\mathbf{L}} \mathbf{R H o m}_{R^{\prime}}\left(X^{\prime}, Y^{\prime}\right) \rightarrow \mathbf{R H o m}_{R^{\prime}}\left(\mathbf{R H o m}_{R}\left(Z, X^{\prime}\right), Y^{\prime}\right) .
\end{aligned}
$$

In general, these are not invertible, but by [LLAGBF91, 4.4] the following hold when $Z \in \mathcal{D}_{+}^{\mathrm{f}}(R)$ :

(1.5.7) $\omega_{Z Y^{\prime} X^{\prime}}$ is an isomorphism if $Y^{\prime} \in \mathcal{D}_{-}\left(R^{\prime}\right)$, and $X^{\prime} \in \mathcal{F}\left(R^{\prime}\right)$ or $Z \in \mathcal{P}(R)$.

(1.5.8) $\theta_{Z X^{\prime} Y^{\prime}}$ is an isomorphism if $X^{\prime} \in \mathcal{D}_{\mathrm{b}}\left(R^{\prime}\right)$, and $Y^{\prime} \in \mathcal{I}\left(R^{\prime}\right)$ or $Z \in \mathcal{P}(R)$.

We also use the homothety morphism

$$
\chi_{X}^{R}: R \rightarrow \mathbf{R H o m}_{R}(X, X) ;
$$

and the biduality morphism

$$
\delta_{X}^{Y}: X \rightarrow \mathbf{R H o m}_{R}(\mathbf{R H o m} R(X, Y), Y) .
$$

(1.6) Numerical Invariants. Let $R$ be local with residue field $k$. The depth of an $R$-complex $X$ is defined by

$$
\operatorname{depth}_{R} X=-\sup \left(\mathbf{R H o m}_{R}(k, X)\right) \text {; }
$$

and the (Krull) dimension of $X$ is defined as follows:

$$
\begin{aligned}
\operatorname{dim}_{R} X & =\sup \left\{\operatorname{dim} R / \mathfrak{p}-\inf X_{\mathfrak{p}} \mid \mathfrak{p} \in \operatorname{Spec} R\right\} \\
& =\sup \left\{\operatorname{dim} R / \mathfrak{p}-\inf X_{\mathfrak{p}} \mid \mathfrak{p} \in \operatorname{Supp}_{R} X\right\},
\end{aligned}
$$

where $\operatorname{Supp}_{R} X=\left\{\mathfrak{p} \in \operatorname{Spec} R \mid X_{\mathfrak{p}} \nsucceq 0\right\}=\bigcup_{i \in \mathbb{Z}} \operatorname{Supp}_{R} \mathrm{H}_{i}(X)$. Note that for modules these notions agree with the standard ones.

For $X \not 千 0$ in $\mathcal{D}_{+}(R)$ the inequalities in (1.6.3) and (1.6.5) below follow by the definition of dimension. For $Y \not 0$ in $\mathcal{D}_{-}(R)$ the inequality in (1.6.4) follows by (1.3.3).

$$
\begin{aligned}
& -\infty<-\inf X \leq \operatorname{dim}_{R} X \leq \operatorname{dim} R-\inf X<\infty \\
& -\infty<-\sup Y \leq \operatorname{depth}_{R_{\mathfrak{p}}} Y_{\mathfrak{p}} \quad \text { for } \mathfrak{p} \in \operatorname{Spec} R . \\
& \quad \operatorname{dim}_{R} X \geq \operatorname{dim}_{R_{\mathfrak{p}}} X_{\mathfrak{p}}+\operatorname{dim} R / \mathfrak{p} \quad \text { for } \mathfrak{p} \in \operatorname{Spec} R .
\end{aligned}
$$


Equality holds in (1.6.4) if and only if $\mathfrak{p}$ is associated to the top homology module in $Y$; that is,

$$
\operatorname{depth}_{R_{\mathfrak{p}}} Y_{\mathfrak{p}}=-\sup Y \quad \Longleftrightarrow \mathfrak{p} \in \operatorname{Ass}_{R} \mathrm{H}_{\text {sup } Y}(Y) .
$$

By [HBF79, 3.9 and 2.8] the next inequality holds for $X \in \mathcal{D}_{\mathrm{b}}(R)$ with $\operatorname{depth}_{R} X$ $<\infty$ :

$$
\operatorname{depth}_{R} X \leq \operatorname{dim}_{R} X .
$$

The Cohen-Macaulay defect of $X \in \mathcal{D}(R)$ is as usual the difference $\operatorname{cmd}_{R} X=$ $\operatorname{dim}_{R} X-\operatorname{depth}_{R} X$; by (1.6.7) it is non-negative for $X \in \mathcal{D}_{\mathrm{b}}(R)$ with $\operatorname{depth}_{R} X<$ $\infty$, in particular, for $X \not \varkappa 0$ in $\mathcal{D}_{\mathrm{b}}^{\mathrm{f}}(R)$.

(1.7) Formal Invariants. Let $R$ be local with residue field $k$. For $X \in \mathcal{D}_{+}^{\mathrm{f}}(R)$ the Betti numbers $\beta_{i}^{R}(X)=\operatorname{rank}_{k} \operatorname{Tor}_{i}^{R}(X, k)$ are finite and vanish for $0 \gg i$, cf. (1.3.1). The formal Laurant series $\mathrm{P}_{X}^{R}(t)=\sum_{i \in \mathbb{Z}} \beta_{i}^{R}(X) t^{i}$ is the so-called Poincaré series of $X$; it has non-negative integer coefficients, and by Nakayama's Lemma and (1.4.4) there are equalities:

$$
\operatorname{ord} \mathrm{P}_{X}^{R}(t)=\inf X \quad \text { and } \quad \operatorname{deg} \mathrm{P}_{X}^{R}(t)=\operatorname{pd}_{R} X .
$$

Furthermore, the following hold:

$$
X \sim R \quad \Longleftrightarrow \quad \mathrm{P}_{X}^{R}(t)=t^{d} \text { for some } d \in \mathbb{Z} .
$$

For $Y \in \mathcal{D}_{-}^{\mathrm{f}}(R)$ the Bass numbers $\mu_{R}^{i}(Y)=\operatorname{rank}_{k} \operatorname{Ext}_{R}^{i}(k, Y)$ are finite and vanish for $i \gg 0$, cf. (1.3.2). The formal Laurant series $\mathrm{I}_{R}^{Y}(t)=\sum_{i \in \mathbb{Z}} \mu_{R}^{i}(Y) t^{i}$ is called the Bass series of $Y$; it has non-negative integer coefficients, and by the definition of depth 1.6.1 and 1.4.5 there are equalities:

$$
\operatorname{ord} \mathrm{I}_{R}^{Y}(t)=\operatorname{depth}_{R} Y \quad \text { and } \quad \operatorname{deg} \mathrm{I}_{R}^{Y}(t)=\operatorname{id}_{R} Y .
$$

For convenience we set $\mu_{R}^{i}=\mu_{R}^{i}(R)$ and $\mathrm{I}_{R}^{R}(t)=\mathrm{I}_{R}(t)$.

Let $Y \in \mathcal{D}_{-}^{\mathrm{f}}(R)$, let $\mathfrak{p} \in \operatorname{Spec} R$, and set $n=\operatorname{dim} R / \mathfrak{p}$. The next implication holds for all $i \in \mathbb{Z}$ (of course, $\mu_{R}^{i}(\mathfrak{p}, Y)$ is the rank of the $R_{\mathfrak{p}} / \mathfrak{p}_{\mathfrak{p}}$-vector space $\left.\operatorname{Ext}_{R_{\mathfrak{p}}}^{i}\left(R_{\mathfrak{p}} / \mathfrak{p}_{\mathfrak{p}}, Y_{\mathfrak{p}}\right)\right)$ :

$$
\mu_{R}^{i}(\mathfrak{p}, Y) \neq 0 \quad \Longrightarrow \quad \mu_{R}^{i+n}(Y) \neq 0 .
$$

This is a complex version of [HBs63, (3.1)], cf. [HHA, Chapter 13], and as for modules the next inequality follows, cf. (1.7.3).

$$
\operatorname{depth}_{R} Y \leq \operatorname{depth}_{R_{\mathfrak{p}}} Y_{\mathfrak{p}}+\operatorname{dim} R / \mathfrak{p} .
$$

Let $\varphi: R \rightarrow R^{\prime}$ be a local homomorphism; the next two equalities of formal Laurant series are established in [LLAHBF97, (1.5.3)].

$$
\mathrm{P}_{X \otimes{ }_{R}^{\prime} X^{\prime}}^{R^{\prime}}(t)=\mathrm{P}_{X}^{R}(t) \mathrm{P}_{X^{\prime}}^{R^{\prime}}(t)
$$

holds for $X \in \mathcal{D}_{+}^{\mathrm{f}}(R)$ and $X^{\prime} \in \mathcal{D}_{+}^{\mathrm{f}}\left(R^{\prime}\right)$; and

$$
\mathrm{I}_{R^{\prime}}^{\mathrm{RHom}} \operatorname{Hom}_{R}\left(X, Y^{\prime}\right)(t)=\mathrm{P}_{X}^{R}(t) \mathrm{I}_{R^{\prime}}^{Y^{\prime}}(t)
$$

holds for $X \in \mathcal{D}_{+}^{\mathrm{f}}(R)$ and $Y^{\prime} \in \mathcal{D}_{-}^{\mathrm{f}}\left(R^{\prime}\right)$. We shall also need the following: 
(1.7.8) Lemma. Let $\varphi: R \rightarrow R^{\prime}$ be a finite local homomorphism. If $Y \in \mathcal{D}_{-}^{\mathrm{f}}(R)$ and $X^{\prime} \in \mathcal{D}_{+}^{\mathrm{f}}\left(R^{\prime}\right)$, then $\mathbf{R H o m}_{R}\left(X^{\prime}, Y\right)$ belongs to $\mathcal{D}_{-}^{\mathrm{f}}\left(R^{\prime}\right)$, and there is an equality of formal Laurant series:

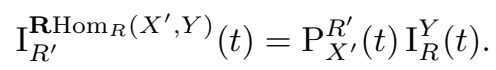

Proof. The $R^{\prime}$-structure, and thereby the $R$-structure, of $\mathbf{R H o m}_{R}\left(X^{\prime}, Y\right)$ is determined by $X^{\prime}$. Since $\varphi$ is finite $X^{\prime}$ belongs to $\mathcal{D}_{+}^{\mathrm{f}}(R)$, so $\operatorname{RHom}_{R}\left(X^{\prime}, Y\right) \in \mathcal{D}_{-}^{\mathrm{f}}(R)$ by (1.3.2) and hence $\mathbf{R H o m}_{R}\left(X^{\prime}, Y\right) \in \mathcal{D}_{-}^{\mathrm{f}}\left(R^{\prime}\right)$ as desired. We denote the residue fields of $R$ and $R^{\prime}$ by, respectively, $k$ and $k^{\prime}$; using that $\varphi$ is local we find that

$$
\begin{aligned}
\operatorname{RHom}_{R^{\prime}}\left(k^{\prime}, \operatorname{RHom}_{R}\left(X^{\prime}, Y\right)\right) & \simeq \mathbf{R H o m}_{R}\left(k^{\prime} \otimes_{R^{\prime}}^{\mathbf{L}} X^{\prime}, Y\right) \\
& \simeq \mathbf{R H o m}_{R}\left(\left(X^{\prime} \otimes_{R^{\prime}}^{\mathbf{L}} k^{\prime}\right) \otimes_{k} k, Y\right) \\
& \simeq \operatorname{Hom}_{k}\left(X^{\prime} \otimes_{R^{\prime}}^{\mathbf{L}} k^{\prime}, \mathbf{R H o m}_{R}(k, Y)\right) .
\end{aligned}
$$

Hence we have $\operatorname{Ext}_{R^{\prime}}^{*}\left(k^{\prime}, \mathbf{R H o m}_{R}\left(X^{\prime}, Y\right)\right) \cong \operatorname{Hom}_{k}\left(\operatorname{Tor}_{*}^{R^{\prime}}\left(X^{\prime}, k^{\prime}\right), \operatorname{Ext}_{R}^{*}(k, Y)\right)$, and the equality of Laurant series follows.

(1.8) Dualizing Complexes. Let $R$ be a local ring. Recall that an $R$-complex $D$ is said to be dualizing for $R$ if and only if $D \in \mathcal{I}^{\mathrm{f}}(R)$ and the homothety morphism $\chi_{D}^{R}: R \rightarrow \mathbf{R H o m}_{R}(D, D)$ is invertible. The following hold [RAD, V.3.4]:

$$
D \text { is dualizing for } R \quad \Longleftrightarrow \quad \mathrm{I}_{R}^{D}(t)=t^{d} \text { for some } d \in \mathbb{Z} \text {. }
$$

We say that $D$ is a normalized dualizing complex for $R$ if $\mathrm{I}_{R}^{D}(t)=1$. This is in keeping with the convention used in [LLAHBF97].

Not all rings have a dualizing complex, but an ample supply of rings that do is ensured by the following:

(1.8.2) $R$ is Gorenstein if and only if the $R$-module $R$ is a dualizing complex for $R$ RAD V.3.4 and V.10].

(1.8.3) Every homomorphic image of a Gorenstein local ring has a dualizing complex. In particular, every complete local ring has a dualizing complex RAD. V.10].

\section{Dagger Duality}

In this section we define semi-dualizing complexes and collect some technical results for later reference. We also introduce the first Auslander category - the category of reflexive complexes - and its related duality functor.

(2.1) Definition. An $R$-complex $C$ is said to be semi-dualizing for $R$ if and only if $C \in \mathcal{D}_{\mathrm{b}}^{\mathrm{f}}(R)$ and the homothety morphism $\chi_{C}^{R}: R \rightarrow \mathbf{R} \operatorname{Hom}_{R}(C, C)$ is an isomorphism.

(2.2) Remark. If $R$ is local, then a complex $D \in \mathcal{D}(R)$ is dualizing for $R$ if and only if it is semi-dualizing and of finite injective dimension, cf. (1.8).

(2.3) Examples. The canonical example of a semi-dualizing complex for $R$ is the ring itself; other examples are dualizing complexes, when these exist, and relative dualizing complexes, cf. [LLAHBF97]. More examples of semi-dualizing complexes are given in section 7 . 
(2.4) Observation. If $C$ is a semi-dualizing complex for $R$, then so are all the shifted complexes $\Sigma^{n} C, n \in \mathbb{Z}$.

The next two results on localization and completion are easily derived from, respectively, (5.1) and (5.6). Since we draw heavily on the first one in section 3 , we have included the straightforward proof.

(2.5) Lemma. If $C$ is a semi-dualizing complex for $R$, then $\operatorname{Supp}_{R} C=\operatorname{Spec} R$, and for each $\mathfrak{p} \in \operatorname{Spec} R$ the complex $C_{\mathfrak{p}}$ is semi-dualizing for $R_{\mathfrak{p}}$.

Proof. Let $\mathfrak{p} \in \operatorname{Spec} R$. The commutative diagram

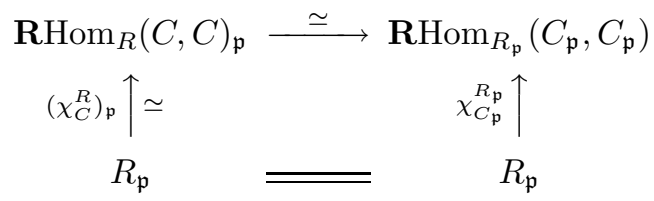

shows that $C_{\mathfrak{p}}$ is semi-dualizing for $R_{\mathfrak{p}}$. In particular, it follows that $\operatorname{Supp}_{R} C=$ Spec $R$ as claimed.

(2.6) Lemma. Let $R$ be a local ring. A complex $C \in \mathcal{D}_{\mathrm{b}}^{\mathrm{f}}(R)$ is semi-dualizing for $R$ if and only if the complex $C \otimes_{R} \widehat{R} \in \mathcal{D}(\widehat{R})$ is semi-dualizing for $\widehat{R}$.

(2.7) Definitions. Let $C$ be a semi-dualizing complex for $R$. For $Z \in \mathcal{D}(R)$ the dagger dual with respect to $C$ is the complex $Z^{\dagger_{C}}=\mathbf{R H o m}_{R}(Z, C)$, and - t$_{C}=$ $\mathbf{R H o m}_{R}(-, C)$ is the corresponding dagger duality functor. For convenience we set $Z^{\dagger_{C} \dagger_{C}}=\left(Z^{\dagger_{C}}\right)^{\dagger_{C}}$.

An $R$-complex $Z$ is said to be $C$-reflexive if and only if $Z$ and the dagger dual $Z^{\dagger_{C}}$ belong to $\mathcal{D}_{\mathrm{b}}^{\mathrm{f}}(R)$, and the biduality morphism $\delta_{Z}^{C}: Z \rightarrow Z^{\dagger_{C} \dagger_{C}}$ is invertible. By ${ }_{C} \mathcal{R}(R)$ we denote the full subcategory of $\mathcal{D}_{\mathrm{b}}^{\mathrm{f}}(R)$ whose objects are the $C$-reflexive complexes.

(2.8) Remarks. It is straightforward to check that $R$ and $C$ belong to ${ }_{C} \mathcal{R}(R)$ when $C$ is semi-dualizing for $R$. By [SYs95, 2.7 and 2.8] the complexes in ${ }_{R} \mathcal{R}(R)$ are exactly those of finite Gorenstein dimension; and it is well-known that ${ }_{D} \mathcal{R}(R)=$ $\mathcal{D}_{\mathrm{b}}^{\mathrm{f}}(R)$ if $R$ is local and $D$ is a dualizing complex for $R$, cf. [RAD, V.2.1].

A word of caution: An $R$-module $M$ with the property that the canonical map $\varepsilon_{M}: M \rightarrow \operatorname{Hom}_{R}\left(\operatorname{Hom}_{R}(M, R), R\right)$ is an isomorphism is sometimes called reflexive. However, such a module need not be $R$-reflexive in the sense defined above, and vice versa: an $R$-reflexive module, in the sense of (2.7), need not have the property. See [SYs95, 2.6] for examples.

(2.9) Proposition. If $C$ is a semi-dualizing complex for $R$, then there is a full embedding:

$$
\mathcal{P}^{\mathrm{f}}(R) \subseteq{ }_{C} \mathcal{R}(R)
$$


Proof. Let $P \in \mathcal{P}^{\mathrm{f}}(R)$, then the complex $P^{\dagger_{C}}$ belongs to $\mathcal{D}_{\mathrm{b}}^{\mathrm{f}}(R)$ and the evaluation morphism $\theta_{P C C}$ is invertible, cf. (1.4.3) and (1.5.8). The commutative diagram

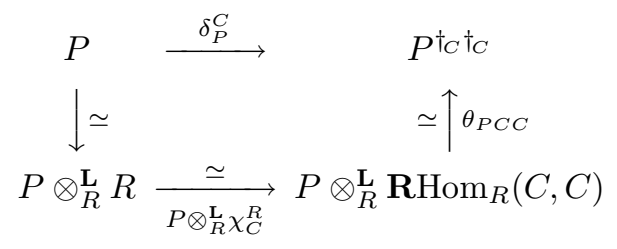

shows that $\delta_{P}^{C}$ is an isomorphism, and hence $P$ is $C$-reflexive.

The behavior of $C$-reflexive complexes under completion and localization is explained by (5.10). Since we shall use it repeatedly in the next section, we spell out the result on localization:

(2.10) Lemma. Let $C$ be a semi-dualizing complex for $R$. The next implication holds for $Z \in \mathcal{D}_{\mathrm{b}}^{\mathrm{f}}(R)$ and $\mathfrak{p} \in \operatorname{Spec} R$ :

$$
Z \in{ }_{C} \mathcal{R}(R) \quad \Longrightarrow \quad Z_{\mathfrak{p}} \in{ }_{C_{\mathfrak{p}}} \mathcal{R}\left(R_{\mathfrak{p}}\right) .
$$

(2.11) Dagger Duality Theorem. Let $C$ be a semi-dualizing complex for $R$. Dagger duality with respect to $C$ is an endofunctor of ${ }_{C} \mathcal{R}(R)$, and it takes semidualizing complexes to semi-dualizing complexes.

Proof. Suppose $Z \in{ }_{C} \mathcal{R}(R)$, then $Z^{\dagger_{C}}$ belongs to $\mathcal{D}_{\mathrm{b}}^{\mathrm{f}}(R)$ and so does $\left(Z^{\dagger_{C}}\right)^{\dagger_{C}} \simeq Z$. The commutative diagram

$$
\begin{array}{cc}
\left(Z^{\dagger_{C}}\right)^{\dagger_{C} \dagger_{C}}=\left(Z^{\dagger_{C} \dagger_{C}}\right)^{\dagger_{C}} \\
\left.\simeq \downarrow_{\delta^{\dagger_{C}}}^{C}\right|^{\left(\delta_{Z}^{C}\right)^{\dagger_{C}}} \\
Z^{\dagger_{C}}=Z^{\dagger_{C}}
\end{array}
$$

shows that the biduality map $\delta_{Z^{\dagger} C}^{C}$ is an isomorphism, and hence $Z^{\dagger_{C}} \in{ }_{C} \mathcal{R}(R)$. Since ${ }_{C} \mathcal{R}(R)$ is a full subcategory, it follows that $-^{\dagger_{C}}$ is an endofunctor of ${ }_{C} \mathcal{R}(R)$.

Suppose $Z \in{ }_{C} \mathcal{R}(R)$ is semi-dualizing for $R$. The dagger dual $Z^{\dagger_{C}}$ belongs to $\mathcal{D}_{\mathrm{b}}^{\mathrm{f}}(R)$, and the diagram

$$
\begin{array}{cc}
R & \stackrel{\chi_{Z}^{R}}{\longrightarrow} \operatorname{RHom}_{R}(Z, Z) \\
\chi_{Z^{\dagger_{C}}}^{R} \downarrow & \mathbf{R} \operatorname{Hom}_{R}\left(Z, \delta_{Z}^{C}\right) \downarrow \simeq \\
\mathbf{R H o m}_{R}\left(Z^{\dagger_{C}}, Z^{\dagger_{C}}\right) \stackrel{\simeq}{\longrightarrow} \mathbf{R H o m}_{R}\left(Z, Z^{\dagger_{C} \dagger_{C}}\right)
\end{array}
$$

where the bottom row isomorphism involves only standard isomorphisms, is commutative. The homothety morphism $\chi_{Z^{\dagger_{C}}}^{R}$ is, therefore, invertible and $Z^{\dagger_{C}}$, indeed, a semi-dualizing complex for $R$.

As mentioned in (2.8) every complex in $\mathcal{D}_{\mathrm{b}}^{\mathrm{f}}(R)$ is $D$-reflexive when $R$ is local and $D$ is dualizing for $R$. Thus, the dagger duality functor $-^{\dagger} D$ is an endofunctor of $\mathcal{D}_{\mathrm{b}}^{\mathrm{f}}(R)$, and we have the following:

(2.12) Corollary. If $R$ is local and $D$ is a dualizing complex for $R$, then $C \in \mathcal{D}_{\mathrm{b}}^{\mathrm{f}}(R)$ is semi-dualizing for $R$ if and only if $C^{\dagger_{D}}$ is so. 
For every semi-dualizing complex $C$ the complexes with finite homology and finite projective dimension form a full subcategory of ${ }_{C} \mathcal{R}(R)$, cf. (2.9). The next theorem shows that for $C=R$ dagger duality is stable on this subcategory, and in section 8 we show that this property distinguishes a local ring $R$ from its other semi-dualizing complexes.

(2.13) Theorem. Dagger duality with respect to $R$ is an endofunctor of $\mathcal{P}^{\mathrm{f}}(R)$, and for $P \in \mathcal{P}^{\mathrm{f}}(R)$ the next two equalities hold.

$$
\operatorname{pd}_{R} P^{\dagger_{R}}=-\inf P \quad \text { and } \quad \inf P^{\dagger_{R}}=\operatorname{pd}_{R} P .
$$

Furthermore, if $R$ is local and $P \in \mathcal{P}^{\mathrm{f}}(R)$, then there is an equality of Poincaré series:

$$
\mathrm{P}_{P^{\dagger} R}^{R}(t)=\mathrm{P}_{P}^{R}\left(t^{-1}\right) .
$$

Proof. Let $P \in \mathcal{P}^{\mathrm{f}}(R)$. By [LLAGBF91, 2.5.P], (1.5.8), and (1.3.4) we have

$$
\begin{aligned}
\operatorname{pd}_{R} P^{\dagger_{R}} & =\sup \left\{-\inf \left(\mathbf{R} \operatorname{Hom}_{R}\left(P^{\dagger_{R}}, N\right)\right) \mid N \in \mathcal{D}_{0}(R)\right\} \\
& =\sup \left\{-\inf \left(P \otimes_{R}^{\mathbf{L}} \mathbf{R} \operatorname{Hom}_{R}(R, N)\right) \mid N \in \mathcal{D}_{0}(R)\right\} \\
& =\sup \left\{-\inf \left(P \otimes_{R}^{\mathbf{L}} N\right) \mid N \in \mathcal{D}_{0}(R)\right\} \\
& =-\inf P .
\end{aligned}
$$

This proves the first equality in (a), and it follows that $-\dagger_{R}$ is an endofunctor of $\mathcal{P}^{\mathrm{f}}(R)$. The second equality in (a) follows by the first one, as $P=P^{\dagger_{R} \dagger_{R}}$ by (2.9).

Now, let $R$ be local with residue field $k$, and let $P \in \mathcal{P}^{\mathrm{f}}(R)$. The equality of Poincaré series, (b), follows by the calculation below, where $\mathrm{E}_{R}(k)$ denotes the injective hull of the residue field. This part of the proof is taken from HHA Chapter 11].

$$
\begin{array}{rlr}
\beta_{i}^{R}\left(P^{\dagger_{R}}\right) & =\operatorname{rank}_{k} \operatorname{Tor}_{i}^{R}\left(P^{\dagger_{R}}, k\right) & \\
& =\operatorname{rank}_{k} \mathrm{H}_{i}\left(\mathbf{R} \operatorname{Hom}_{R}(P, R) \otimes_{R}^{\mathbf{L}} k\right) & \text { by (1.5.7) } \\
& =\operatorname{rank}_{k} \mathrm{H}_{i}\left(\mathbf{R} \operatorname{Hom}_{R}\left(P, R \otimes_{R}^{\mathbf{L}} k\right)\right) & \\
& =\operatorname{rank}_{k} \mathrm{H}_{-i}\left(\operatorname{Hom}_{R}\left(\mathbf{R} \operatorname{Hom}_{R}(P, k), \mathrm{E}_{R}(k)\right)\right) & \\
& =\operatorname{rank}_{k} \mathrm{H}_{-i}\left(P \otimes_{R}^{\mathbf{L}} \operatorname{Hom}_{R}\left(k, \mathrm{E}_{R}(k)\right)\right) & \text { by (1.5.8) } \\
& =\operatorname{rank}_{k} \operatorname{Tor}_{-i}^{R}(P, k) \\
& =\beta_{-i}^{R}(P) . \quad \square
\end{array}
$$

(2.14) Corollary (from [HHA] $)$. Let $R$ be local. For $Z \in \mathcal{D}_{\mathrm{b}}^{\mathrm{f}}(R)$ and $P \in \mathcal{P}^{\mathrm{f}}(R)$ there is an equality of formal Laurant series:

$$
\mathrm{P}_{\mathbf{R} \operatorname{Hom}_{R}(P, Z)}^{R}(t)=\mathrm{P}_{P}^{R}\left(t^{-1}\right) \mathrm{P}_{Z}^{R}(t) .
$$

Proof. If $P \in \mathcal{P}^{\mathrm{f}}(R)$, then $P \simeq P^{\dagger_{R} \dagger_{R}}$ and $P^{\dagger_{R}} \in \mathcal{P}^{\mathrm{f}}(R)$ by (2.9) and (2.13). Let $Z \in \mathcal{D}_{\mathrm{b}}^{\mathrm{f}}(R)$; by $(1.5 .8)$ we have

$$
\begin{aligned}
\mathbf{R H o m}_{R}(P, Z) & \simeq \mathbf{R} \operatorname{Hom}_{R}\left(\mathbf{R H o m}_{R}\left(P^{\dagger_{R}}, R\right), Z\right) \\
& \simeq P^{\dagger_{R}} \otimes_{R}^{\mathbf{L}} \mathbf{R} \operatorname{Hom}_{R}(R, Z) \simeq P^{\dagger_{R}} \otimes_{R}^{\mathbf{L}} Z .
\end{aligned}
$$

The desired equality now follows by (1.7.6) and (2.13)(b). 


\section{Reflexive Complexes And G-Dimension}

Throughout this section $R$ is a local ring.

We make a detailed study of reflexive complexes, focusing on the interplay between dagger duality and the invariants dimension and depth. The essential results are described in (3.3) and (3.8). The study also reveals strong relations between invariants of a ring and those of its semi-dualizing complexes. The essentials in this direction are (3.4) and (3.7).

The opening lemma is central for the study of reflexive complexes; the proof is deferred to the end of the section.

(3.1) Lemma. Let $C$ be a semi-dualizing complex for $R$. If $Z$ is $C$-reflexive, then the following hold:

(a) $\operatorname{depth}_{R} Z-\inf Z^{\dagger_{C}}=\operatorname{depth}_{R} C$;

(b) $\operatorname{depth}_{R} C \leq \operatorname{dim}_{R} Z-\sup Z^{\dagger_{C}} \leq \operatorname{dim}_{R} C$;

(c) $\operatorname{dim}_{R} Z-\sup Z^{\dagger_{C}} \leq \operatorname{dim}_{R} Z^{\dagger_{C}}-\inf Z$; and

(d) $\operatorname{Supp}_{R} Z^{\dagger_{C}}=\operatorname{Supp}_{R} Z$.

(3.2) Corollary. If $C$ is a semi-dualizing complex for $R$, then the following hold:

(a) $\operatorname{depth}_{R} C=\operatorname{depth} R-\inf C$; and

(b) $\operatorname{dim} R-\sup C \leq \operatorname{dim}_{R} C \leq \operatorname{dim} R-\inf C$.

Proof. (a) is immediate by applying (3.1) (a) to $Z=R$. The first inequality in (b) similarly follows by applying (3.1) (b) to $Z=R$ while the second holds in general, cf. 1.6.3.

(3.3) Theorem. Let $C$ be a semi-dualizing complex for $R$. If $Z$ is $C$-reflexive, then the following hold:

(a) $\operatorname{amp} Z^{\dagger_{C}} \leq \operatorname{cmd}_{R} Z \leq \operatorname{amp} Z^{\dagger_{C}}+\operatorname{cmd}_{R} C$; and

(b) $\operatorname{cmd}_{R} Z \leq \operatorname{amp} Z^{\dagger_{C}}+\operatorname{cmd}_{R} Z^{\dagger_{C}}$.

Proof. (a) follows immediately by (a) and (b) in (3.1). To establish (b) we use that also $Z^{\dagger_{C}} \in{ }_{C} \mathcal{R}(R)$ :

$$
\begin{aligned}
\operatorname{cmd}_{R} Z & =\operatorname{dim}_{R} Z-\operatorname{depth}_{R} Z & \\
& \leq \operatorname{dim}_{R} Z^{\dagger_{C}}+\sup Z^{\dagger_{C}}-\inf Z-\operatorname{depth}_{R} Z & \text { by (3.1) (c) } \\
& =\operatorname{dim}_{R} Z^{\dagger_{C}}+\sup Z^{\dagger_{C}}-\inf Z-\operatorname{depth}_{R} C-\inf Z^{\dagger_{C}} & \text { by (3.1) (a) } \\
& =\operatorname{dim}_{R} Z^{\dagger_{C}}+\operatorname{amp} Z^{\dagger_{C}}-\operatorname{depth}_{R} Z^{\dagger_{C}} & \text { by (3.1) (a) } \\
& =\operatorname{amp} Z^{\dagger_{C}}+\operatorname{cod}_{R} Z^{\dagger_{C}} . \quad \square &
\end{aligned}
$$

(3.4) Corollary. If $C$ is a semi-dualizing complex for $R$, then the next three inequalities hold.

(a) $\operatorname{amp} C \leq \operatorname{cmd} R$;

(b) $\operatorname{cmd}_{R} C \leq \mathrm{cmd} R$; and

(c) $\operatorname{cmd} R \leq \operatorname{amp} C+\operatorname{cmd}_{R} C$. 
Equality holds in (a) if $C$ is Cohen-Macaulay and in (b) if $\operatorname{amp} C=0$, i.e., if $C$ is a module up to a shift.

Proof. Applying (3.3) (a) to $Z=R$ yields (a) and (c) while (3.3) (b) applied to $Z=C$ accounts for (b). It follows from (a) and (c) that amp $C=\operatorname{cmd} R$ if $\operatorname{cmd}_{R} C=0$, and similarly it follows from (b) and (c) that $\mathrm{cmd}_{R} C=\operatorname{cmd} R$ if $\operatorname{amp} C=0$.

In the appendix we show how defects in chains of prime ideals obstruct equality in $(3.4)$ (c).

(3.5) Remarks. A dualizing complex $D$ is Cohen-Macaulay: The inequality below follows by (1.7.4) (as for modules, cf. [HBs63, (3.2)]), and the equalities are, respectively, the Bass formula for complexes, cf. [HBF77, 4.1(b) and 4.3(1)], and (3.2) (a).

$$
\operatorname{dim}_{R} D \leq \operatorname{id}_{R} D=\operatorname{depth} R-\inf D=\operatorname{depth}_{R} D .
$$

Thus, $\operatorname{dim}_{R} D=\operatorname{depth}_{R} D$ by (1.6.7), and (3.4) contains the fact that amp $D=$ cmd $R$.

For $Z \in{ }_{R} \mathcal{R}(R)$ the two inequalities in (3.3), applied to $Z^{\dagger_{R}} \in{ }_{R} \mathcal{R}(R)$, read:

$$
\operatorname{cmd}_{R} Z^{\dagger_{R}} \leq \operatorname{amp} Z+\operatorname{cmd} R \quad \text { and } \quad \operatorname{cmd}_{R} Z^{\dagger_{R}} \leq \operatorname{amp} Z+\operatorname{cmd}_{R} Z \text {. }
$$

When $Z$ is a module, the second inequality is known from [SYs95, 2.18]. We note that for $Z \in \mathcal{P}^{\mathrm{f}}(R)$ the first inequality is stronger than the second by the New Intersection Theorem, cf. 3.6.1) below.

(3.6) New Intersection Theorem. Let $R$ be a local ring. The following inequalities hold for $Z \in \mathcal{D}_{\mathrm{b}}^{\mathrm{f}}(R)$ and $P \nsucceq 0$ in $\mathcal{P}^{\mathrm{f}}(R)$ :

$$
\begin{aligned}
\operatorname{cmd}_{R}\left(Z \otimes_{R}^{\mathrm{L}} P\right) & \geq \operatorname{cmd}_{R} Z ; \quad \text { and } \\
\operatorname{amp}\left(Z \otimes_{R}^{\mathrm{L}} P\right) & \geq \operatorname{amp} Z .
\end{aligned}
$$

The first inequality can, cf. [HHA, Chapter 18], be derived from the New Intersection Theorem due to Peskine and Szpiro [CPsLSz73, Hochster MHc75], and Roberts PRb76, PRb87]. The second inequality is Iversen's Amplitude Inequality BIv77, (3.2)]. Its proof uses the New Intersection Theorem; note that it holds without restrictions on $R$ since, with the appearance of [PRb87, the extra requirement of [BIv77] that $R$ be an algebra over a field is no longer needed.

Before we carry on with another important corollary to (3.3), we note that (3.4) offers the following characterization of Cohen-Macaulay rings:

(3.7) Corollary. The next three conditions are equivalent.

(i) $R$ is Cohen-Macaulay.

(ii) $R$ has a Cohen-Macaulay semi-dualizing module.

(iii) Every semi-dualizing complex for $R$ is a maximal Cohen-Macaulay module (up to a shift). 
Proof. $(i) \Rightarrow$ (iii): Suppose $R$ is Cohen-Macaulay and let $C$ be a semi-dualizing complex for $R$. It follows by (a) and (b) in (3.4) that $C$ is Cohen-Macaulay and $\operatorname{amp} C=0$. We may assume that $C$ is concentrated in degree 0 , and then it follows by (3.2) (a) that $\operatorname{dim}_{R} C=\operatorname{depth}_{R} C=\operatorname{depth} R=\operatorname{dim} R$.

The implication $(i i i) \Rightarrow(i i)$ is obvious, and $(i i) \Rightarrow(i)$ follows by (3.4) (c).

The next result is immediate by (3.3)(a). It generalizes the homological formula $\operatorname{cmd}_{R} Z=\operatorname{amp} Z^{\dagger}$, which holds for complexes $Z$ in $\mathcal{D}_{\mathrm{b}}^{\mathrm{f}}(R)={ }_{D} \mathcal{R}(R)$ when $D$ is dualizing for $R$, cf. [HBF79, 3.14].

(3.8) Corollary (CMD Formula). Let $C$ be a Cohen-Macaulay semi-dualizing complex for $R$. If $Z$ is $C$-reflexive, then

$$
\operatorname{cmd}_{R} Z=\operatorname{amp} Z^{\dagger} C
$$

(3.9) (Quasi-)Imperfection. An $R$-module $M$ is said to be perfect if its projective dimension equals its grade, and the integer $\operatorname{imp}_{R} M=\operatorname{pd}_{R} M-\operatorname{grade}_{R} M$ is referred to as the imperfection of $M$. For $M \in \mathcal{P}_{0}^{\mathrm{f}}(R)$ there is an equality $\operatorname{imp}_{R} M=\operatorname{amp} M^{\dagger_{R}}$. With the convention that $\operatorname{imp}_{R} Z=\operatorname{pd}_{R} Z-\operatorname{grade}_{R} Z$ and $\operatorname{grade}_{R} Z=-\sup Z^{\dagger_{R}}$ for $Z$ in $\mathcal{D}_{\mathrm{b}}^{\mathrm{f}}(R)$, cf. [HBF79, 5.3], the equality $\operatorname{imp}_{R} Z=$ $\operatorname{amp} Z^{\dagger_{R}}$ is valid for $Z \in \mathcal{P}^{\mathrm{f}}(R)$.

Replacing the projective dimension by Auslander's G-dimension [MAu67, and MAuMBr69 we obtain the notion of quasi-perfect modules, cf. [HBF75; and the quasi-imperfection, $\mathrm{q}-\operatorname{imp}_{R} M=G-\operatorname{dim}_{R} M-\operatorname{grade}_{R} M$, satisfies the formula q-imp ${ }_{R} M=\operatorname{amp} M^{\dagger_{R}}$, when $G-\operatorname{dim}_{R} M$ is finite. By a result due to Foxby, cf. SYs95, 2.7], a finite $R$-module has finite $\mathrm{G}$-dimension if and only if it belongs to $R \mathcal{R}(R)$; and with the extension of the $\mathrm{G}$-dimension to complexes [SYs95. 2.8 ] the $R$-reflexive complexes are exactly those of finite $\mathrm{G}$-dimension. Moreover, $G-\operatorname{dim}_{R} Z=-\inf Z^{\dagger_{R}}$ for these complexes, and hence

$$
\text { q-imp }{ }_{R} Z=\operatorname{amp} Z^{\dagger_{R}} \text { for } Z \in{ }_{R} \mathcal{R}(R) .
$$

Thus, at least on the formal level of homological formulae, the quasi-imperfection and the Cohen-Macaulay defect are invariants of the same kind. By (3.8) they coincide for complexes of finite G-dimension over a Cohen-Macaulay ring; in particular, they agree for all complexes in $\mathcal{D}_{\mathrm{b}}^{\mathrm{f}}(R)$ when $R$ is Gorenstein, see also (8.5).

The next proposition is an extension of [LLAHBF98, (2.6.2)] to complexes.

(3.10) Proposition. For $Z \in \mathcal{P}^{\mathrm{f}}(R)$ the following inequalities hold:

$$
0 \leq \operatorname{cmd}_{R} Z-\operatorname{cmd} R \leq \operatorname{imp}_{R} Z \leq \operatorname{cmd}_{R} Z .
$$

In particular, the next two conditions are equivalent.

(i) $R$ is Cohen-Macaulay and $\operatorname{imp}_{R} Z=0$.

(ii) $Z$ is Cohen-Macaulay.

Proof. The first inequality is a consequence of the New Intersection Theorem, cf. (3.6.1), while the second and third follow by (3.3) (a). The equivalence of conditions $(i)$ and $(i i)$ is evident from the inequalities. 
Closing this section we show that it is possible, for any semi-dualizing complex $C$, to define a $\mathrm{G}$-dimension that mimics the nice properties of the original. To be exact, (3.14) and (3.17) below are covered in the case $C=R$ by Theorem 2.9 and Lemmas 2.14 and 2.15 in [SYs95].

(3.11) Definition. Let $C$ be a semi-dualizing complex for $R$. For $Z \in \mathcal{D}_{\mathrm{b}}^{\mathrm{f}}(R)$ we define the $G$-dimension of $Z$ with respect to $C$ as follows:

$$
G-\operatorname{dim}_{C} Z= \begin{cases}\inf C-\inf Z^{\dagger_{C}} & \text { if } Z \in_{C} \mathcal{R}(R) ; \quad \text { and } \\ \infty & \text { if } Z \notin_{C} \mathcal{R}(R) .\end{cases}
$$

For $C=R$ this definition agrees with [SYs95 2.8].

(3.12) Lemma. Let $C$ be a semi-dualizing complex for $R$. If $Z \in \mathcal{D}_{\mathrm{b}}^{\mathrm{f}}(R)$, then

$$
\sup Z \leq \operatorname{amp} C+G-\operatorname{dim}_{C} Z ;
$$

and for $m \in \mathbb{Z}$ there are equalities:

$$
\begin{aligned}
G-\operatorname{dim}_{C} \Sigma^{m} Z & =G-\operatorname{dim}_{C} Z+m ; \quad \text { and } \\
G-\operatorname{dim}_{\Sigma^{m} C} Z & =G-\operatorname{dim}_{C} Z .
\end{aligned}
$$

Proof. First, note that the inequality as well as the two equalities trivially hold if $Z \notin{ }_{C} \mathcal{R}(R)$. For $Z \in{ }_{C} \mathcal{R}(R)$ the two equalities follow by inspection and the inequality follows by (1.3.3):

$$
\sup Z=\sup Z^{\dagger_{C} \dagger_{C}} \leq \sup C-\inf Z^{\dagger_{C}}=\operatorname{amp} C+G-\operatorname{dim}_{C} Z
$$

We can now compile some basic properties of the $\mathrm{G}$-dimension.

(3.13) Observation. Let $C$ be a semi-dualizing complex for $R$. The following hold for $Z \in \mathcal{D}_{\mathrm{b}}^{\mathrm{f}}(R)$ :

$$
\begin{aligned}
& G-\operatorname{dim}_{C} Z \in\{\infty\} \cup \mathbb{Z} \cup\{-\infty\} ; \\
& G-\operatorname{dim}_{C} Z \geq \sup Z-\operatorname{amp} C ; \\
& G-\operatorname{dim}_{C} Z=-\infty \quad \Longleftrightarrow \quad Z \simeq 0 ; \text { and } \\
& G-\operatorname{dim}_{C} Z<\infty \quad \Longleftrightarrow Z \in{ }_{C} \mathcal{R}(R) .
\end{aligned}
$$

The next theorem generalizes Auslander and Bridger's equality, $G-\operatorname{dim}_{R} M=$ $\operatorname{depth} R-\operatorname{depth}_{R} M$ [MAuMBr69, (4.13)(b)], for finite modules of finite Gorenstein dimension. This Auslander-Bridger Equality was, in turn, a generalization of the Auslander-Buchsbaum Equality for projective dimension, so it seems appropriate that an equality of this type should be called, simply, an ABE.

(3.14) Theorem (ABE for Reflexive Complexes). Let $C$ be a semi-dualizing complex for $R$. If $Z$ is $C$-reflexive, then

$$
G-\operatorname{dim}_{C} Z=\operatorname{depth} R-\operatorname{depth}_{R} Z .
$$

Proof. By (3.1) (a) and (3.2) (a) we have

$$
\begin{aligned}
G-\operatorname{dim}_{C} Z & =\inf C-\inf Z^{\dagger_{C}} \\
& =\inf C+\operatorname{depth}_{R} C-\operatorname{depth}_{R} Z \\
& =\operatorname{depth} R-\operatorname{depth}_{R} Z . \quad \square
\end{aligned}
$$


The next proposition shows that $\mathrm{G}$-dimension with respect to a semi-dualizing complex is a finer invariant than the projective dimension.

(3.15) Proposition. Let $C$ be a semi-dualizing complex for $R$. For $Z \in \mathcal{D}_{\mathrm{b}}^{\mathrm{f}}(R)$ there is an inequality:

$$
G-\operatorname{dim}_{C} Z \leq \operatorname{pd}_{R} Z
$$

and equality holds if $\operatorname{pd}_{R} Z<\infty$.

Proof. The inequality trivially holds if $\operatorname{pd}_{R} Z=\infty$. If $\operatorname{pd}_{R} Z<\infty$, then also $G$ - $\operatorname{dim}_{C} Z$ is finite, cf. (2.9), and we have $\operatorname{pd}_{R} Z=\operatorname{depth} R-\operatorname{depth}_{R} Z$ by the Auslander-Buchsbaum Equality, cf. [HBF80, (0.1)], so equality holds by (3.14).

Let $C$ be a semi-dualizing complex for $R$, and let $\mathfrak{p} \in \operatorname{Spec} R$. For $Z \in \mathcal{P}^{\mathrm{f}}(R)$ there is an inequality $G$ - $\operatorname{dim}_{C_{\mathfrak{p}}} Z_{\mathfrak{p}} \leq G$ - $\operatorname{dim}_{C} Z$, as the $\mathrm{G}$-dimension equals the projective dimension. However, such an inequality need not hold in general for $Z \in{ }_{C} \mathcal{R}(R)$; for example, we have $G-\operatorname{dim}_{C_{\mathfrak{p}}} C_{\mathfrak{p}}=\inf C_{\mathfrak{p}} \geq \inf C=G-\operatorname{dim}_{C} C$. If $C$ is a semi-dualizing module (that is, $\operatorname{amp} C=0$ ), then the desired inequality holds for all $C$-reflexive complexes; but in general, the best we will get is the following:

(3.16) Proposition. Let $C$ be a semi-dualizing complex for $R$. For $Z \in \mathcal{D}_{\mathrm{b}}^{\mathrm{f}}(R)$ and $\mathfrak{p} \in \operatorname{Spec} R$ there is an inequality:

$$
G-\operatorname{dim}_{C_{\mathfrak{p}}} Z_{\mathfrak{p}} \leq G-\operatorname{dim}_{C} Z+\inf C_{\mathfrak{p}}-\inf C .
$$

Proof. Suppose $G-\operatorname{dim}_{C} Z<\infty$, i.e., $Z \in{ }_{C} \mathcal{R}(R)$, then $Z_{\mathfrak{p}} \in C_{\mathfrak{p}} \mathcal{R}(R)$ by (2.10), and a straightforward computation establishes the desired inequality:

$$
\begin{aligned}
G-\operatorname{dim}_{C_{\mathfrak{p}}} Z_{\mathfrak{p}} & =\inf C_{\mathfrak{p}}-\inf \left(Z^{\dagger_{C}}\right)_{\mathfrak{p}} \\
& \leq \inf C_{\mathfrak{p}}-\inf Z^{\dagger_{C}}=G-\operatorname{dim}_{C} Z+\inf C_{\mathfrak{p}}-\inf C
\end{aligned}
$$

The behavior of $\mathrm{G}$-dimension under completion is accounted for in (5.11).

Auslander categories have some remarkable stability properties. For the Auslander and Bass classes (to be introduced in the next section) these are discussed in $\left[\mathrm{HBF}\right.$; here we only deal with those pertaining to the class ${ }_{C} \mathcal{R}(R)$ of reflexive complexes.

(3.17) Theorem. Let $C$ be a semi-dualizing complex for $R$. If $Z \in{ }_{C} \mathcal{R}(R)$ and $P \in \mathcal{P}^{\mathrm{f}}(R)$, then also the complexes $\operatorname{RHom}_{R}(P, Z)$ and $P \otimes_{R}^{\mathbf{L}} Z$ belong to ${ }_{C} \mathcal{R}(R)$, and there are equalities:

(a) $G-\operatorname{dim}_{C} \mathbf{R} \operatorname{Hom}_{R}(P, Z)=G-\operatorname{dim}_{C} Z-\inf P$; and

(b) $G-\operatorname{dim}_{C}\left(P \otimes_{R}^{\mathbf{L}} Z\right)=G-\operatorname{dim}_{C} Z+\operatorname{pd}_{R} P$.

Proof. Let $Z \in{ }_{C} \mathcal{R}(R)$ and $P \in \mathcal{P}^{\mathrm{f}}(R)$. The $\operatorname{complexes} \operatorname{RHom}_{R}(P, Z)$ and $P \otimes_{R}^{\mathbf{L}} Z$ belong to $\mathcal{D}_{\mathrm{b}}^{\mathrm{f}}(R)$, cf. (1.4), and so do their dagger duals as we have

$$
\begin{aligned}
& \left(P \otimes_{R}^{\mathbf{L}} Z\right)^{\dagger_{C}} \simeq \mathbf{R H o m}_{R}\left(P, Z^{\dagger_{C}}\right) \text { by (1.5.2); and } \\
& \mathbf{R H o m}_{R}(P, Z)^{\dagger_{C}} \simeq P \otimes_{R}^{\mathbf{L}} Z^{\dagger_{C}} \quad \text { by (1.5.8). }
\end{aligned}
$$


Moreover, the commutative diagrams below show that the relevant biduality maps are invertible.

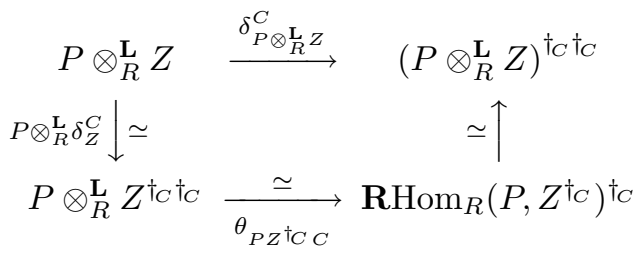

The evaluation morphisms $\theta_{P Z^{\dagger} C}$ and $\theta_{P Z C}$ are invertible as $P \in \mathcal{P}^{\mathrm{f}}(R)$, cf. (1.5.8).

$$
\begin{aligned}
& \mathbf{R H o m}_{R}(P, Z) \stackrel{\delta_{\mathbf{R H o m}}^{C}(P, Z)}{\longrightarrow} \mathbf{R H o m}_{R}(P, Z)^{\dagger_{C}^{C} \dagger_{C}} \\
& \mathbf{R H o m}_{R}\left(P, \delta_{Z}^{C}\right) \downarrow \simeq \quad \simeq \downarrow\left(\theta_{P Z C}\right)^{\dagger} \\
& \mathbf{R H o m}_{R}\left(P, Z^{\dagger_{C} \dagger_{C}}\right) \stackrel{\simeq}{\longrightarrow}\left(P \otimes_{R}^{\mathbf{L}} \mathbf{R} \operatorname{Hom}_{R}(Z, C)\right)^{\dagger_{C}}
\end{aligned}
$$

This proves that $\mathbf{R H o m}_{R}(P, Z)$ and $P \otimes_{R}^{\mathbf{L}} Z$ belong to ${ }_{C} \mathcal{R}(R)$, i.e., they have finite $\mathrm{G}$-dimension, and the next step is to compute these dimensions.

(a): The desired equality follows by the definition of $\mathrm{G}$-dimension and the next computation:

$$
\begin{aligned}
& -\inf \left(\mathbf{R H o m}_{R}(P, Z)^{\dagger_{C}}\right)=-\inf \left(P \otimes_{R}^{\mathbf{L}} Z^{\dagger_{C}}\right) \text { by (1.5.8) } \\
& =-\inf Z^{\dagger_{C}}-\inf P \text { by (1.3.4). }
\end{aligned}
$$

(b): We have $\left(P \otimes{ }_{R}^{\mathbf{L}} Z\right)^{\dagger_{C}} \simeq \mathbf{R} \operatorname{Hom}_{R}\left(P, Z^{\dagger_{C}}\right)$ by (1.5.2), and hence

$$
\begin{aligned}
-\inf \left(P \otimes_{R}^{\mathbf{L}} Z\right)^{\dagger_{C}} & =-\operatorname{ord} \mathrm{P}_{\mathbf{R} \operatorname{Hom}_{R}\left(P, Z^{\dagger^{\dagger}}\right)}^{R}(t) \\
& =-\operatorname{ord}\left(\mathrm{P}_{P}^{R}\left(t^{-1}\right) \mathrm{P}_{Z^{\dagger}}^{R}(t)\right) \quad \text { by (2.14) } \\
& =\operatorname{deg} \mathrm{P}_{P}^{R}(t)-\operatorname{ord} \mathrm{P}_{Z^{\dagger^{\dagger} C}}^{R}(t) \\
& =\operatorname{pd}_{R} P-\inf Z^{\dagger_{C}} .
\end{aligned}
$$

Again the desired equality follows by the definition of G-dimension.

(3.18) Observation. Let $C$ be a semi-dualizing complex for $R$. If $Z$ is $C$-reflexive, then $Z \simeq \mathbf{R H o m}_{R}\left(Z^{t_{c}}, C\right)$, so by (1.7.7) there is an equality of formal Laurant series:

$$
\mathrm{I}_{R}^{Z}(t)=\mathrm{P}_{Z^{\dagger} C}^{R}(t) \mathrm{I}_{R}^{C}(t)
$$

In particular,

$$
\mathrm{I}_{R}(t)=\mathrm{P}_{C}^{R}(t) \mathrm{I}_{R}^{C}(t) .
$$

If $D$ is dualizing for $R$, then $\mathrm{I}_{R}^{D}(t)=t^{d}$ for some $d \in \mathbb{Z}$, cf. (1.8.1), and for every complex $Z$ in $\mathcal{D}_{\mathrm{b}}^{\mathrm{f}}(R)$ we have

$$
\mathrm{I}_{R}^{Z}(t)=\mathrm{P}_{Z^{\dagger} D}^{R}(t) t^{d} \quad \text { and } \quad \mathrm{P}_{Z}^{R}(t)=\mathrm{I}_{R}^{Z^{\dagger} D}(t) t^{-d} .
$$

Proof of (3.1). (a): By (1.7.1), (3.18.1), and (1.7.3) we have

$$
\operatorname{depth}_{R} Z=\operatorname{ord} \mathrm{I}_{R}^{Z}(t)=\operatorname{ord} \mathrm{P}_{Z^{\dagger_{C}}}^{R}(t)+\operatorname{ord} \mathrm{I}_{R}^{C}(t)=\inf Z^{\dagger_{C}}+\operatorname{depth}_{R} C .
$$

(d): The equality is evident by the next chain of inclusions:

$$
\operatorname{Supp}_{R} Z=\operatorname{Supp}_{R} Z^{\dagger_{C} \dagger_{C}} \subseteq \operatorname{Supp}_{R} Z^{\dagger_{C}} \subseteq \operatorname{Supp}_{R} Z \text {. }
$$


(b): Using (d) and applying (a) to $\left(Z^{\dagger_{C}}\right)_{\mathfrak{p}} \in{C_{\mathfrak{p}}}_{\mathcal{R}} \mathcal{R}\left(R_{\mathfrak{p}}\right)$, cf. (2.10), we find that

$$
\begin{aligned}
\operatorname{dim}_{R} Z & =\sup \left\{\operatorname{dim} R / \mathfrak{p}-\inf Z_{\mathfrak{p}} \mid \mathfrak{p} \in \operatorname{Supp}_{R} Z\right\} \\
& =\sup \left\{\operatorname{dim} R / \mathfrak{p}+\operatorname{depth}_{R_{\mathfrak{p}}} C_{\mathfrak{p}}-\operatorname{depth}_{R_{\mathfrak{p}}}\left(Z^{\dagger_{C}}\right)_{\mathfrak{p}} \mid \mathfrak{p} \in \operatorname{Supp}_{R} Z^{\dagger_{C}}\right\} .
\end{aligned}
$$

Thus, it follows by (1.7.5), 1.6.4), and (1.6.6) that

$$
\begin{aligned}
\operatorname{dim}_{R} Z & \geq \sup \left\{\operatorname{depth}_{R} C-\operatorname{depth}_{R_{\mathfrak{p}}}\left(Z^{\dagger_{C}}\right)_{\mathfrak{p}} \mid \mathfrak{p} \in \operatorname{Supp}_{R} Z^{\dagger_{C}}\right\} \\
& =\operatorname{depth}_{R} C+\sup Z^{\dagger_{C}} ;
\end{aligned}
$$

and by (1.6.7) and (1.6.5) we get

$$
\begin{aligned}
\operatorname{dim}_{R} Z & \leq \sup \left\{\operatorname{dim} R / \mathfrak{p}+\operatorname{dim}_{R_{\mathfrak{p}}} C_{\mathfrak{p}}-\operatorname{depth}_{R_{\mathfrak{p}}}\left(Z^{\dagger_{C}}\right)_{\mathfrak{p}} \mid \mathfrak{p} \in \operatorname{Supp}_{R} Z^{\dagger_{C}}\right\} \\
& \leq \sup \left\{\operatorname{dim}_{R} C-\operatorname{depth}_{R_{\mathfrak{p}}}\left(Z^{\dagger_{C}}\right)_{\mathfrak{p}} \mid \mathfrak{p} \in \operatorname{Supp}_{R} Z^{\dagger_{C}}\right\} \\
& =\operatorname{dim}_{R} C+\sup Z^{\dagger_{C}} .
\end{aligned}
$$

(c): Again we use (d), and the desired inequality follows by a straightforward computation:

$$
\begin{aligned}
\operatorname{dim}_{R} Z-\sup Z^{\dagger_{C}} & =\sup \left\{\operatorname{dim} R / \mathfrak{p}-\inf Z_{\mathfrak{p}}-\sup Z^{\dagger_{C}} \mid \mathfrak{p} \in \operatorname{Supp}_{R} Z\right\} \\
& \leq \sup \left\{\operatorname{dim} R / \mathfrak{p}-\inf Z_{\mathfrak{p}}-\inf \left(Z^{\dagger_{C}}\right)_{\mathfrak{p}} \mid \mathfrak{p} \in \operatorname{Supp}_{R} Z^{\dagger_{C}}\right\} \\
& \leq \sup \left\{\operatorname{dim} R / \mathfrak{p}-\inf \left(Z^{\dagger_{C}}\right)_{\mathfrak{p}}-\inf Z \mid \mathfrak{p} \in \operatorname{Supp}_{R} Z^{\dagger_{C}}\right\} \\
& =\operatorname{dim}_{R} Z^{\dagger_{C}}-\inf Z .
\end{aligned}
$$

\section{Foxby Duality}

When $C$ is semi-dualizing for $R$, canonical maps $\gamma_{X}^{C}: X \rightarrow \mathbf{R H o m}_{R}\left(C, C \otimes \otimes_{R}^{\mathbf{L}} X\right)$ and $\xi_{Y}^{C}: C \otimes_{R}^{\mathbf{L}} \mathbf{R H o m}_{R}(C, Y) \rightarrow Y$, for $X, Y \in \mathcal{D}_{\mathrm{b}}(R)$, are defined by requiring commutativity of the diagrams

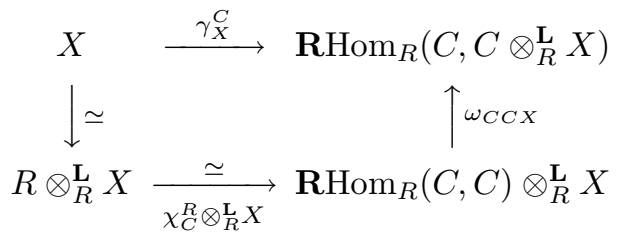

and

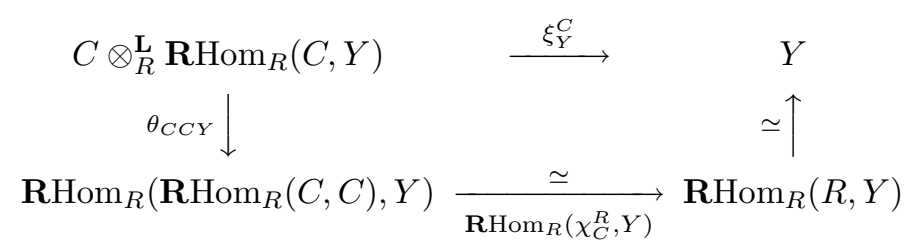

(4.1) Definitions. Let $C$ be a semi-dualizing complex for $R$. The $C$-Auslander class of $R,{ }_{C} \mathcal{A}(R)$, and the $C$-Bass class of $R,{ }_{C} \mathcal{B}(R)$, are the full subcategories of $\mathcal{D}_{\mathrm{b}}(R)$ defined by specifying their objects as follows:

(A) $X$ belongs to ${ }_{C} \mathcal{A}(R)$ if and only if $C \otimes_{R}^{\mathbf{L}} X \in \mathcal{D}_{\mathrm{b}}(R)$ and the canonical map $\gamma_{X}^{C}: X \rightarrow \mathbf{R H o m}_{R}\left(C, C \otimes_{R}^{\mathbf{L}} X\right)$ is an isomorphism.

(B) $Y$ belongs to ${ }_{C} \mathcal{B}(R)$ if and only if $\mathbf{R H o m}_{R}(C, Y) \in \mathcal{D}_{\mathrm{b}}(R)$ and the canonical $\operatorname{map} \xi_{Y}^{C}: C \otimes \mathbb{L}_{R}^{\mathbf{L}} \mathbf{R H o m}_{R}(C, Y) \rightarrow Y$ is an isomorphism. 
(4.2) Genuine Foxby Duality. Let $R$ be a local ring. If $D$ is a dualizing complex for $R$, then there is a commutative diagram

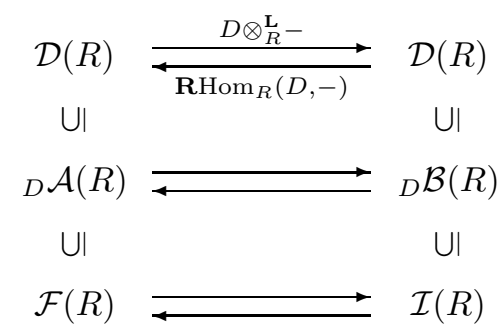

where the vertical inclusions are full embeddings, and the unlabeled horizontal arrows are quasi-inverse equivalences of categories. This has become known as Foxby duality, cf. [EJX96] and [JXu96]; a proof is given in [LLAHBF97, (3.2)].

The purpose of this section is to establish a theory of Foxby duality for semidualizing complexes. We seek to take the analogy with (4.2) as far as possible, and it does go quite far: the full embeddings are established in (4.4) and the equivalence of Auslander and Bass classes in (4.6). On the other hand, it is out of the question that every semi-dualizing complex $C$ will give equivalences of $\mathcal{F}(R)$ and $\mathcal{I}(R)$ the way a dualizing complex does. For $C=R$, e.g., this would imply that $\mathcal{F}(R)=\mathcal{I}(R)$, which means that $R$ is Gorenstein. This problem is treated carefully in section 8 ,

(4.3) Remarks. It is straightforward to check that $R \in{ }_{C} \mathcal{A}(R)$ and $C \in{ }_{C} \mathcal{B}(R)$ when $C$ is a semi-dualizing complex for $R$; and, obviously, ${ }_{R} \mathcal{A}(R)={ }_{R} \mathcal{B}(R)=$ $\mathcal{D}_{\mathrm{b}}(R)$. When $R$ is local and Cohen-Macaulay with a dualizing module $D$, the complexes in the Auslander class ${ }_{D} \mathcal{A}(R)$ are exactly those of finite Gorenstein projective dimension or, equivalently, finite Gorenstein flat dimension, while the complexes in the Bass class ${ }_{D} \mathcal{B}(R)$ are those of finite Gorenstein injective dimension, cf. LLAHBF97, EJX96, [HBF], and [LWC0.

Auslander and Bass classes behave as expected under completion and localization. That is, $X_{\mathfrak{p}} \in{ }_{C_{\mathfrak{p}}} \mathcal{A}\left(R_{\mathfrak{p}}\right)$ if $X \in{ }_{C} \mathcal{A}(R)$; and if $R$ is local, then $X$ belongs to ${ }_{C} \mathcal{A}(R)$ if and only if $X \otimes_{R} \widehat{R} \in{ }_{C \otimes_{R} \widehat{R}} \mathcal{A}(\widehat{R})$. Similar results hold for Bass classes; they are all special cases of (5.8) and (5.9).

(4.4) Proposition. If $C$ is a semi-dualizing complex for $R$, then there are two full embeddings:

$$
\mathcal{F}(R) \subseteq{ }_{C} \mathcal{A}(R) \quad \text { and } \quad \mathcal{I}(R) \subseteq{ }_{C} \mathcal{B}(R)
$$

Proof. The first embedding is immediate by (1.4.1) and (4.0.1) and the second by (1.4.2) and 4.0.2).

(4.5) Lemma. Let $C$ be a semi-dualizing complex for $R$. The following hold for $X \in{ }_{C} \mathcal{A}(R), Y \in{ }_{C} \mathcal{B}(R)$, and $U \in \mathcal{D}_{\mathrm{b}}(R)$ :

$$
\mathbf{R H o m}_{R}(U, X) \simeq \mathbf{R H o m}_{R}\left(C \otimes_{R}^{\mathbf{L}} U, C \otimes_{R}^{\mathbf{L}} X\right) ;
$$




$$
\mathbf{R H o m}_{R}(Y, U) \simeq \mathbf{R} \operatorname{Hom}_{R}\left(\mathbf{R} \operatorname{Hom}_{R}(C, Y), \mathbf{R H o m}_{R}(C, U)\right) ;
$$

and

$$
Y \otimes_{R}^{\mathbf{L}} U \simeq\left(C \otimes_{R}^{\mathbf{L}} U\right) \otimes_{R}^{\mathbf{L}} \mathbf{R} \operatorname{Hom}_{R}(C, Y) .
$$

Proof. The proof of (a) is straightforward:

$$
\begin{aligned}
\operatorname{RHom}_{R}(U, X) & \simeq \mathbf{R H o m}_{R}\left(U, \mathbf{R H o m}_{R}\left(C, C \otimes_{R}^{\mathbf{L}} X\right)\right) \\
& \simeq \mathbf{R} \operatorname{Hom}_{R}\left(U \otimes_{R}^{\mathbf{L}} C, C \otimes_{R}^{\mathbf{L}} X\right) \\
& \simeq \mathbf{R H o m}_{R}\left(C \otimes_{R}^{\mathbf{L}} U, C \otimes_{R}^{\mathrm{L}} X\right) ;
\end{aligned}
$$

and the proofs of (b) and (c) are similar.

(4.6) Foxby Duality Theorem. Let $C$ be a semi-dualizing complex for $R$. The two functors $C \otimes{ }_{R}^{\mathbf{L}}-$ and $\mathbf{R H o m}_{R}(C,-)$ give quasi-inverse equivalences of ${ }_{C} \mathcal{A}(R)$ and ${ }_{C} \mathcal{B}(R)$, and they take semi-dualizing complex es to semi-dualizing complexes.

Furthermore, the following hold for $X, Y \in \mathcal{D}_{\mathrm{b}}(R)$ :

(a) If $C \otimes{ }_{R}^{\mathrm{L}} X \in{ }_{C} \mathcal{B}(R)$, then $X \in{ }_{C} \mathcal{A}(R)$; and

(b) if $\mathbf{R H o m}_{R}(C, Y) \in{ }_{C} \mathcal{A}(R)$, then $Y \in{ }_{C} \mathcal{B}(R)$.

Proof. The proof of [LLAHBF97. (3.2)] applies verbatim to show that $C \otimes_{R}^{\mathbf{L}}-$ and $\mathbf{R H o m}_{R}(C,-)$ give quasi-inverse equivalences of ${ }_{C} \mathcal{A}(R)$ and ${ }_{C} \mathcal{B}(R)$ with properties (a) and (b).

Suppose $X \in{ }_{C} \mathcal{A}(R)$ is semi-dualizing for $R$, then $C \otimes_{R}^{\mathrm{L}} X \in \mathcal{D}_{\mathrm{b}}^{\mathrm{f}}(R)$, cf. (1.3.1), and we have

$$
R \stackrel{\chi_{X}^{R}}{\simeq} \mathbf{R H o m}_{R}(X, X) \simeq \mathbf{R H o m}_{R}\left(C \otimes_{R}^{\mathbf{L}} X, C \otimes_{R}^{\mathbf{L}} X\right) .
$$

The last isomorphism is (4.5) (a), it only involves natural maps, and it is straightforward to check that also $\chi_{C \otimes{ }_{R} X}^{\mathrm{L} X}$ is an isomorphism. A similar argument, using (4.5) (b), shows that $\mathbf{R H o m}_{R}(C, Y)$ is semi-dualizing for $R$ if $Y \in{ }_{C} \mathcal{B}(R)$ is so.

The next result provides a connection to the concept of (generalized) G-dimension introduced in previous section. As a corollary we recover [LLAHBF97, (4.1.7)], the fact that: if $R$ is local with a dualizing complex $D$, then the finite modules in ${ }_{D} \mathcal{A}(R)$ are exactly those with $G-\operatorname{dim}_{R} M<\infty$.

(4.7) Theorem. Let $R$ be local, and let $C$ be a semi-dualizing complex for $R$. If $D$ is dualizing for $R$, then there is an equality of full subcategories:

$$
{ }_{C} \mathcal{A}^{\mathrm{f}}(R)={ }_{C^{\dagger_{D}}} \mathcal{R}(R) .
$$

Proof. Set $B=C^{\dagger_{D}}, B$ is semi-dualizing for $R$ by (2.12), and we want to prove that ${ }_{C} \mathcal{A}^{\mathrm{f}}(R)={ }_{B} \mathcal{R}(R)$. 
First, note that for $Z \in \mathcal{D}_{\mathrm{b}}^{\mathrm{f}}(R)$ we have two chains of isomorphisms:

$$
\begin{aligned}
Z^{\dagger_{B}} & =\mathbf{R H o m}_{R}\left(Z, \mathbf{R H o m}_{R}(C, D)\right) \\
& \simeq \mathbf{R} \operatorname{Hom}_{R}\left(Z \otimes_{R}^{\mathbf{L}} C, D\right) \\
& \simeq\left(C \otimes_{R}^{\mathbf{L}} Z\right)^{\dagger_{D}} \\
& \simeq \mathbf{R} H_{R}\left(C, Z^{\dagger_{D}}\right) ; \text { and } \\
C \otimes_{R}^{\mathbf{L}} Z & \simeq C^{\dagger_{D} \dagger_{D}} \otimes_{R}^{\mathbf{L}} Z \\
& \simeq Z \otimes_{R}^{\mathbf{L}} \mathbf{R} \operatorname{Hom}_{R}(B, D) \\
& \simeq \mathbf{R} \operatorname{Hom}_{R}\left(\mathbf{R H o m}_{R}(Z, B), D\right) \text { by } \mathbf{1 . 5 . 8} \\
& =\left(Z^{\dagger_{B}}\right)^{\dagger_{D}} .
\end{aligned}
$$

Thus, if $Z$ and $Z^{\dagger_{B}}$ belong to $\mathcal{D}_{\mathrm{b}}^{\mathrm{f}}(R)$ we have a commutative diagram

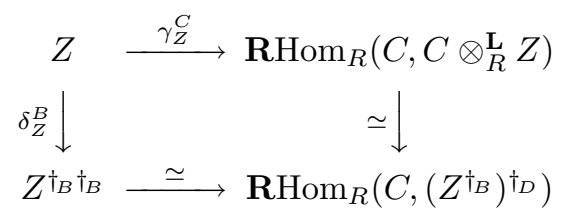

where the unlabeled maps are (induced by) $(*)$ and $(* *)$.

" $\subseteq$ ": Suppose $Z \in{ }_{C} \mathcal{A}^{\mathrm{f}}(R)$, then $C \otimes_{R}^{\mathbf{L}} Z$, and thereby $\left(C \otimes_{R}^{\mathbf{L}} Z\right)^{\dagger_{D}}$, belongs to $\mathcal{D}_{\mathrm{b}}^{\mathrm{f}}(R)$, cf. (1.4.2). By $(*)$ we conclude that $Z^{\dagger_{B}} \in \mathcal{D}_{\mathrm{b}}^{\mathrm{f}}(R)$. The canonical map $\gamma_{Z}^{C}$ is an isomorphism, and the diagram above shows that so is $\delta_{Z}^{B}$.

"D": Let $Z \in{ }_{B} \mathcal{R}(R)$, then $Z^{\dagger_{B}} \in \mathcal{D}_{\mathrm{b}}^{\mathrm{f}}(R)$ and hence also $\left(Z^{\dagger_{B}}\right)^{\dagger_{D}} \in \mathcal{D}_{\mathrm{b}}^{\mathrm{f}}(R)$, so (**) shows that $C \otimes_{R}^{\mathrm{L}} Z \in \mathcal{D}_{\mathrm{b}}^{\mathrm{f}}(R)$. The biduality map $\delta_{Z}^{B}$ is an isomorphism, and the diagram shows that so is $\gamma_{Z}^{C}$.

The last results of this section answer the question of 'how much the size of a complex can change under Foxby duality', and it paves the way for a description of Foxby duality for semi-dualizing modules in terms of classical homological algebra. The proof of the next inequalities is found at the end of the section.

(4.8) Proposition. Let $C$ be a semi-dualizing complex for $R$. For $X \in{ }_{C} \mathcal{A}(R)$ and $Y \in{ }_{C} \mathcal{B}(R)$ there are inequalities:

$$
\begin{aligned}
\sup X+\inf C & \leq \sup \left(C \otimes_{R}^{\mathrm{L}} X\right) \leq \sup X+\sup C ; \\
\operatorname{amp} X-\operatorname{amp} C & \leq \operatorname{amp}\left(C \otimes_{R}^{\mathrm{L}} X\right) \leq \operatorname{amp} X+\operatorname{amp} C ; \\
\inf Y-\sup C & \leq \inf \left(\mathbf{R H o m} H_{R}(C, Y)\right) \leq \inf Y-\inf C ; \quad \text { and } \\
\operatorname{amp} Y-\operatorname{amp} C & \leq \operatorname{amp}\left(\mathbf{R} \operatorname{Hom}_{R}(C, Y)\right) \leq \operatorname{amp} Y+\operatorname{amp} C .
\end{aligned}
$$

(4.9) Corollary. Let $C$ be a semi-dualizing complex for $R$. The next three conditions are equivalent.

(i) $\operatorname{amp} C=0$.

(ii) $\operatorname{amp}\left(C \otimes_{R}^{\mathrm{L}} X\right)=\operatorname{amp} X$ for all $X \in{ }_{C} \mathcal{A}(R)$.

(iii) $\operatorname{amp}\left(\mathbf{R} \operatorname{Hom}_{R}(C, Y)\right)=\operatorname{amp} Y$ for all $Y \in{ }_{C} \mathcal{B}(R)$.

And if $R$ is local and $D$ is dualizing for $R$, then they are equivalent with: 
(iv) $C^{\dagger_{D}}$ is Cohen-Macaulay.

Proof. The implications $(i) \Rightarrow(i i)$ and $(i) \Rightarrow($ iii $)$ follow by (b) and (d) in (4.8), and the reverse implications follow by setting $X=R$ and $Y=C$. If $R$ is local and $D$ is dualizing for $R$, then $\operatorname{cmd}_{R} C^{\dagger_{D}}=\operatorname{amp} C^{\dagger_{D} \dagger_{D}}=\operatorname{amp} C$, cf. (3.8), and hence $(i v)$ is equivalent to $(i)$.

(4.10) Observation. Corollary (4.9) generalizes [LLAHBF97, (3.3)]. A semi-dualizing complex $C$ satisfying the equivalent conditions in (4.9) has only one nonvanishing homology module, and we may, after a shift, assume that it is located in degree 0 and hence identify $C$ with the module $K=\mathrm{H}_{0}(C)$. Thus, $K$ is a semidualizing module: The endomorphism $\operatorname{ring} \operatorname{Hom}_{R}(K, K)$ is canonically isomorphic to $R$, and $\operatorname{Ext}_{R}^{i}(K, K)=0$ for $i>0$.

Modules in the Auslander and Bass classes ${ }_{K} \mathcal{A}(R)$ and ${ }_{K} \mathcal{B}(R)$ can be easily described in terms of classical homological algebra:

$$
M \in{ }_{K} \mathcal{A}_{0}(R) \Longleftrightarrow \begin{cases}\operatorname{Tor}_{i}^{R}(K, M)=0 & \text { for } i>0 ; \\ \operatorname{Ext}_{R}^{i}\left(K, K \otimes_{R} M\right)=0 & \text { for } i>0 ; \text { and } \\ M \cong \operatorname{Hom}_{R}\left(K, K \otimes_{R} M\right) & \text { canonically. }\end{cases}
$$

Similarly, $N$ belongs to ${ }_{K} \mathcal{B}_{0}(R)$ if and only if

$$
\operatorname{Ext}_{R}^{i}(K, N)=0=\operatorname{Tor}_{i}^{R}\left(K, \operatorname{Hom}_{R}(K, N)\right)
$$

for $i>0$ and $K \otimes_{R} \operatorname{Hom}_{R}(K, N)$ is canonically isomorphic to $N$. The functors $K \otimes_{R}-$ and $\operatorname{Hom}_{R}(K,-)$ give quasi-inverse equivalences of the categories ${ }_{K} \mathcal{A}_{0}(R)$ and ${ }_{K} \mathcal{B}_{0}(R)$. These claims are proved in detail in [LLAHBF97, (3.4) and (3.6)] for the case where $K$ is dualizing (and $R$ hence local Cohen-Macaulay), and it is again easily verified that the proofs apply verbatim in our setting. Semi-dualizing modules are $P G$-modules (of rank 1) and partial proofs can be found in [HBF72].

(4.11) Lemma. Let $C$ be a semi-dualizing complex for $R$. For $X \in \mathcal{D}_{\mathrm{b}}(R)$ the next two inequalities hold.

$$
\begin{aligned}
\sup X-\sup C & \leq \sup \left(\mathbf{R} \operatorname{Hom}_{R}(C, X)\right) \leq \sup X-\inf C ; \quad \text { and } \\
\inf X+\inf C & \leq \inf \left(C \otimes_{R}^{\mathbf{L}} X\right) \leq \inf X+\sup C .
\end{aligned}
$$

Proof. The first inequality in (a) follows by [HBF77, 2.2] as $\operatorname{Supp}_{R} C=\operatorname{Spec} R$, cf. (2.5), and the second is (1.3.3).

Let $J$ be a faithfully injective $R$-module (e.g., $J=\prod_{\mathfrak{m} \in \operatorname{Max} R} \mathrm{E}_{R}(R / \mathfrak{m})$, where $\mathrm{E}_{R}(M)$ denotes the injective hull of an $R$-module $M$ ); by (a) we have

$$
\begin{aligned}
\inf \left(C \otimes_{R}^{\mathbf{L}} X\right) & =-\sup \left(\operatorname{Hom}_{R}\left(C \otimes_{R}^{\mathbf{L}} X, J\right)\right) \\
& =-\sup \left(\mathbf{R} \operatorname{Hom}_{R}\left(C, \operatorname{Hom}_{R}(X, J)\right)\right) \\
& \leq \sup C-\sup \left(\operatorname{Hom}_{R}(X, J)\right) \\
& =\sup C+\inf X .
\end{aligned}
$$

This proves the second inequality in (b), and the first one is (1.3.4).

Proof of (4.8). (a): Since $X \simeq \mathbf{R H o m}_{R}\left(C, C \otimes \otimes_{R}^{\mathbf{L}} X\right)$ it follows by (4.11) (a) that

$$
\sup \left(C \otimes_{R}^{\mathbf{L}} X\right)-\sup C \leq \sup X \leq \sup \left(C \otimes_{R}^{\mathbf{L}} X\right)-\inf C
$$


and, therefore,

$$
-\sup X-\sup C \leq-\sup \left(C \otimes_{R}^{\mathrm{L}} X\right) \leq-\sup X-\inf C .
$$

(b): Using the inequalities in (a) and (4.11) (b) we find:

$$
\begin{aligned}
\operatorname{amp}\left(C \otimes_{R}^{\mathbf{L}} X\right) & =\sup \left(C \otimes_{R}^{\mathbf{L}} X\right)-\inf \left(C \otimes_{R}^{\mathbf{L}} X\right) \\
& \leq \sup X+\sup C-\inf \left(C \otimes_{R}^{\mathbf{L}} X\right) \\
& \leq \sup X+\sup C-(\inf X+\inf C) \\
& =\operatorname{amp} X+\operatorname{amp} C ; \text { and } \\
\operatorname{amp}\left(C \otimes_{R}^{\mathbf{L}} X\right) & =\sup \left(C \otimes_{R}^{\mathbf{L}} X\right)-\inf \left(C \otimes_{R}^{\mathbf{L}} X\right) \\
& \geq \sup X+\inf C-\inf \left(C \otimes_{R}^{\mathbf{L}} X\right) \\
& \geq \sup X+\inf C-(\inf X+\sup C) \\
& =\operatorname{amp} X-\operatorname{amp} C .
\end{aligned}
$$

The proof of (c) is similar to that of (a), only it uses (4.11)(b). The proof of (d) uses (c) and 4.11) (a), otherwise it is analogous to the proof of (b).

\section{Base Change}

In this section $\varphi: R \rightarrow S$ is a homomorphism of rings.

We study the behavior of semi-dualizing complexes and Auslander categories under base change; the focus is on (local) homomorphisms of finite flat dimension.

The proofs of the principal results (5.1), (5.3), (5.8), (5.9), and (5.10) require some technical auxiliary results, and they have, therefore, been gathered at the end of the section.

(5.1) Theorem. Let $C$ be a semi-dualizing complex for $R$. The next two conditions are equivalent.

(i) $S \in{ }_{C} \mathcal{A}(R)$.

(ii) $C \otimes_{R}^{\mathbf{L}} S$ is semi-dualizing for $S$.

When they are satisfied, there is an inequality of amplitudes:

$$
\operatorname{amp}\left(C \otimes_{R}^{\mathrm{L}} S\right) \leq \operatorname{amp} C ;
$$

and, provided that $\varphi$ is local, an equality of formal Laurant series:

$$
\mathrm{I}_{S}(t)=\mathrm{P}_{C}^{R}(t) \mathrm{I}_{S}^{C \otimes_{R}^{\mathrm{L}} S}(t) .
$$

Furthermore, if $S$ is local and $E$ is a dualizing complex for $S$, then

$$
\left(C \otimes_{R}^{\mathbf{L}} S\right)^{\dagger_{E}} \simeq \mathbf{R H o m}_{R}(C, E),
$$

and the next two conditions are equivalent and equivalent to $(i)$ and (ii).

(iii) $E \in{ }_{C} \mathcal{B}(R)$.

(iv) $\mathbf{R H o m}_{R}(C, E)$ is semi-dualizing for $S$.

(5.2) Remarks. Theorem (5.1) is is the general result on base change for semidualizing complexes, special cases will be considered below; among its consequences we note the following: 
Applying it to a dualizing complex $C$ for $R$ we obtain a converse to the key result in LLAHBF97 on existence of relative dualizing complexes, see also (7.1).

Suppose $\varphi$ is local and $S$ belongs to ${ }_{C} \mathcal{A}(R)$; the equality (5.1) (b) generalizes LLAHBF97, (7.1)] and shows that if $S$ is Gorenstein, then $\mathrm{P}_{C}^{R}(t)$ is a monomial and hence $C \sim R$ as will be proved in (8.3); this should be compared to [LLAHBF97. (7.7.2)].

The following descent result generalizes [LLAHBF97, (4.6)(a) and (7.9)].

(5.3) Proposition. Let $C$ be a semi-dualizing complex for $R$, and assume that $S \in{ }_{C} \mathcal{A}(R)$. The base changed complex $C \otimes_{R}^{\mathbf{L}} S$ is then semi-dualizing for $S$, and the following hold for $S$-complexes $X$ and $Y$ :

$$
\begin{aligned}
X \in_{C \otimes_{R}^{\mathrm{L}} S} \mathcal{A}(S) & \Longleftrightarrow X \in_{C} \mathcal{A}(R) ; \quad \text { and } \\
Y \in_{C \otimes_{R}^{\mathrm{L}} S} \mathcal{B}(S) & \Longleftrightarrow Y \in_{C} \mathcal{B}(R) .
\end{aligned}
$$

In particular, there are full embeddings:

$$
\begin{aligned}
& \mathcal{F}(S) \subseteq{ }_{C \otimes_{R}{ }_{R} S} \mathcal{A}(S) \subseteq{ }_{C} \mathcal{A}(R) ; \quad \text { and } \\
& \mathcal{I}(S) \subseteq{ }_{C \otimes_{R}^{\mathrm{L}} S} \mathcal{B}(S) \subseteq{ }_{C} \mathcal{B}(R) .
\end{aligned}
$$

We now turn our attention to homomorphisms of finite flat dimension; we start by reviewing a few results from [LLAHBF92] and [LLAHBF98].

(5.4) Flat Base Change. If $\varphi$ is flat, then the functors $-\otimes_{R}^{\mathbf{L}} S$ and $-\otimes_{R} S$ are naturally isomorphic, and we shall not distinguish between them. If $\varphi$ is also local, then it makes $S$ a faithfully flat $R$-module, so the amplitude of an $R$-complex does not change under base change. In this case, behavior of the Cohen-Macaulay defect is governed by the closed fiber $S / \mathfrak{m} S$ : for $Z \in \mathcal{D}_{\mathrm{b}}^{\mathrm{f}}(R)$ there is an equality [LLAHBF98, (1.2)]:

$$
\operatorname{cmd}_{S}\left(Z \otimes_{R} S\right)=\operatorname{cmd}_{R} Z+\operatorname{cmd} S / \mathfrak{m} S .
$$

(5.5) Bass Series. For a local homomorphism $\varphi$ of finite flat dimension a Bass series $\mathrm{I}_{\varphi}(t)$ can be defined (see [LLAHBF92, (2.1)] but also [LLAHBF97, (7.2)]). It is a formal Laurant series with non-negative integer coefficients, and for $Z \in \mathcal{D}_{\mathrm{b}}^{\mathrm{f}}(R)$ there is an equality of formal Laurant series [LLAHBF92, (2.2)]:

$$
\mathrm{I}_{S}^{Z \otimes_{R}^{\mathrm{L}} S}(t)=\mathrm{I}_{R}^{Z}(t) \mathrm{I}_{\varphi}(t) .
$$

Applying the equality to $Z=R$ we see that $\operatorname{ord} \mathrm{I}_{\varphi}(t)=\operatorname{depth} S-\operatorname{depth} R$, cf. (1.7.3).

If $\varphi$ is flat, then $\mathrm{I}_{\varphi}(t)$ is the Bass series of the closed fiber $S / \mathfrak{m} S$, cf. [HBFAT77].

(5.6) Theorem. Let $C \in \mathcal{D}_{\mathrm{b}}^{\mathrm{f}}(R)$. If $\varphi$ is local and flat, then the next two conditions are equivalent.

(i) $C$ is semi-dualizing for $R$.

(ii) $C \otimes_{R} S$ is semi-dualizing for $S$.

Furthermore, the following hold:

(a) $\operatorname{amp}\left(C \otimes_{R} S\right)=\operatorname{amp} C$; and 
(b) $\mathrm{I}_{S}^{C \otimes_{R} S}(t)=\mathrm{I}_{R}^{C}(t) \mathrm{I}_{S / \mathfrak{m} S}(t)$.

Proof. Since $S$ is flat we have $C \otimes_{R} S \simeq C \otimes_{R}^{\mathbf{L}} S$, and this complex belongs to $\mathcal{D}_{\mathrm{b}}^{\mathrm{f}}(S)$ by (1.3.1). By (1.5.7) the evaluation morphism $\omega_{C C S}$ is invertible, and the equivalence of $(i)$ and $(i i)$ follows from the commutative diagram below, as $S$ is faithfully flat over $R$.

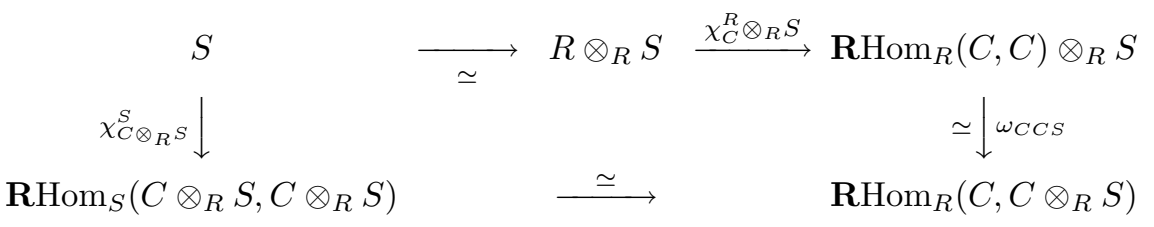

The bottom row isomorphism is (1.5.5).

Also (a) follows by faithful flatness, and (b) is (5.5.1).

(5.7) Proposition. Let $C$ be a semi-dualizing complex for $R$. If $\varphi$ is local and of finite flat dimension, then the base changed complex $C \otimes_{R}^{\mathrm{L}} S$ is semi-dualizing for $S$, and the following hold:

(a) $\operatorname{amp}\left(C \otimes_{R}^{\mathbf{L}} S\right)=\operatorname{amp} C$; and

(b) $\mathrm{I}_{S}^{C} \otimes_{R}^{\mathrm{L}} S(t)=\mathrm{I}_{R}^{C}(t) \mathrm{I}_{\varphi}(t)$.

Proof. It follows from (4.4) and (5.1) that $C \otimes_{R}^{\mathbf{L}} S$ is a semi-dualizing complex for $S$, and the equality in (b) is (5.5.1). By (5.1) we have $\operatorname{amp}\left(C \otimes_{R}^{\mathrm{L}} S\right) \leq \operatorname{amp} C$, and the opposite inequality is (5.13)(a); this proves (a).

The next three results explore the ascent and descent properties of Auslander categories under homomorphisms of finite flat dimension.

(5.8) Proposition. Let $C$ be a semi-dualizing complex for $R$. If $\varphi$ is of finite flat dimension, then $C \otimes_{R}^{\mathbf{L}} S$ is semi-dualizing for $S$, and for $X \in \mathcal{D}(R)$ we can consider the two statements:

$$
\text { (i) } X \in{ }_{C} \mathcal{A}(R) ; \quad \text { and } \quad(\text { ii }) \quad X \otimes_{R}^{\mathbf{L}} S \in_{C \otimes_{R}^{\mathbf{L}} S} \mathcal{A}(S)
$$

The following hold:

(a) (i) implies (ii); and

(b) if $\varphi$ is faithfully flat, then the two conditions are equivalent.

(5.9) Proposition. Let $C$ be a semi-dualizing complex for $R$. If $\varphi$ is of finite flat dimension, then $C \otimes_{R}^{\mathrm{L}} S$ is semi-dualizing for $S$, and for $Y \in \mathcal{D}(R)$ we can consider the two statements:

$$
\text { (i) } Y \in{ }_{C} \mathcal{B}(R) ; \quad \text { and } \quad(\text { ii }) Y \otimes_{R}^{\mathbf{L}} S \in_{C \otimes_{R}^{\mathrm{L}} S} \mathcal{B}(S) .
$$

The following hold:

(a) (i) implies (ii); and

(b) if $\varphi$ is faithfully flat, then the two conditions are equivalent. 
(5.10) Theorem. Let $C$ be a semi-dualizing complex for $R$. If $\varphi$ is of finite flat dimension, then $C \otimes_{R}^{\mathbf{L}} S$ is semi-dualizing for $S$, and for $Z \in \mathcal{D}_{\mathrm{b}}^{\mathrm{f}}(R)$ we can consider the two statements:

$$
\text { (i) } Z \in{ }_{C} \mathcal{R}(R) ; \quad \text { and } \quad(i i) Z \otimes_{R}^{\mathbf{L}} S \in_{C \otimes_{R}^{\mathrm{L}} S} \mathcal{R}(S)
$$

The following hold:

(a) (i) implies (ii);

(b) if $\varphi$ is faithfully flat, then (ii) and (i) are equivalent; and

(c) if $\varphi$ is local, then the next equality holds for $Z \in{ }_{C} \mathcal{R}(R)$ :

$$
G-\operatorname{dim}_{C \otimes_{R}^{\mathrm{L}} S}\left(Z \otimes_{R}^{\mathrm{L}} S\right)=G-\operatorname{dim}_{C} Z .
$$

The next result describes the behavior of $\mathrm{G}$-dimension under flat extensions, it generalizes [LLAHBF97, (4.1.4)].

(5.11) Corollary. Let $C$ be a semi-dualizing complex for $R$. If $\varphi$ is local and flat, then $C \otimes_{R} S$ is semi-dualizing for $S$, and for $Z \in \mathcal{D}_{\mathrm{b}}^{\mathrm{f}}(R)$ there is an equality:

$$
G-\operatorname{dim}_{C \otimes_{R} S}\left(Z \otimes_{R} S\right)=G-\operatorname{dim}_{C} Z .
$$

In particular, the two dimensions are simultaneously finite.

Proof. The complex $C \otimes_{R} S$ is semi-dualizing for $S$ by (5.6). It follows by the definition (3.11) and (5.10) (b) that $G-\operatorname{dim}_{C \otimes_{R} S}\left(Z \otimes_{R} S\right)$ is finite if and only if $G-\operatorname{dim}_{C} Z$ is so, and the equality follows by (5.10) (c).

Proof of (5.10). We assume that $S \in \mathcal{F}(R)$ and $Z \in \mathcal{D}_{\mathrm{b}}^{\mathrm{f}}(R)$. It follows by (5.7) that $C \otimes{ }_{R}^{\mathrm{L}} S$ is a semi-dualizing complex for $S$; and $Z \otimes_{R}^{\mathrm{L}} S$ belongs to $\mathcal{D}_{\mathrm{b}}^{\mathrm{f}}(S)$ by (1.3.1) and (1.4.1). The isomorphisms in the next chain follow by (1.5.5) and (1.5.7).

$$
\begin{aligned}
\left(Z \otimes_{R}^{\mathrm{L}} S\right)^{\dagger_{C} \otimes_{R}^{\mathrm{L}} S} & =\operatorname{RHom}_{S}\left(Z \otimes_{R}^{\mathrm{L}} S, C \otimes_{R}^{\mathbf{L}} S\right) \\
& \simeq \operatorname{RHom}_{R}\left(Z, C \otimes_{R}^{\mathbf{L}} S\right) \\
& \simeq \operatorname{RHom}_{R}(Z, C) \otimes_{R}^{\mathbf{L}} S \\
& =Z^{\dagger_{C}} \otimes_{R}^{\mathbf{L}} S .
\end{aligned}
$$

(a): Suppose $Z \in{ }_{C} \mathcal{R}(R)$, then $Z^{\dagger_{C}} \in \mathcal{D}_{\mathrm{b}}^{\mathrm{f}}(R)$ and by (1.4.1) we conclude from (*) that $\left(Z \otimes \otimes_{R}^{\mathbf{L}} S\right)^{\dagger_{C} \otimes_{R}^{\mathrm{L}} S} \in \mathcal{D}_{\mathrm{b}}^{\mathrm{f}}(S)$. In the commutative diagram

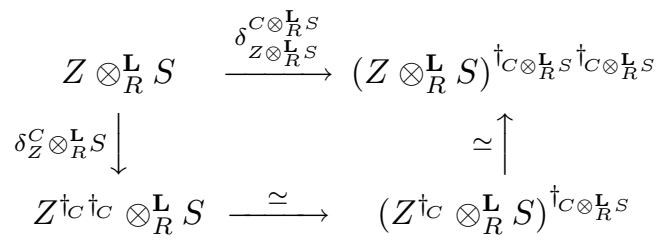

the unlabeled isomorphisms are (induced by) $(*)$; it shows that $\delta_{Z \otimes_{R}^{L} S}^{C \mathrm{~L}_{R} S}$ is invertible if $\delta_{Z}^{C}$ is so. We have now proved that $(i)$ implies (ii).

(b): Suppose $S$ is a faithfully flat $R$-module. Then it follows by $(*)$ that $Z^{\dagger_{C}}$ is bounded if and only if $\left(Z \otimes_{R}^{\mathbf{L}} S\right)^{\dagger_{C} \otimes_{R}^{\mathbf{L}} S}$ is so, and we see from the diagram that $\delta_{Z}^{C}$ is invertible if and only if $\delta_{Z \otimes_{R}^{\mathrm{L}} S}^{C \otimes^{\mathrm{L}} S}$ is so. 
(c): Suppose $\varphi$ is local and $Z \in{ }_{C} \mathcal{R}(R)$. It follows by property (a) that $G-\operatorname{dim}_{C \otimes \mathbb{R}_{R}}\left(Z \otimes_{R}^{\mathbf{L}} S\right)$ is finite, and the equality of G-dimensions follows by (3.14) and (5.5.1):

$$
\begin{aligned}
G-\operatorname{dim}_{C \otimes_{R}^{\mathbf{L}} S}\left(Z \otimes_{R}^{\mathbf{L}} S\right) & =\operatorname{depth} S-\operatorname{depth}_{S}\left(Z \otimes_{R}^{\mathbf{L}} S\right) \\
& =\operatorname{depth} S-\left(\operatorname{depth}_{R} Z+(\operatorname{depth} S-\operatorname{depth} R)\right) \\
& =\operatorname{depth} R-\operatorname{depth}_{R} Z \\
& =G-\operatorname{dim}_{C} Z . \quad \square
\end{aligned}
$$

The next proposition extends the results in (5.4) to homomorphisms of finite flat dimension; part (a) was used in the proof of (5.7)(a). Note that the inequality $\mathrm{cmd} R \leq \mathrm{cmd} S$, [LLAHBF98, (4.3)], for local homomorphisms of finite flat dimension, is a corollary to part (b). For the proof we need the following:

(5.12) Lemma. Let $\varphi$ be local and finite. If $Z \in \mathcal{D}_{\mathrm{b}}^{\mathrm{f}}(S)$, then $Z$ belongs to $\mathcal{D}_{\mathrm{b}}^{\mathrm{f}}(R)$, and there is an equality of Cohen-Macaulay defects:

$$
\operatorname{cmd}_{S} Z=\operatorname{cmd}_{R} Z \text {. }
$$

Proof. If $\varphi$ is local and finite, then so is the completion $\widehat{\varphi}: \widehat{R} \rightarrow \widehat{S}$. By (5.4.1) the Cohen-Macaulay defect is not affected by completion, so we may assume that $R$ is complete and hence possesses a dualizing complex $D$, cf. (1.8.3). Let $Z \in \mathcal{D}_{\mathrm{b}}^{\mathrm{f}}(S)$, it follows by finiteness of $\varphi$ that $Z \in \mathcal{D}_{\mathrm{b}}^{\mathrm{f}}(R)$. The complex $\mathbf{R H o m}_{R}(S, D)$ is dualizing for $S$, cf. [HHA, Chapter 15] or (6.2), and

$$
\begin{aligned}
\operatorname{cmd}_{S} Z & =\operatorname{amp}\left(\mathbf{R H o m}_{S}\left(Z, \mathbf{R H o m}_{R}(S, D)\right)\right) & & \text { by }(3.8) \\
& =\operatorname{amp}\left(\mathbf{R} \operatorname{Hom}_{R}(Z, D)\right) & & \text { by }(1.5 .6) \\
& =\operatorname{cmd}_{R} Z & & \text { by (3.8) }
\end{aligned}
$$

(5.13) Proposition. If $\varphi$ is local and of finite flat dimension, then the next two inequalities hold for $Z \in \mathcal{D}_{\mathrm{b}}^{\mathrm{f}}(R)$.

$$
\begin{aligned}
\operatorname{amp}\left(Z \otimes_{R}^{\mathrm{L}} S\right) & \geq \operatorname{amp} Z ; \quad \text { and } \\
\operatorname{cmd}_{S}\left(Z \otimes_{R}^{\mathrm{L}} S\right) & \geq \operatorname{cmd}_{R} Z .
\end{aligned}
$$

Proof. Let $Z \in \mathcal{D}_{\mathrm{b}}^{\mathrm{f}}(R)$. First we deal with the special case of a finite homomorphism, and then we deal with the general case.

If $\varphi$ is finite, then $S \in \mathcal{P}_{0}^{\mathrm{f}}(R)$, so (a) is Iversen's Amplitude Inequality (3.6.2). The inequality in (b) is a consequence of the New Intersection Theorem (3.6.1):

$$
\operatorname{cmd}_{S}\left(Z \otimes_{R}^{\mathbf{L}} S\right)=\operatorname{cmd}_{R}\left(Z \otimes_{R}^{\mathbf{L}} S\right) \geq \operatorname{cmd}_{R} Z ;
$$

the equality is (5.12), it applies as $Z \otimes_{R}^{\mathrm{L}} S \in \mathcal{D}_{\mathrm{b}}^{\mathrm{f}}(S)$ by (1.3.1) and (1.4.1).

We denote the maximal ideals of $R$ and $S$ by, respectively, $\mathfrak{m}$ and $\mathfrak{n}$. If $\varphi$ is not finite, we consider a Cohen factorization $R \stackrel{\dot{\varphi}}{\longrightarrow} R^{\prime} \stackrel{\varphi^{\prime}}{\longrightarrow} \widehat{S}$ of the semi-completion $\grave{\varphi}: R \rightarrow \widehat{S}$ (the composition of $\varphi$ and the the canonical map from $S$ to the $\mathfrak{n}$-adic completion $\widehat{S}$ ). That is, $R^{\prime}$ is complete, $R^{\prime} / \mathfrak{m} R^{\prime}$ is regular, and we have $\grave{\varphi}=\varphi^{\prime} \dot{\varphi}$, where $\dot{\varphi}$ is a flat local homomorphism and $\varphi^{\prime}$ is a finite local homomorphism with $\mathrm{fd} \varphi^{\prime}<\infty$; cf. [LLAHBFHr94, (1.1) and (3.3)]. In particular, there is an isomorphism of $\widehat{S}$-complexes:

$$
Z \otimes_{R}^{\mathbf{L}} \widehat{S} \simeq\left(Z \otimes_{R}^{\mathbf{L}} R^{\prime}\right) \otimes_{R^{\prime}}^{\mathbf{L}} \widehat{S}
$$


Now, we have

$$
\begin{aligned}
\operatorname{cmd}_{S}\left(Z \otimes_{R}^{\mathbf{L}} S\right) & =\operatorname{cmd}_{\widehat{S}}\left(\left(Z \otimes_{R}^{\mathbf{L}} S\right) \otimes_{S} \widehat{S}\right) & & \text { by (5.4.1) } \\
& =\operatorname{cmd}_{\widehat{S}}\left(Z \otimes_{R}^{\mathbf{L}} \widehat{S}\right) & & \text { by (1.5.4) } \\
& =\operatorname{cmd}_{\widehat{S}}\left(\left(Z \otimes_{R}^{\mathbf{L}} R^{\prime}\right) \otimes_{R^{\prime}}^{\mathbf{L}} \widehat{S}\right) & & \text { by }(*) \\
& \geq \operatorname{cmd}_{R^{\prime}}\left(Z \otimes_{R}^{\mathbf{L}} R^{\prime}\right) & & \text { as } \varphi^{\prime} \text { is finite } \\
& =\operatorname{cmd}_{R} Z & & \text { by (5.4.1). }
\end{aligned}
$$

This proves (b), and the proof of (a) is similar, only easier.

Proof of (5.1). (i) $\Rightarrow$ (ii): Suppose $S \in{ }_{C} \mathcal{A}(R)$, then $C \otimes_{R}^{\mathbf{L}} S \in \mathcal{D}_{\mathrm{b}}^{\mathrm{f}}(S)$, cf. (1.3.1), and the commutative diagram below shows that the homothety morphism $\chi_{C \otimes_{R}^{S} S}^{S}$ is invertible.

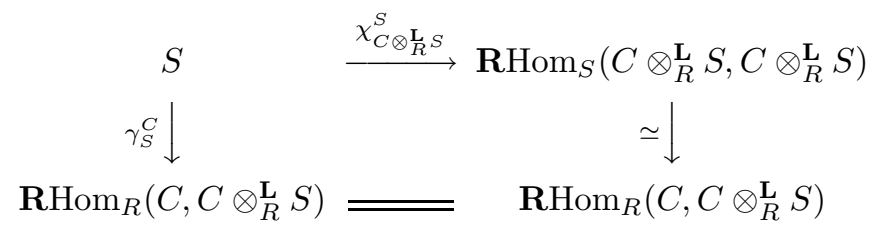

The unlabeled isomorphism is (1.5.5).

$(i i) \Rightarrow(i)$ : If $C \otimes_{R}^{\mathbf{L}} S$ is semi-dualizing for $S$, then, in particular, $C \otimes_{R}^{\mathbf{L}} S \in \mathcal{D}_{\mathrm{b}}(R)$ and the diagram above shows that $\gamma_{S}^{C}$ is an isomorphism.

The inequality of amplitudes, (a), follows from (4.8) (b) and the equality of formal Laurant series, (b), from (3.18.2) and (1.7.6):

$$
\mathrm{I}_{S}(t)=\mathrm{P}_{C \otimes \otimes_{R} S}^{S}(t) \mathrm{I}_{S}^{C \otimes_{R}^{\mathrm{L}} S}(t)=\mathrm{P}_{C}^{R}(t) \mathrm{I}_{S}^{C \otimes_{R}^{\mathrm{L}} S}(t) .
$$

In the following $E$ is a dualizing complex for $S$. We have

$$
\left(C \otimes_{R}^{\mathbf{L}} S\right)^{\dagger_{E}}=\mathbf{R H o m}_{S}\left(C \otimes_{R}^{\mathbf{L}} S, E\right) \simeq \mathbf{R H o m}_{R}(C, E),
$$

by (1.5.5), and this isomorphism shows that the equivalence of $(i i)$ and $(i v)$ is simply (2.12). It follows by (5.3) (d) (which only uses the implication $(i) \Rightarrow(i i)$ of (5.1)) that (i) implies (iii); this leaves us only one implication to prove.

(iii) $\Rightarrow($ iv $)$ : We assume that $E$ belongs to ${ }_{C} \mathcal{B}(R)$; the complex $\mathbf{R H o m}_{R}(C, E)$ is then bounded, i.e., it belongs to $\mathcal{D}_{\mathrm{b}}^{\mathrm{f}}(S)$ as desired, cf. (1.3.2). The unlabeled isomorphism in the commutative diagram

$$
\begin{aligned}
& S \stackrel{\chi_{\mathbf{R H o m}}^{S}(C, E)}{\longrightarrow} \mathbf{R H o m}_{S}\left(\mathbf{R H o m}_{R}(C, E), \mathbf{R H o m}_{R}(C, E)\right) \\
& \chi_{E}^{S} \downarrow \simeq \quad \simeq \downarrow \\
& \mathbf{R H o m}_{S}(E, E) \underset{\mathbf{R} \operatorname{Hom}_{S}\left(\xi_{E}^{C}, E\right)}{\simeq} \quad \mathbf{R H o m}_{S}\left(C \otimes_{R}^{\mathbf{L}} \mathbf{R} \operatorname{Hom}_{R}(C, E), E\right)
\end{aligned}
$$

follows by 1.5.5), (1.5.2), and (1.5.4):

$$
\begin{aligned}
\mathbf{R H o m}_{S}\left(\mathbf{R H o m}_{R}(C, E)\right. & \left., \mathbf{R} \operatorname{Hom}_{R}(C, E)\right) \\
& \simeq \mathbf{R} \operatorname{Hom}_{S}\left(\mathbf{R} H_{R}(C, E), \mathbf{R} \operatorname{Hom}_{S}\left(C \otimes_{R}^{\mathbf{L}} S, E\right)\right) \\
& \simeq \mathbf{R H o m}_{S}\left(\mathbf{R} H_{R}(C, E) \otimes_{S}^{\mathbf{L}}\left(C \otimes_{R}^{\mathbf{L}} S\right), E\right) \\
& \simeq \mathbf{R H o m}_{S}\left(C \otimes_{R}^{\mathbf{L}} \mathbf{R} \operatorname{Hom}_{R}(C, E), E\right) .
\end{aligned}
$$


It follows that the homothety morphism $\chi_{\mathbf{R} \operatorname{Hom}_{R}(C, E)}^{S}$ is invertible, and the proof is complete.

Proof of (5.3). It follows by (5.1) that $C \otimes_{R}^{\mathbf{L}} S$ is semi-dualizing for $S$. By (1.5.4) and $(1.5 .5)$ we have

$$
\begin{aligned}
\left(C \otimes_{R}^{\mathbf{L}} S\right) \otimes_{S}^{\mathbf{L}} X \in \mathcal{D}_{\mathrm{b}}(S) & \Longleftrightarrow C \otimes_{R}^{\mathbf{L}} X \in \mathcal{D}_{\mathrm{b}}(R) ; \quad \text { and } \\
\mathbf{R H o m}_{S}\left(C \otimes_{R}^{\mathbf{L}} S, Y\right) \in \mathcal{D}_{\mathrm{b}}(S) & \Longleftrightarrow \mathbf{R} H_{R}(C, Y) \in \mathcal{D}_{\mathrm{b}}(R) .
\end{aligned}
$$

These standard isomorphisms also account for the unlabeled isomorphisms in the diagrams below, and since they only involve natural maps, commutativity is easily checked.

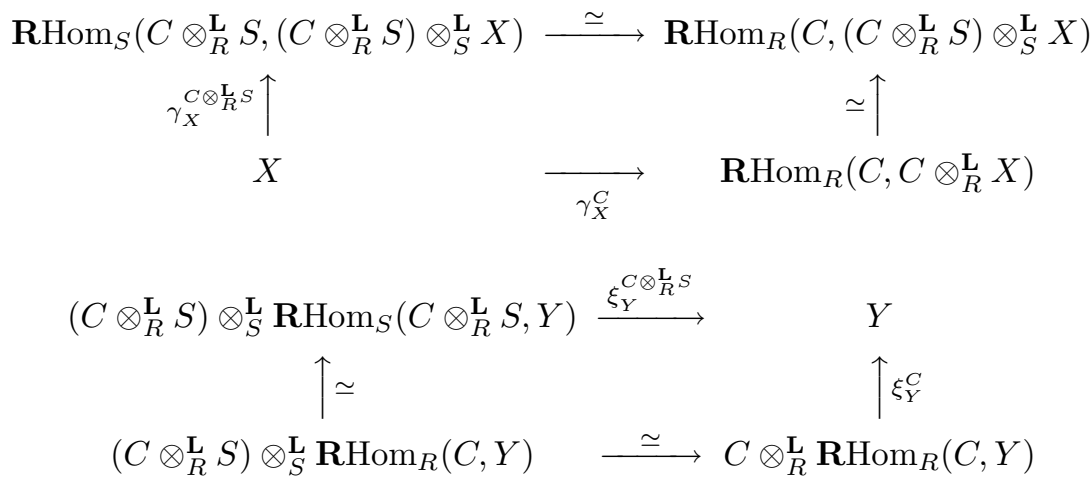

This establishes (a) and (b), and the full embeddings follow in view of (4.4).

Proof of (5.8) and (5.9). We assume that $S \in \mathcal{F}(R)$; then $C \otimes_{R}^{\mathrm{L}} S$ is a semidualizing complex for $S$ by (5.7). Note that for $X \in \mathcal{D}(R)$ we have

$$
\left(C \otimes_{R}^{\mathbf{L}} S\right) \otimes_{S}^{\mathbf{L}}\left(X \otimes_{R}^{\mathbf{L}} S\right) \simeq C \otimes_{R}^{\mathbf{L}}\left(X \otimes_{R}^{\mathbf{L}} S\right) \simeq\left(C \otimes_{R}^{\mathbf{L}} X\right) \otimes_{R}^{\mathbf{L}} S .
$$

Also note that, when $C \otimes_{R}^{\mathbf{L}} X$ is bounded, we have the following commutative diagram:

$$
\begin{aligned}
& \mathbf{R H o m}_{S}\left(C \otimes_{R}^{\mathbf{L}} S,\left(C \otimes_{R}^{\mathbf{L}} S\right) \otimes_{S}^{\mathbf{L}}\left(X \otimes_{R}^{\mathbf{L}} S\right)\right) \stackrel{\simeq}{\longrightarrow} \operatorname{RHom}_{R}\left(C,\left(C \otimes_{R}^{\mathbf{L}} X\right) \otimes_{R}^{\mathbf{L}} S\right) \\
& \gamma_{X \otimes R_{R}^{L} S}^{C \otimes}{ }^{\mathrm{L}} S \uparrow \\
& X \otimes \otimes_{R}^{\mathbf{L}} S \\
& \begin{array}{c}
\simeq \uparrow_{C\left(C \otimes_{R} \mathbf{L} X\right) S} \\
\underset{\gamma_{X}^{C} \otimes_{R}^{\mathbf{L} S}}{\longrightarrow} \operatorname{RHom}_{R}\left(C, C \otimes_{R}^{\mathbf{L}} X\right) \otimes_{R}^{\mathbf{L}} S
\end{array}
\end{aligned}
$$

The unlabeled isomorphism is induced by (1.5.4) and (*), and the evaluation morphism is invertible by (1.5.7).

(a): If $X \in{ }_{C} \mathcal{A}(R)$, then $X$ is bounded and hence so is $X \otimes_{R}^{\mathbf{L}} S$, cf. (1.4.1). Also $C \otimes_{R}^{\mathbf{L}} X$ is bounded, and from $(*)$ we conclude that $\left(C \otimes_{R}^{\mathbf{L}} S\right) \otimes_{S}^{\mathbf{L}}\left(X \otimes_{R}^{\mathbf{L}} S\right) \in \mathcal{D}_{\mathrm{b}}(S)$. The diagram shows that $\gamma_{X \otimes_{R}^{\mathrm{L}} S}^{C \otimes^{\mathrm{L}} S}$ is invertible since $\gamma_{X}^{C}$ is so, and hence $X \otimes_{R}^{\mathrm{L}} S \in$ $C \otimes{ }_{R}{ }_{S} \mathcal{A}(S)$ as desired.

(b): If $X \otimes_{R}^{\mathbf{L}} S \in{ }_{C \otimes{ }_{R} S} \mathcal{A}(S)$ we conclude by faithful flatness of $S$ over $R$ that both $X$ and $C \otimes_{R}^{\mathrm{L}} X$ (in view of $(*)$ ) must belong to $\mathcal{D}_{\mathrm{b}}(R)$. The diagram now shows that the induced map $\gamma_{X}^{C} \otimes_{R}^{\mathrm{L}} S$, and hence also $\gamma_{X}^{C}$, is invertible. This concludes the proof of (5.8), and the proof of (5.9) is analogous. 


\section{Finite Local Homomorphisms}

In this section $\varphi: R \rightarrow S$ is a finite local homomorphism of rings.

We study induced semi-dualizing complexes for $S$ of the form $\operatorname{RHom}_{R}(S, C)$, where $C$ is semi-dualizing for $R$. The motivation for doing so, of course, comes from RAD.

The proofs of (6.1) and (6.4) are deferred to the end of the section.

(6.1) Theorem. Let $C$ be a semi-dualizing complex for $R$. The next two conditions are equivalent.

(i) $S \in{ }_{C} \mathcal{R}(R)$.

(ii) $\mathbf{R H o m}_{R}(S, C)$ is semi-dualizing for $S$.

And there is an equality of Bass series:

$$
\mathrm{I}_{S}^{\mathbf{R H o m}}(S, C)(t)=\mathrm{I}_{R}^{C}(t) .
$$

When the equivalent conditions $(i)$ and (ii) are satisfied, there is also an inequality:

$$
\operatorname{cmd}_{S} \mathbf{R H o m}_{R}(S, C) \leq \operatorname{cmd}_{R} C .
$$

We start by spelling out some easy consequences of the theorem. The corollary below is a well-known result, cf. [HHA, Chapter 15], and it follows immediately from the theorem by (1.8.1).

(6.2) Corollary. If $D$ is a (normalized) dualizing complex for $R$, then $\mathbf{R H o m}_{R}(S, D)$ is a (normalized) dualizing complex for $S$.

(6.3) Observation. If the complex $\operatorname{RHom}_{R}(S, R)$ is semi-dualizing for $S$, then $S \in{ }_{R} \mathcal{R}(R)$ by (6.1), that is, $\varphi$ makes $S$ an $R$-module of finite Gorenstein dimension (in the sense of [MAu67]). If $\operatorname{RHom}_{R}(S, R)$ is dualizing for $S$, then $1=\mathrm{I}_{S}^{\mathbf{R H o m}}(S, R)(t)=\mathrm{I}_{R}(t)$ by (1.8.1) and (6.1) (b), so $R$ is Gorenstein, cf. (1.8.2), and by MAuMBr69, (4.20)] we have $G-\operatorname{dim}_{R} M<\infty$ for all finite $R$-modules $M$ (see also (8.5) ).

(6.4) Proposition. Let $C$ be a semi-dualizing complex for $R$. If $\varphi$ is of finite flat dimension, then the complex $\operatorname{RHom}_{R}(S, C)$ is semi-dualizing for $S$, and the following hold:

(a) $\operatorname{cmd}_{S} \mathbf{R} \operatorname{Hom}_{R}(S, C)=\operatorname{cmd}_{R} C$; and

(b) $\operatorname{amp}(\mathbf{R H o m} R(S, C)) \geq \operatorname{amp} C$.

The next result generalizes and extends [EGl84, Proposition 5]; applying it to $C=R$ we recover [LLAHBF97, (7.11)].

(6.5) Theorem. Let $C$ be a semi-dualizing complex for $R$, and assume $G$ - $\operatorname{dim}_{C} S$ is finite. Then the complex $S^{\dagger_{C}}=\operatorname{RHom}_{R}(S, C)$ is semi-dualizing for $S$, and for $Z \in \mathcal{D}_{\mathrm{b}}^{\mathrm{f}}(S)$ there is an equality:

$$
G-\operatorname{dim}_{C} Z=G-\operatorname{dim}_{C} S+G-\operatorname{dim}_{S^{\dagger}} Z .
$$

In particular, $G-\operatorname{dim}_{S^{\dagger} C} Z$ and $G-\operatorname{dim}_{C} Z$ are simultaneously finite, that is,

$$
Z \in{ }_{S^{\dagger_{C}}} \mathcal{R}(S) \quad \Longleftrightarrow \quad Z \in{ }_{C} \mathcal{R}(R)
$$


Proof. By definition, (3.11), finiteness of $G-\operatorname{dim}_{C} S$ is tantamount to $S$ being $C-$ reflexive. It, therefore, follows by (6.1) that $S^{\dagger_{C}}=\mathbf{R} \operatorname{Hom}_{R}(S, C)$ is a semi-dualizing complex for $S$. We first prove that the two G-dimensions are simultaneously finite. Let $Z \in \mathcal{D}_{\mathrm{b}}^{\mathrm{f}}(S)$; since $\varphi$ is finite $Z$ belongs to $\mathcal{D}_{\mathrm{b}}^{\mathrm{f}}(R)$, and by (1.5.6) we have

$$
\begin{aligned}
Z^{\left.\dagger_{\left(S^{\dagger} C\right.}\right)} & =\mathbf{R H o m}_{S}\left(Z, S^{\dagger_{C}}\right) \\
& =\mathbf{R} \operatorname{Hom}_{S}\left(Z, \mathbf{R} \operatorname{Hom}_{R}(S, C)\right) \\
& \simeq \mathbf{R H o m}_{R}(Z, C) \\
& =Z^{\dagger_{C}}
\end{aligned}
$$

so $Z^{\dagger}{ }_{\left(S^{\dagger} C\right)}$ belongs to $\mathcal{D}_{\mathrm{b}}^{\mathrm{f}}(S)$ if and only if $Z^{\dagger_{C}} \in \mathcal{D}_{\mathrm{b}}^{\mathrm{f}}(R)$. It now follows by the commutative diagram below that the complex $Z$ belongs to ${ }_{S^{\dagger}} \mathcal{R}(S)$ if and only if it belongs to ${ }_{C} \mathcal{R}(R)$.

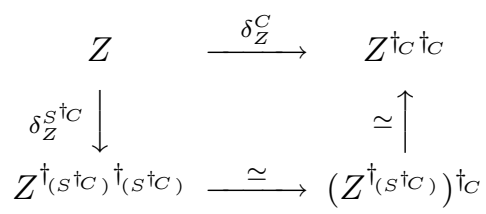

The unlabeled isomorphisms are induced by $(*)$.

Finally, for $Z \in_{S^{\dagger} C} \mathcal{R}(S)$ we have

$$
\begin{aligned}
G-\operatorname{dim}_{S^{\dagger C}} Z & =\inf S^{\dagger_{C}}-\inf Z^{\left.\dagger_{\left(S^{\dagger} C\right.}\right)} \\
& =\inf C-\inf Z^{\dagger_{C}}-\left(\inf C-\inf S^{\dagger_{C}}\right) \text { by }(*) \\
& =G-\operatorname{dim}_{C} Z-G-\operatorname{dim}_{C} S . \quad \square
\end{aligned}
$$

There is also a descent result for complexes with non-finite homology modules:

(6.6) Proposition. Let $C$ be a semi-dualizing complex for $R$. If $\varphi$ is of finite flat dimension, then $S^{\dagger_{C}}=\mathbf{R H o m}_{R}(S, C)$ is a semi-dualizing complex for $S$, and the next implication holds for $X \in \mathcal{D}(R)$ :

$$
X \in{ }_{C} \mathcal{A}(R) \quad \Longrightarrow \quad X \otimes_{R}^{\mathbf{L}} S \in{ }_{S^{\dagger C}} \mathcal{A}(S)
$$

Proof. We assume that $\varphi$ has finite flat dimension, i.e., $S \in \mathcal{P}_{0}^{\mathrm{f}}(R)$, then it follows by (6.4) that $S^{\dagger_{C}}=\mathbf{R} \operatorname{Hom}_{R}(S, C)$ is semi-dualizing for $S$. Since $X$ is bounded so is $X \otimes_{R}^{\mathbf{L}} S$, cf. (1.4.1). Also $C \otimes_{R}^{\mathrm{L}} X$ is bounded, so it follows by (1.4.3) and the next chain of isomorphisms that $S^{\dagger_{C}} \otimes_{S}^{\mathbf{L}}\left(X \otimes_{R}^{\mathbf{L}} S\right) \in \mathcal{D}_{\mathrm{b}}(S)$.

$$
\begin{aligned}
& S^{\dagger_{C}} \otimes_{S}^{\mathbf{L}}\left(X \otimes_{R}^{\mathbf{L}} S\right) \simeq S^{\dagger_{C}} \otimes_{S}^{\mathbf{L}}\left(S \otimes_{R}^{\mathbf{L}} X\right) \\
& \simeq \mathbf{R H o m}_{R}(S, C) \otimes_{R}^{\mathbf{L}} X \\
& \simeq \mathbf{R H o m}_{R}\left(S, C \otimes_{R}^{\mathbf{L}} X\right) \text { by (1.5.7). }
\end{aligned}
$$


Finally, the commutative diagram

$$
\begin{aligned}
& \mathbf{R H o m}_{S}\left(S^{\dagger_{C}},\left(S^{\dagger_{C}} \otimes_{S}^{\mathbf{L}}\left(X \otimes_{R}^{\mathbf{L}} S\right)\right)\right) \stackrel{\simeq}{\longrightarrow} \operatorname{RHom}_{S}\left(S^{\dagger_{C}}, \mathbf{R H o m}_{R}\left(S, C \otimes_{R}^{\mathbf{L}} X\right)\right) \\
& \gamma_{X \otimes \mathbb{R}^{\mathrm{L}} S}^{\mathrm{S}^{\dagger} \mathrm{C}} \uparrow \\
& X \otimes \otimes_{R}^{\mathbf{L}} S \\
& \gamma_{X}^{C} \otimes_{R}^{\mathrm{L}} S \downarrow \simeq \\
& \simeq \\
& \mathbf{R H o m}_{R}\left(\mathbf{R H o m}{ }_{R}(S, C), C \otimes_{R}^{\mathbf{L}} X\right) \\
& \mathbf{R H o m}_{R}\left(C, C \otimes \otimes_{R}^{\mathbf{L}} X\right) \otimes_{R}^{\mathbf{L}} S \\
& \simeq \theta_{S C\left(C \otimes \mathbb{L}_{R} X\right)}
\end{aligned}
$$

shows that $\gamma_{X \otimes{ }_{R} S}^{S^{\dagger C}}$ is an isomorphism. The top row isomorphism is induced by $(*)$, the unlabeled vertical isomorphism follows by (1.5.6), and the evaluation morphism $\theta_{S C\left(C \otimes \otimes_{R}^{\mathrm{L} X)}\right.}$ is invertible by (1.5.8).

(6.7) Observation. Let $C$ be a semi-dualizing complex for $R$, and assume that $R$ has a dualizing complex $D$. If $S$ belongs to ${ }_{C} \mathcal{R}(R)$ it also belongs to ${ }_{C^{\dagger}} \mathcal{A}(R)$, cf. (4.7), so by (6.1) and (5.1) the complexes $\mathbf{R H o m}_{R}(S, C)$ and $C^{\dagger_{D}} \otimes_{R}^{\mathrm{L}} S$ are both semi-dualizing for $S$. By the next chain of isomorphisms, they are the dagger duals of each other with respect to the dualizing complex $E=\mathbf{R H o m}_{R}(S, D)$ for $S$, cf. (6.2).

$$
\begin{array}{rlrl}
\mathbf{R H o m}_{R}(S, C)^{\dagger_{E}} & =\mathbf{R H o m}_{S}\left(\mathbf{R H o m}_{R}(S, C), \mathbf{R} \operatorname{Hom}_{R}(S, D)\right) & \\
& \simeq \mathbf{R} \operatorname{Hom}_{R}\left(\mathbf{R} H_{R}(S, C), D\right) & & \text { by (1.5.6) } \\
& \simeq S \otimes_{R}^{\mathbf{L}} \mathbf{R} \operatorname{Hom}_{R}(C, D) & & \text { by (1.5.8) } \\
& \simeq C^{\dagger_{D}} \otimes_{R}^{\mathbf{L}} S . & &
\end{array}
$$

If $S$ belongs to ${ }_{C} \mathcal{R}(R)$ and to ${ }_{C} \mathcal{A}(R)$ (e.g., $S \in \mathcal{P}_{0}^{\mathrm{f}}(R)$ ), then, by (2.12), the pair $\left(C, C^{\dagger_{D}}\right)$ of semi-dualizing complexes for $R$ gives rise to two pairs of semi-dualizing complexes for $S$, namely

$\mathbf{R H o m}_{R}(S, C)$ and its dagger dual $\mathbf{R} \operatorname{Hom}_{R}(S, C)^{\dagger_{E}} \simeq C^{\dagger_{D}} \otimes_{R}^{\mathbf{L}} S ;$ and

$\mathbf{R H o m}_{R}\left(S, C^{\dagger_{D}}\right)$ and its dagger dual $\mathbf{R} \operatorname{Hom}_{R}\left(S, C^{\dagger_{D}}\right)^{\dagger_{E}} \simeq C \otimes_{R}^{\mathbf{L}} S$.

Proof of (6.1). The equality of Bass series, (a), follows by (1.7.9).

$(i) \Rightarrow(i i)$ : If $S \in{ }_{C} \mathcal{R}(R)$, then the dagger dual $S^{\dagger_{C}}=\mathbf{R H o m}_{R}(S, C)$ is bounded, so $\operatorname{RHom}_{R}(S, C) \in \mathcal{D}_{\mathrm{b}}^{\mathrm{f}}(S)$ as required, cf. (1.7.8). The unlabeled isomorphism in the commutative diagram

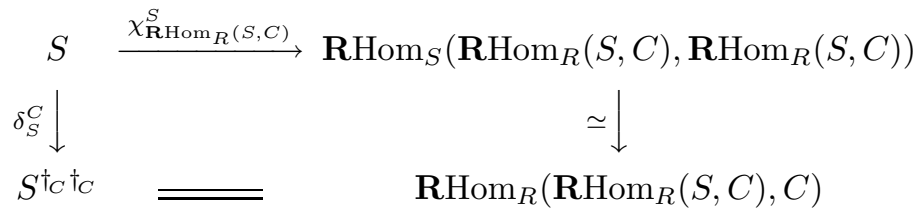

is (1.5.6); the diagram shows that the homothety morphism $\chi_{\mathbf{R} H_{0}}^{S}(S, C)$ is invertible since $\delta_{S}^{C}$ is so.

$(i i) \Rightarrow(i)$ : If the complex $\mathbf{R H o m}_{R}(S, C)=S^{\dagger_{C}}$ is semi-dualizing for $S$, then it belongs to $\mathcal{D}_{\mathrm{b}}^{\mathrm{f}}(S)$ and hence to $\mathcal{D}_{\mathrm{b}}^{\mathrm{f}}(R)$, as $\varphi$ is finite. The diagram above now shows that the biduality map $\delta_{S}^{C}$ is invertible, so $S \in{ }_{C} \mathcal{R}(R)$. 
When $S \in{ }_{C} \mathcal{R}(R)$ also the complex $S^{\dagger_{C}}=\operatorname{RHom}_{R}(S, C)$ belongs to ${ }_{C} \mathcal{R}(R)$, cf. (2.11), and the inequality of Cohen-Macaulay defects, (b), follows by applying (3.3) (a) to $S^{\dagger_{C}}$ and using (5.12).

Proof of (6.4). If $\mathrm{fd} \varphi<\infty$, then $S$ belongs to $\mathcal{P}_{0}^{\mathrm{f}}(R)$, so it follows by (2.9) and (6.1) that $\mathbf{R} \operatorname{Hom}_{R}(S, C)$ is semi-dualizing for $S$. By (6.1) (b) we have $\operatorname{cmd}_{S} \mathbf{R} \operatorname{Hom}_{R}(S, C)$ $\leq \operatorname{cmd}_{R} C$, and by (5.12) we have $\operatorname{cmd}_{S} \mathbf{R} \operatorname{Hom}_{R}(S, C)=\operatorname{cmd}_{R} \mathbf{R} \operatorname{Hom}_{R}(S, C)$, so (a) is proved by establishing the inequality $\operatorname{cmd}_{R} \mathbf{R H o m}_{R}(S, C) \geq \operatorname{cmd}_{R} C$. By (5.4.1) we are free to assume that $R$ is complete and, thereby, has a dualizing complex $D$, cf. (1.8.3). The desired inequality follows by the next calculation:

$$
\begin{array}{rlrl}
\operatorname{cmd}_{R} \mathbf{R H o m}_{R}(S, C) & =\operatorname{amp}(\mathbf{R H o m} & \\
& =\operatorname{amp}\left(\mathbf{R} \operatorname{Hom}_{R}\left(\mathbf{R} \operatorname{Hom}_{R}(S, C), D\right)\right) & & \\
& =\operatorname{amp}\left(S \otimes_{R}^{\mathbf{L}} \mathbf{R} \operatorname{Hom}_{R}(C, D)\right) & & \text { by (1.5.8) } \\
& =\operatorname{amp}\left(C^{\dagger_{D}} \otimes_{R}^{\mathbf{L}} S\right) & & \\
& \geq \operatorname{amp} C^{\dagger_{D}} & & \text { by (3.6.2) } \\
& =\operatorname{cmd}_{R} C & & \text { by (3.8). }
\end{array}
$$

The inequality in (b) follows by a similar calculation, only it uses (3.6.1) instead of (3.6.2).

\section{EXAMPLES}

In this section $\varphi: R \rightarrow S$ is a local homomorphism of rings.

We shall apply the results from the previous sections to give some examples of semi-dualizing complexes. First, we review the special semi-dualizing complexes used by L.L. Avramov and H.-B. Foxby in LLAHBF97, and we apply the main results from sections 5 and 6 in their setting to obtain converses to some key results in that paper. Next, in search of an appropriate invariant for telling semi-dualizing complexes apart, we make a short study of Gorenstein homomorphisms, and the so-called type emerges a suitable choice. The section closes with an answer to Golod's question: a recipe for constructing rings with any desired (finite) number of semi-dualizing complexes, or modules.

(7.1) Relative Dualizing Complexes. The concept of relative dualizing complexes, that is, dualizing complexes for local homomorphisms, was introduced and studied in [LLAHBF97]; we catalogue a few facts about these complexes:

A complex $A \in \mathcal{D}(S)$ is said to be dualizing for $\varphi$ if and only if

- $A$ is semi-dualizing for $S$; and

- $D^{\prime} \otimes_{\widehat{R}}^{\mathbf{L}}\left(A \otimes_{S} \widehat{S}\right) \in \mathcal{I}(\widehat{S})$ when $D^{\prime}$ is a dualizing complex for $\widehat{R}$.

If $R$ has a dualizing complex $D$, then this is equivalent to requiring that $A$ be semi-dualizing for $S$ and $D \otimes_{R}^{\mathrm{L}} A \in \mathcal{I}(S)$ [LLAHBF97, (5.2)(b)].

We say that $\varphi$ is of finite Gorenstein dimension [LLAHBF97, (4.3)] if $\widehat{S} \in$ $D^{\prime} \mathcal{A}(\widehat{R})$, where $D^{\prime}$ is a dualizing complex for $\widehat{R}$; this is certainly the case if $\mathrm{fd} \varphi<\infty$ [LLAHBF97, (4.4.2)]. For homomorphisms of finite Gorenstein dimension relative dualizing complexes often exist, and when they do, they are uniquely determined up to isomorphism and shift [LLAHBF97, (5.4)]. Examples: 
- If $\varphi$ is finite of finite Gorenstein dimension, i.e., $S \in{ }_{R} \mathcal{R}(R)$ cf. (4.7) and (5.11), then $\mathbf{R H o m}_{R}(S, R)$ is dualizing for $\varphi$ [LLAHBF97, (6.5)]. Conversely, by (6.1) $\varphi$ is of finite Gorenstein dimension if $\mathbf{R H o m}_{R}(S, R)$ is semi-dualizing for $S$, cf. (66.3)

- If $D$ and $E$ are dualizing complexes for $R$ and $S$, respectively, and $S \in$ ${ }_{D} \mathcal{A}(R)$, then $\mathbf{R H o m}_{R}(D, E)$ is a dualizing complex for $\varphi$ [LLAHBF97, (6.1)]. Conversely, by (5.1) $S$ belongs to $D \mathcal{A}(R)$ if $\mathbf{R H o m}_{R}(D, E)$ is semi-dualizing for $S$.

Let $\varphi$ be of finite flat dimension; recall that $\varphi$ is said to be Gorenstein (see [LLAHBF92]) if the Bass series $\mathrm{I}_{\varphi}(t)$ is a monomial. Suppose $R$ and $S$ have dualizing complexes $D$ and $E$, it was proved in [LLAHBF97 that $\varphi$ is Gorenstein if and only if $E \sim D \otimes_{R}^{\mathbf{L}} S$. This condition can be rewritten, clumsily, as $\left(R \otimes_{R}^{\mathbf{L}} S\right)^{\dagger_{E}} \sim R^{\dagger_{D}} \otimes_{R}^{\mathbf{L}} S$ to suggest that base change commutes with dagger duality; the next result makes this idea explicit.

(7.2) Theorem. Let $\varphi$ be local and of finite flat dimension. If $R$ has a dualizing complex $D$, then the next two conditions are equivalent.

(i) $\varphi$ is Gorenstein.

(ii) $D \otimes_{R}^{\mathbf{L}} S$ is dualizing for $S$.

And when $S$ has a dualizing complex $E$, they are equivalent with the following:

(iii) $\left(Z \otimes_{R}^{\mathbf{L}} S\right)^{\dagger_{E}} \sim Z^{\dagger_{D}} \otimes_{R}^{\mathbf{L}} S$ for all $Z \in \mathcal{D}_{\mathrm{b}}^{\mathrm{f}}(R)$.

(iv) $\left(C \otimes_{R}^{\mathbf{L}} S\right)^{\dagger_{E}} \sim C^{\dagger_{D}} \otimes_{R}^{\mathbf{L}} S$ for some semi-dualizing complex $C$ for $R$.

Proof. The equivalence of $(i)$ and $(i i)$ is immediate by (5.5.1) and (1.8.1), and known from [LLAHBF97, (7.8) and (7.7.1)]. In the following $E$ is a dualizing complex for $S$.

(ii) $\Rightarrow\left(\right.$ iii): Suppose $E \sim D \otimes_{R}^{\mathrm{L}} S$. For $Z \in \mathcal{D}_{\mathrm{b}}^{\mathrm{f}}(R)$ we have

$$
\begin{aligned}
\left(Z \otimes_{R}^{\mathbf{L}} S\right)^{\dagger_{E}} & =\mathbf{R H o m}_{S}\left(Z \otimes_{R}^{\mathbf{L}} S, E\right) \\
& \simeq \operatorname{RHom}_{R}(Z, E) \quad \text { by (1.5.5) } \\
& \sim \mathbf{R H o m}_{R}\left(Z, D \otimes_{R}^{\mathbf{L}} S\right) \\
& \simeq \operatorname{RHom}_{R}(Z, D) \otimes_{R}^{\mathbf{L}} S \quad \text { by (1.5.7) } \\
& =Z^{\dagger_{D}} \otimes_{R}^{\mathbf{L}} S .
\end{aligned}
$$

Condition (iii) is, obviously, stronger than $(i v)$, so we need only prove $(i v) \Rightarrow(i i)$ : Let $C$ be a semi-dualizing complex for $R$, and assume that $\left(C \otimes_{R}^{\mathbf{L}} S\right)^{\dagger_{E}} \sim C^{\dagger_{D}} \otimes_{R}^{\mathbf{L}} S$. Since, by (5.3) (d) and (4.4), both $E$ and $D$ belong to ${ }_{C} \mathcal{B}(R)$, we have

$$
\begin{aligned}
E & \simeq C \otimes_{R}^{\mathbf{L}} \mathbf{R H o m}_{R}(C, E) \\
& \simeq C \otimes_{R}^{\mathbf{L}} \mathbf{R} \operatorname{Hom}_{S}\left(C \otimes_{R}^{\mathbf{L}} S, E\right) \quad \text { by 11.5.5 } \\
& =C \otimes_{R}^{\mathbf{L}}\left(C \otimes_{R}^{\mathbf{L}} S\right)^{\dagger_{E}} \\
& \sim C \otimes_{R}^{\mathbf{L}}\left(C^{\dagger_{D}} \otimes_{R}^{\mathbf{L}} S\right) \\
& \simeq\left(C \otimes_{R}^{\mathbf{L}} \mathbf{R} \operatorname{Hom}_{R}(C, D)\right) \otimes_{R}^{\mathbf{L}} S \\
& \simeq D \otimes_{R}^{\mathbf{L}} S . \quad \square
\end{aligned}
$$


(7.3) Type. For a complex $Z \in \mathcal{D}_{-}^{\mathrm{f}}(R)$ we refer to its first non-vanishing Bass number as its type, that is, type ${ }_{R} Z=\mu_{R}^{\operatorname{depth}_{R} Z}(Z)$, cf. (1.7). For the ring we write type $R=\operatorname{type}_{R} R=\mu_{R}^{\text {depth } R}$. When $\mathrm{fd} \varphi<\infty$ we also write type $\varphi$ for the first non-vanishing coefficient of the Bass series $\mathrm{I}_{\varphi}(t)$, cf. (5.5.1); no confusion arises, as this is in agreement with the more general definition of type $\varphi$ given in [LLAHBF98. (7.1)], cf. [LLAHBF98, (7.8)].

(7.4) Observation. Let $\varphi$ be of finite flat dimension, and assume that $R$ and $S$ have dualizing complexes $D$ and $E$, respectively. Let $C$ be a semi-dualizing complex for $R$. Since $S$ belongs to ${ }_{C} \mathcal{A}(R)$ as well as to ${ }_{C^{\dagger} D} \mathcal{A}(R)$, the pair $\left(C, C^{\dagger_{D}}\right)$ of semi-dualizing complexes for $R$ gives rise to four semi-dualizing complexes for $S$ : namely, the base changed complexes $C \otimes_{R}^{\mathbf{L}} S$ and $C^{\dagger_{D}} \otimes_{R}^{\mathbf{L}} S$, cf. (5.7), and their dagger duals $\left(C \otimes_{R}^{\mathbf{L}} S\right)^{\dagger_{E}}$ and $\left(C^{\dagger_{D}} \otimes_{R}^{\mathbf{L}} S\right)^{\dagger_{E}}$, cf. [2.12). It follows by (5.7) (b) that

$$
\begin{aligned}
\operatorname{type}_{S}\left(C \otimes_{R}^{\mathrm{L}} S\right) & =\operatorname{type}_{R} C \cdot \operatorname{type} \varphi \text { and } \\
\operatorname{type}_{S}\left(C^{\dagger_{D}} \otimes_{R}^{\mathrm{L}} S\right) & =\operatorname{type}_{R} C^{\dagger_{D}} \cdot \operatorname{type} \varphi .
\end{aligned}
$$

By (3.18.3) and (1.7.6) we have

$$
\mathrm{I}_{S}^{\left(C \otimes_{R}^{\mathrm{L}} S\right)^{\dagger} E}(t)=\mathrm{P}_{C \otimes_{R}^{S} S}^{S}(t) t^{e}=\mathrm{P}_{C}^{R}(t) t^{e}=\mathrm{I}_{R}^{C^{\dagger} D}(t) t^{e-d},
$$

for suitable $d, e \in \mathbb{Z}$ and, therefore,

$$
\operatorname{type}_{S}\left(C \otimes_{R}^{\mathbf{L}} S\right)^{\dagger_{E}}=\text { type }_{R} C^{\dagger_{D}} \quad \text { and } \quad \operatorname{type}_{S}\left(C^{\dagger_{D}} \otimes_{R}^{\mathrm{L}} S\right)^{\dagger_{E}}=\operatorname{type}_{R} C .
$$

We know from (7.2) that unless $\varphi$ is Gorenstein, i.e., type $\varphi=1$, the semi-dualizing complexes $\left(C \otimes \otimes_{R}^{\mathbf{L}} S\right)^{\dagger_{E}}$ and $C^{\dagger_{D}} \otimes_{R}^{\mathbf{L}} S$ must be different, and the equations above show that the type is a convenient numerical invariant for telling semi-dualizing complexes apart.

(7.5) Example. Let $\varphi$ be of finite flat dimension, and assume that $D$ and $E$ are dualizing complexes for, respectively, $R$ and $S$. Then we have

$$
\begin{aligned}
\text { type } S & =\text { type } R \cdot \operatorname{type} \varphi, & \operatorname{type}_{S} E & =1, \\
\operatorname{type}_{S}\left(D \otimes_{R}^{\mathrm{L}} S\right) & =\text { type } \varphi, \text { and } & \operatorname{type}_{S} A & =\operatorname{type} R,
\end{aligned}
$$

where $A=\left(D \otimes \otimes_{R}^{\mathbf{L}} S\right)^{\dagger_{E}} \simeq \mathbf{R H o m}_{R}(D, E)$ is dualizing for $\varphi$, cf. (7.1) and (5.1) (c). Thus, if $R$ and $\varphi$ are not Gorenstein, i.e., type $R>1$ and type $\varphi>1$, then $S$ has at least three semi-dualizing complexes of different type, and if type $R \neq$ type $\varphi$, then all the four semi-dualizing complexes above are of different type.

(7.6) Observation. Let $\varphi$ be finite of finite flat dimension, i.e., $S \in \mathcal{P}_{0}^{\mathrm{f}}(R)$, and assume that $\varphi$ is not Gorenstein, that is, type $\varphi>1$. (An example follows below.) Let $C_{1}, \ldots, C_{n}$ be $n \geq 1$ semi-dualizing complexes for $R$ of different type. For each $\ell \in\{1, \ldots, n\}$ the complexes $\mathbf{R} \operatorname{Hom}_{R}\left(S, C_{\ell}\right)$ and $C_{\ell} \otimes_{R}^{\mathbf{L}} S$ are semi-dualizing for $S$ with type $\mathbf{R H o m}_{R}\left(S, C_{\ell}\right)=\operatorname{type}_{R} C_{\ell}$ and type ${ }_{S}\left(C_{\ell} \otimes \mathrm{L}_{R} S\right)=\operatorname{type}_{R} C_{\ell} \cdot \operatorname{type} \varphi$, cf. (6.1) (a) and (5.7)(b). Thus, $S$ will have at least $n+1$ semi-dualizing complexes of different type, namely (assuming that $C_{1}$ has minimal type among $C_{1}, \ldots, C_{n}$ ) the complexes

$$
\mathbf{R H o m}_{R}\left(S, C_{1}\right), C_{1} \otimes_{R}^{\mathbf{L}} S, \ldots, C_{n} \otimes_{R}^{\mathbf{L}} S .
$$


We note that if type $\varphi$ does not divide type $C_{\ell}$ for any $\ell \in\{1, \ldots, n\}$, then the $2 n$ semi-dualizing complexes

$$
\mathbf{R H o m}_{R}\left(S, C_{1}\right), \ldots, \mathbf{R} \operatorname{Hom}_{R}\left(S, C_{n}\right), C_{1} \otimes_{R}^{\mathbf{L}} S, \ldots, C_{n} \otimes_{R}^{\mathbf{L}} S
$$

will all have different type.

(7.7) Example. Consider the trivial extension $S=R \ltimes L$ of $R$ by a free $R$-module $L$. It is a Noetherian local ring with maximal ideal $\mathfrak{m} \times L, \operatorname{dim} S=\operatorname{dim} R$, and $\operatorname{depth} S=\operatorname{depth} R$. Viewed as an $R$-module $S$ is isomorphic to the direct sum $R \oplus L$, so the inclusion map $\varphi: R \rightarrow S$ is a finite flat local homomorphism, and its type is determined by the closed fiber: $\operatorname{type} \varphi=\operatorname{type} S / \mathfrak{m} S$, cf. (5.5). There is an isomorphism of $R$-modules $S / \mathfrak{m} S \cong k \ltimes k^{r}$, where $r$ is the rank of $L$, and it is straightforward to check that it is also an isomorphism of rings. The ring $k \ltimes k^{r}$ is Artinian (because $k$ is so) with maximal ideal $\mathfrak{M}=0 \times k^{r}$. The type of $k \ltimes k^{r}$, and hence of $\varphi$, is the rank of the socle Soc $k \ltimes k^{r}$, which is $r$ as $\mathfrak{M}^{2}=0$. Thus, we have type $\varphi=\operatorname{rank} L$.

Let $C$ be a semi-dualizing complex for $R$. It follows by flatness (freeness) of $S$ over $R$ that the induced semi-dualizing complexes are $C \otimes_{R} S$ and $\operatorname{Hom}_{R}(S, C)$.

(7.8) Golod's Question. In [EGl85] Golod asked for examples of semi-dualizing modules for local rings in addition to the canonical ones, that is, rings and dualizing modules. This question is answered by the example and the observation above:

If $R$ is Cohen-Macaulay, then so are the trivial extensions $S=R \ltimes L$ considered above. It follows from (7.6) that if $\operatorname{rank} L>1$, then the extension $\operatorname{ring} S=R \ltimes L$ will have at least two different semi-dualizing complexes, and by (3.7) these will both be modules. For any finite number $n$ we can, by iterating this procedure, construct a Cohen-Macaulay local ring possessing at least $n$ different semi-dualizing modules.

By (3.18.2) the type of a semi-dualizing complex must divide the type of the ring. Let $R$ be Gorenstein, then type $R=1$, cf. (1.8), and $R$ has exactly one semi-dualizing module (this will be proved in (8.6)). Setting $S_{(1)}=R \ltimes R^{2}$ and recursively defining $S_{(\ell+1)}=S_{(\ell)} \ltimes S_{(\ell)}^{2^{2 \ell}}$, we establish a sequence of Cohen-Macaulay rings, where the $\ell$-th ring has type $S_{(\ell)}=2^{2^{\ell}-1}$ and possesses semi-dualizing modules of each of the possible $2^{\ell}$ different types.

Golod's question has previously (in 1987) been addressed by H.-B. Foxby. Starting with a non-Gorenstein ring $R$, and setting $S=R \ltimes M$ for some finite module $M$ (e.g., $M=R^{2}$ ) with $G-\operatorname{dim}_{R} M=0$ and $\operatorname{Hom}_{R}(M, R)$ non-cyclic, he proved that $\operatorname{Hom}_{R}(S, R)$ is semi-dualizing for $S$ and neither isomorphic to $S$ nor dualizing for $S$.

\section{UNIQUENESS RESUlts}

In this section $R$ is a local ring with residue field $k$.

Foxby duality and dagger duality has already been studied for rings and dualizing complex es, see for example [LLAHBF97], [HHA], [HBF], and [SYs95]. We start by summing up some results from these studies: 
Let $D$ be a dualizing complex for $R$. The next implications are part of the original Foxby duality theorem LLAHBF97, (3.2)], cf. (4.2).

$$
\begin{aligned}
X \in \mathcal{F}(R) & \Longrightarrow D \otimes_{R}^{\mathbf{L}} X \in \mathcal{I}(R) ; \quad \text { and } \\
Y \in \mathcal{I}(R) & \Longrightarrow \operatorname{RHom}_{R}(D, Y) \in \mathcal{F}(R) .
\end{aligned}
$$

For complexes $Z$ in $\mathcal{D}_{\mathrm{b}}^{\mathrm{f}}(R)={ }_{D} \mathcal{R}(R)$ it follows by (3.18.3) that

$$
Z \in \mathcal{P}^{\mathrm{f}}(R) \quad \Longleftrightarrow \quad Z^{\dagger_{D}} \in \mathcal{I}^{\mathrm{f}}(R) .
$$

Foxby duality with respect to $R$ is trivial. As proved in (2.13) dagger duality with respect to $R$ is an endofunctor of $\mathcal{P}^{\mathrm{f}}(R)$, in particular,

$$
Z \in \mathcal{P}^{\mathrm{f}}(R) \quad \Longrightarrow \quad Z^{\dagger_{R}} \in \mathcal{P}^{\mathrm{f}}(R) .
$$

The two main results of this section characterize $R$ and a dualizing complex for $R$ in terms of special properties of their associated dagger and Foxby duality functors. Theorem (8.1) shows that only for $C \sim R$ is the dagger duality functor ${ }^{t^{\dagger}}$ stable on the subcategory $\mathcal{P}^{\mathrm{f}}(R)$ of ${ }_{C} \mathcal{R}(R)$. Theorem (8.2) shows that only if $C$ is dualizing for $R$ do we get genuine Foxby duality, cf. (4.2), meaning that the functors $C \otimes_{R}^{\mathbf{L}}-$ and $\mathbf{R H o m}_{R}(C,-)$ also provide quasi-inverse equivalences of the subcategories $\mathcal{F}(R)$ and $\mathcal{I}(R)$ of ${ }_{C} \mathcal{A}(R)$ and ${ }_{C} \mathcal{B}(R)$.

The proofs of the two theorems are given at the end of the section.

(8.1) Theorem. If $C$ is a semi-dualizing complex for $R$, then the following are equivalent:

(i) $C \sim R$.

(ii) $Z^{\dagger_{C}} \in \mathcal{P}^{\mathrm{f}}(R)$ for some $Z \not 0$ in $\mathcal{P}^{\mathrm{f}}(R)$.

(iii) $C \otimes_{R}^{\mathrm{L}} X \in \mathcal{F}(R)$ for some $X \in{ }_{C} \mathcal{A}(R)$ with $\operatorname{depth}_{R} X<\infty$.

(iii') $\operatorname{depth}_{R} Y<\infty$ for some $Y \in{ }_{C} \mathcal{B}(R) \cap \mathcal{F}(R)$.

(iv) $\mathbf{R H o m}_{R}(C, Y) \in \mathcal{I}(R)$ for some $Y \in{ }_{C} \mathcal{B}(R)$ with $\operatorname{depth}_{R} Y<\infty$.

(iv') $\operatorname{depth}_{R} X<\infty$ for some $X \in{ }_{C} \mathcal{A}(R) \cap \mathcal{I}(R)$.

In (8.1) the second condition should be compared to (8.0.4), and (iii) and (iv) should be compared to (8.0.1) and (8.0.2), respectively.

Conditions (iii) and (iv) in the next theorem show that a dualizing complex can be unveiled by 'a single instance of genuine Foxby duality', cf. (8.0.1) and (8.0.2). The second condition should be compared to (8.0.3).

(8.2) Theorem. If $C$ is a semi-dualizing complex for $R$, then the following are equivalent:

(i) $C$ is dualizing for $R$.

(ii) $Z^{\dagger_{C}} \in \mathcal{I}^{\mathrm{f}}(R)$ for some $Z \not 0$ in $\mathcal{D}_{+}^{\mathrm{f}}(R)$.

(ii') There is a complex $Y \not 0$ in ${ }_{C} \mathcal{R}(R) \cap \mathcal{I}(R)$.

(iii) $C \otimes_{R}^{\mathbf{L}} X \in \mathcal{I}(R)$ for some $X \in \mathcal{F}(R)$ with $\operatorname{depth}_{R} X<\infty$.

(iv) $\mathbf{R H o m}_{R}(C, Y) \in \mathcal{F}(R)$ for some $Y \in \mathcal{I}(R)$ with $\operatorname{depth}_{R} Y<\infty$.

As a first step towards a proof of these theorems we establish two results characterizing semi-dualizing complexes in terms of their Poincaré and Bass series and special elements in their Auslander categories. 
In MJn75 it was proved that a PG-module (see [HBF72]) of finite G-dimension is projective, so over a local ring all $\mathrm{PG}$-modules are free, and $R$ is the unique rank $1 \mathrm{PG}$-module. This result is extended to complexes by (8.3): up to isomorphism and shift, $R$ is the unique semi-dualizing complex in ${ }_{R} \mathcal{R}(R)$, in particular, it is the only semi-dualizing complex of finite projective dimension.

From [RAD, V.3.1] we know that, up to isomorphism and shift, a dualizing complex $D$ is the only semi-dualizing complex of finite injective dimension. From (8.4) it follows that $D$ is also the unique semi-dualizing complex in ${ }_{D} \mathcal{B}(R)$.

(8.3) Proposition. If $C$ is a semi-dualizing complex for $R$, then the following are equivalent:

(i) $C \sim R$.

(ii) $\mathrm{P}_{C}^{R}(t)$ is a monomial.

(iii) $C \in{ }_{R} \mathcal{R}(R)$.

(iv) $k \in{ }_{C} \mathcal{A}(R)$.

(iv') $k \in C_{C} \mathcal{B}(R)$.

(v) ${ }_{C} \mathcal{A}(R)=\mathcal{D}_{\mathrm{b}}(R)$.

$\left(v^{\prime}\right){ }_{C} \mathcal{B}(R)=\mathcal{D}_{\mathrm{b}}(R)$.

(8.4) Proposition. If $C$ is a semi-dualizing complex for $R$, then the following are equivalent:

(i) $C$ is dualizing for $R$.

(ii) $\mathrm{I}_{R}^{C}(t)$ is a monomial.

(iii) $R$ has a dualizing complex $D$, and $C \in{ }_{D} \mathcal{B}(R)$.

(iv) $k \in{ }_{C} \mathcal{R}(R)$.

(v) ${ }_{C} \mathcal{R}(R)=\mathcal{D}_{\mathrm{b}}^{\mathrm{f}}(R)$.

Proof of (8.3). It is well-known that conditions $(i)$ and $(i i)$ are equivalent, cf. (1.7.2), and they certainly imply (iii), cf. (2.8). Also the implications $(i) \Rightarrow(v) \Rightarrow(i v)$ and $(i) \Rightarrow\left(v^{\prime}\right) \Rightarrow\left(i v^{\prime}\right)$ are clear, cf. (4.3).

(iii) $\Rightarrow($ ii $)$ : Assume that $C \in{ }_{R} \mathcal{R}(R)$, then we have

$$
\begin{aligned}
\mathrm{I}_{R}^{C}(t) & =\mathrm{P}_{C^{\dagger} R}^{R}(t) \mathrm{I}_{R}(t) & & \text { by }(3.18 .1 \\
& =\mathrm{P}_{C^{\dagger}}^{R}(t) \mathrm{P}_{C}^{R}(t) \mathrm{I}_{R}^{C}(t) & & \text { by }(3.18 .2)
\end{aligned}
$$

This implies that $\mathrm{P}_{C^{\dagger} R}^{R}(t) \mathrm{P}_{C}^{R}(t)=1$, and since $\mathrm{P}_{C^{\dagger} R}^{R}(t)$ and $\mathrm{P}_{C}^{R}(t)$ are formal Laurant series with non-negative coefficients, we can conclude that they are monomials.

$(i v) \Rightarrow\left(\right.$ iii): If $k \in C \mathcal{A}(R)$ then, in particular, $C \otimes_{R}^{\mathbf{L}} k \in \mathcal{D}_{\mathrm{b}}(R)$ so $\operatorname{pd}_{R} C=$ $\sup \left(C \otimes \otimes_{R}^{\mathbf{L}} k\right)<\infty$, cf. (1.4.4), and hence $C$ is $R$-reflexive.

$\left(i v^{\prime}\right) \Rightarrow(i i i)$ : As we just saw, it is sufficient to prove that $\sup \left(C \otimes_{R}^{\mathbf{L}} k\right)<\infty$; we do so by using Matlis duality $\left(\mathrm{E}_{R}(k)\right.$ is the injective hull of the residue field):

$$
\begin{aligned}
\sup \left(C \otimes_{R}^{\mathbf{L}} k\right) & =-\inf \left(\operatorname{Hom}_{R}\left(C \otimes_{R}^{\mathbf{L}} k, \mathrm{E}_{R}(k)\right)\right) \\
& =-\inf \left(\mathbf{R H o m}_{R}\left(C, \operatorname{Hom}_{R}\left(k, \mathrm{E}_{R}(k)\right)\right)\right) \\
& =-\inf \left(\mathbf{R H o m}_{R}(C, k)\right) \\
& <\infty,
\end{aligned}
$$


as $\operatorname{Hom}_{R}\left(k, \mathrm{E}_{R}(k)\right) \cong k$ and $\mathbf{R H o m}_{R}(C, k) \in \mathcal{D}_{\mathrm{b}}(R)$. This concludes the proof.

Proof of (8.4). The equivalence of conditions (i) and (ii) is well-known, cf. (1.8.1), and they obviously, cf. (4.4) and (2.8), imply (iii) and $(v)$, the latter of which, in turn, implies $(i v)$.

(iii) $\Rightarrow($ ii $)$ : Suppose $D$ is a dualizing complex for $R$ and $C \in{ }_{D} \mathcal{B}(R)$. We may assume that $D$ is normalized, and we find:

$$
\begin{aligned}
& \mathrm{P}_{C}^{R}(t)=\mathrm{P}_{D \otimes_{R}^{\mathrm{L}} \mathbf{R} \operatorname{Hom}_{R}(D, C)}^{R}(t) \\
& =\mathrm{P}_{D}^{R}(t) \mathrm{P}_{\mathbf{R} \operatorname{Hom}_{R}(D, C)}^{R}(t) \quad \text { by (1.7.6) } \\
& =\mathrm{I}_{R}(t) \mathrm{P}_{\mathbf{R} \operatorname{Hom}_{R}(D, C)}^{R}(t) \quad \text { by (3.18.3) } \\
& =\mathrm{P}_{C}^{R}(t) \mathrm{I}_{R}^{C}(t) \mathrm{P}_{\mathbf{R} \operatorname{Hom}_{R}(D, C)}^{R}(t) \text { by (3.18.2). }
\end{aligned}
$$

This implies that $\mathrm{I}_{R}^{C}(t) \mathrm{P}_{\mathbf{R} \operatorname{Hom}_{R}(D, C)}^{R}(t)=1$, and since these Laurant series have non-negative coefficients, we conclude that $\mathrm{I}_{R}^{C}(t)$ is a monomial.

$(i v) \Rightarrow(i)$ : If $k$ belongs to ${ }_{C} \mathcal{R}(R)$ then, in particular, $\mathbf{R H o m}_{R}(k, C) \in \mathcal{D}_{\mathrm{b}}^{\mathrm{f}}(R)$ so $\operatorname{id}_{R} C=-\inf \left(\mathbf{R H o m}_{R}(k, C)\right)<\infty$, cf. (1.4.5) $)$, and $C$ is, indeed, dualizing for $R$.

(8.5) Remarks. Recall that $R$ is said to be Gorenstein if and only if $\operatorname{id}_{R} R<\infty$ or, equivalently, if and only if $R$ is a dualizing complex for $R$, cf. (1.8.2). We note that (8.3) and (8.4) contain the fact that the conditions

(i) $R$ is Gorenstein; (ii) $G-\operatorname{dim}_{R} k<\infty$; and

(iii) $G-\operatorname{dim}_{R} M<\infty$ for all finite $R$-modules $M$;

are equivalent. This result [MAu67, Théorème 3, p. 64] explains the name 'Gorenstein dimension'.

The reader is invited to apply (8.1) to a dualizing complex for $R$ and (8.2) to $R$ and, thereby, obtain a series (with some redundancy) of conditions equivalent with $R$ being Gorenstein. The equivalence of conditions $\left(i i i^{\prime}\right)$ and $\left(i v^{\prime}\right)$ in (8.1) applied to a dualizing complex were originally discovered by Foxby, cf. [HBF]. Further conditions can be extracted from (8.3) and (8.4); we only spell out the following:

(8.6) Corollary. If $D$ is dualizing complex for $R$, then the following are equivalent:

(i) $R$ is Gorenstein.

(ii) $R$ has only one semi-dualizing complex (up to isomorphism and shift).

(iii) $D \sim R$.

(iv) ${ }_{D} \mathcal{A}(R)={ }_{D} \mathcal{B}(R)=\mathcal{D}_{\mathrm{b}}(R)$.

Proof. The equivalence $(i) \Leftrightarrow($ iii) is (1.8.2), and (iii) $\Leftrightarrow(i v)$ follows by (8.3). It follows by (8.4) that $(i v)$ implies (ii), as any semi-dualizing complex will belong to ${ }_{D} \mathcal{B}(R)$, and the implication $(i i) \Rightarrow($ iii $)$ is obvious.

(8.7) Lemma. Let $C$ be a semi-dualizing complex for $R$. For $U \in \mathcal{D}_{\mathrm{b}}(R)$ the following are equivalent:

(i) $\mathbf{R H o m}_{R}(k, U) \not 0$. 
(i') $\operatorname{depth}_{R} U<\infty$.

(ii) $k \otimes_{R}^{\mathbf{L}} U \not 0$.

(iii) $\mathbf{R H o m}_{R}\left(k, \mathbf{R} \operatorname{Hom}_{R}(C, U)\right) \not 0$.

(iv) $k \otimes_{R}^{\mathbf{L}}\left(C \otimes_{R}^{\mathbf{L}} U\right) \not 0$.

Proof. The equivalence of $(i)$ and $\left(i^{\prime}\right)$ is evident by the definition of depth, (1.6.1), and $(i) \Leftrightarrow(i i)$ by HBF79, 2.8]. The complex $C$ has finite homology modules and, therefore, finite depth, so it follows from what we have already proved that $\mathbf{R H o m}_{R}(k, C) \neq 0$ and $k \otimes_{R}^{\mathbf{L}} C \not 0$. The equivalences $(i) \Leftrightarrow(i i i)$ and $(i i) \Leftrightarrow(i v)$ now follow straight from the next two chains of isomorphisms.

$$
\begin{aligned}
\mathbf{R H o m}_{R}\left(k, \mathbf{R H o m}_{R}(C, U)\right) & \simeq \mathbf{R} \operatorname{Hom}_{R}\left(k \otimes_{R}^{\mathbf{L}} C, U\right) \\
& \simeq \mathbf{R H o m}_{R}\left(\left(k \otimes_{R}^{\mathbf{L}} C\right) \otimes_{k} k, U\right) \\
& \simeq \operatorname{Hom}_{k}\left(k \otimes_{R}^{\mathbf{L}} C, \mathbf{R} H_{R}(k, U)\right) ; \text { and } \\
k \otimes_{R}^{\mathbf{L}}\left(C \otimes_{R}^{\mathbf{L}} U\right) & \simeq\left(k \otimes_{R}^{\mathbf{L}} C\right) \otimes_{R}^{\mathbf{L}} U \\
& \simeq\left(k \otimes_{R}^{\mathbf{L}} C\right) \otimes_{k}\left(k \otimes_{R}^{\mathbf{L}} U\right) . \quad \square
\end{aligned}
$$

Proof of (8.1). Clearly, $(i)$ implies (ii), (iii), and (iv).

$($ ii $) \Rightarrow(i)$ : When $Z \in \mathcal{P}^{\mathrm{f}}(R)$ we have $\mathrm{P}_{Z^{\dagger} C}^{R}(t)=\mathrm{P}_{Z}^{R}\left(t^{-1}\right) \mathrm{P}_{C}^{R}(t)$ by (2.14), and hence $\operatorname{pd}_{R} Z^{\dagger_{C}}=\operatorname{pd}_{R} C-\inf Z$, cf. (1.7.3). This shows that if $Z^{\dagger_{C}}$ belongs to $\mathcal{P}^{\mathrm{f}}(R)$, then so does $C$ and hence $C \sim R$ by (8.3).

(iii) $\Rightarrow$ (iii'): Set $Y=C \otimes_{R}^{\mathbf{L}} X$, then $Y \in{ }_{C} \mathcal{B}(R) \cap \mathcal{F}(R)$ by [4.6). Furthermore, $k \otimes_{R}^{\mathbf{L}} Y \not 40$, by (8.7), and hence $\operatorname{depth}_{R} Y<\infty$, as $Y \in \mathcal{D}_{\mathrm{b}}(R)$.

(iii') $\Rightarrow$ (i): Suppose $Y \in{ }_{C} \mathcal{B}(R) \cap \mathcal{F}(R)$ has $\operatorname{depth}_{R} Y<\infty$. By (8.7) we have $\mathbf{R H o m}_{R}\left(k, \mathbf{R} \operatorname{Hom}_{R}(C, Y)\right) \not 00$ and, again by (8.7), $k \otimes_{R}^{\mathbf{L}} \mathbf{R} \operatorname{Hom}_{R}(C, Y) \neq 0$; in particular, $-\sup \left(k \otimes_{R}^{\mathbf{L}} \mathbf{R} \operatorname{Hom}_{R}(C, Y)\right)<\infty$. Now, by (4.5) (c) we have

$$
Y \otimes_{R}^{\mathbf{L}} k \simeq\left(C \otimes_{R}^{\mathbf{L}} k\right) \otimes_{R}^{\mathbf{L}} \mathbf{R H o m}_{R}(C, Y) \simeq\left(C \otimes_{R}^{\mathbf{L}} k\right) \otimes_{k}\left(k \otimes_{R}^{\mathbf{L}} \mathbf{R H o m}_{R}(C, Y)\right),
$$

and hence

$$
\sup \left(Y \otimes_{R}^{\mathbf{L}} k\right)=\sup \left(C \otimes_{R}^{\mathbf{L}} k\right)+\sup \left(k \otimes_{R}^{\mathbf{L}} \mathbf{R} \operatorname{Hom}_{R}(C, Y)\right) .
$$

By (1.4.4) and (1.4.1) we have

$$
\begin{aligned}
\operatorname{pd}_{R} C=\sup \left(C \otimes_{R}^{\mathbf{L}} k\right) & =\sup \left(Y \otimes_{R}^{\mathbf{L}} k\right)-\sup \left(k \otimes_{R}^{\mathbf{L}} \mathbf{R} \operatorname{Hom}_{R}(C, Y)\right) \\
& \leq \operatorname{fd}_{R} Y-\sup \left(k \otimes_{R}^{\mathbf{L}} \mathbf{R} \operatorname{Hom}_{R}(C, Y)\right) \\
& <\infty,
\end{aligned}
$$

and it follows by (8.3) that $C \sim R$.

$(i v) \Rightarrow\left(i v^{\prime}\right)$ : Set $X=\mathbf{R H o m}_{R}(C, Y)$, then $\operatorname{depth}_{R} X<\infty$ by (8.7), and $X \in$ ${ }_{C} \mathcal{A}(R) \cap \mathcal{I}(R)$ by (4.6) .

$\left(i v^{\prime}\right) \Rightarrow(i)$ : If $X \in{ }_{C} \mathcal{A}(R) \cap \mathcal{I}(R)$ has $\operatorname{depth}_{R} X<\infty$, then, still applying (8.7), we see that $\mathbf{R H o m}_{R}\left(k, C \otimes \otimes_{R}^{\mathbf{L}} X\right) \not \varkappa 0$; in particular, $\inf \left(\mathbf{R H o m}_{R}\left(k, C \otimes \otimes_{R}^{\mathbf{L}} X\right)\right)<\infty$. By (4.5) (a) we have

$$
\begin{aligned}
\operatorname{RHom}_{R}(k, X) & \simeq \mathbf{R H o m}_{R}\left(C \otimes_{R}^{\mathbf{L}} k, C \otimes_{R}^{\mathbf{L}} X\right) \\
& \simeq \mathbf{R} \operatorname{Hom}_{R}\left(\left(C \otimes_{R}^{\mathbf{L}} k\right) \otimes_{k} k, C \otimes_{R}^{\mathbf{L}} X\right) \\
& \simeq \operatorname{Hom}_{k}\left(C \otimes_{R}^{\mathbf{L}} k, \mathbf{R H o m}\right. \\
& \left.\left(k, C \otimes_{R}^{\mathbf{L}} X\right)\right),
\end{aligned}
$$


and it follows that

$$
\inf \left(\mathbf{R H o m}_{R}(k, X)\right)=\inf \left(\mathbf{R H o m}_{R}\left(k, C \otimes_{R}^{\mathbf{L}} X\right)\right)-\sup \left(C \otimes_{R}^{\mathbf{L}} k\right) .
$$

Hence, by (1.4.4) and (1.4.2),

$$
\begin{aligned}
\operatorname{pd}_{R} C=\sup \left(C \otimes_{R}^{\mathbf{L}} k\right) & =\inf \left(\mathbf{R H o m}_{R}\left(k, C \otimes_{R}^{\mathbf{L}} X\right)\right)-\inf \left(\mathbf{R H o m}_{R}(k, X)\right) \\
& \leq \inf \left(\mathbf{R H o m} \operatorname{Hom}_{R}\left(k, C \otimes_{R}^{\mathbf{L}} X\right)\right)+\operatorname{id}_{R} X \\
& <\infty ;
\end{aligned}
$$

and the desired result again follows by (8.3).

Proof of (8.2). Certainly, (i) implies (ii'), and also (iii) and (iv), cf. (8.0.1) and (8.0.2)

$\left(i i^{\prime}\right) \Rightarrow(i i)$ : Set $Z=Y^{\dagger_{C}}$.

$($ ii $) \Rightarrow(i)$ : By (1.7.7) we have $\mathrm{I}_{R}^{Z^{\dagger} C}(t)=\mathrm{P}_{Z}^{R}(t) \mathrm{I}_{R}^{C}(t)$ and therefore $\operatorname{id}_{R} Z^{\dagger_{C}}=$ $\operatorname{pd}_{R} Z+\operatorname{id}_{R} C$; cf. (1.7.1) and (1.7.3). Thus, $Z^{\dagger_{C}} \in \mathcal{I}^{\mathrm{f}}(R)$ implies that $C \in \mathcal{I}^{\mathrm{f}}(R)$ (and $Z \in \mathcal{P}^{\mathrm{f}}(R)$ ), and it follows that $C$ is, in fact, dualizing for $R$.

$($ iii $) \Rightarrow(i)$ : It is sufficient to prove that $\operatorname{id}_{R} C<\infty$. It follows by (1.5.7) that

$$
\begin{aligned}
\inf \left(\mathbf{R H o m}_{R}\left(k, C \otimes_{R}^{\mathbf{L}} X\right)\right) & =\inf \left(\mathbf{R H o m}_{R}(k, C) \otimes_{R}^{\mathbf{L}} X\right) \\
& =\inf \left(\mathbf{R H o m}_{R}(k, C) \otimes_{k}\left(k \otimes_{R}^{\mathbf{L}} X\right)\right) \\
& =\inf \left(\mathbf{R H o m}_{R}(k, C)\right)+\inf \left(k \otimes_{R}^{\mathbf{L}} X\right) .
\end{aligned}
$$

Hence,

$$
\begin{aligned}
-\inf \left(\mathbf{R H o m}_{R}(k, C)\right) & =\inf \left(k \otimes_{R}^{\mathbf{L}} X\right)-\inf \left(\mathbf{R} \operatorname{Hom}_{R}\left(k, C \otimes_{R}^{\mathbf{L}} X\right)\right) \\
& \leq \inf \left(k \otimes_{R}^{\mathbf{L}} X\right)+\operatorname{id}_{R}\left(C \otimes_{R}^{\mathbf{L}} X\right) \\
& <\infty
\end{aligned}
$$

where the inequalities follow by (1.4.2), (8.7), and the assumptions on $X$. Since $\operatorname{id}_{R} C=-\inf \left(\mathbf{R} \operatorname{Hom}_{R}(k, C)\right)$ by (1.4.5) $)$, we are done.

$(i v) \Rightarrow(i)$ : As above it is sufficient to prove that $-\inf (\mathbf{R H o m} R(k, C))<\infty$. It follows by (1.5.8) that

$$
\begin{aligned}
\sup \left(k \otimes_{R}^{\mathbf{L}} \mathbf{R H o m}_{R}(C, Y)\right) & =\sup \left(\mathbf{R H o m}_{R}\left(\mathbf{R H o m}_{R}(k, C), Y\right)\right) \\
& =\sup \left(\mathbf{R} \operatorname{Hom}_{R}\left(\mathbf{R} \operatorname{Hom}_{R}(k, C) \otimes_{k} k, Y\right)\right) \\
& =\sup \left(\operatorname{Hom}_{k}\left(\mathbf{R} \operatorname{Hom}_{R}(k, C), \mathbf{R H o m} R(k, Y)\right)\right) \\
& =\sup \left(\mathbf{R H o m}_{R}(k, Y)\right)-\inf \left(\mathbf{R H o m}_{R}(k, C)\right) .
\end{aligned}
$$

Hence, by (1.4.1) and the assumptions on $Y$, we have

$$
\begin{aligned}
-\inf \left(\mathbf{R H o m}_{R}(k, C)\right) & =\sup (\mathbf{R H o m} \\
& \left.\leq \operatorname{fd}_{R} \mathbf{R} \operatorname{Hom}_{R}(C, Y) \otimes_{R}^{\mathbf{L}} k\right)-\sup \left(\mathbf{R H o m}_{R}(k, Y)\right) \\
& <\infty . \quad \square
\end{aligned}
$$

\section{Appendix. Chain Defects}

Throughout the appendix $R$ is a local ring.

The inequality

$$
\operatorname{cmd} R \leq \operatorname{amp} C+\operatorname{cmd}_{R} C
$$


holds for every semi-dualizing complex for $R$; this was established in (3.4) (c). Obviously, equality holds if $C$ is dualizing for $R$ or $C \sim R$, and it was established ibid. that equality holds if $C$ is Cohen-Macaulay or $\operatorname{amp} C=0$. The purpose of this appendix is to show that the difference between the two sides in $(\ddagger)$ is governed by (differences in) so-called catenary defects of $C$ and $R$. Thus, the main result is (A.5), but our approach is more general, cf. A.4.

(A.1) Definitions. Let $Z \in \mathcal{D}_{\mathrm{b}}^{\mathrm{f}}(R)$. For $\mathfrak{p} \in \operatorname{Supp}_{R} Z$ we consider the two integers:

$$
\begin{aligned}
\operatorname{dimd}_{R}(\mathfrak{p}, Z) & =\operatorname{dim}_{R} Z+\inf Z_{\mathfrak{p}}-\operatorname{dim} R / \mathfrak{p} ; \quad \text { and } \\
\operatorname{catd}_{R}(\mathfrak{p}, Z) & =\operatorname{dim}_{R} Z-\operatorname{dim}_{R_{\mathfrak{p}}} Z_{\mathfrak{p}}-\operatorname{dim} R / \mathfrak{p} .
\end{aligned}
$$

Both numbers belong to $\mathbb{N}_{0}$ by, respectively, the definition of dimension, (1.6.2), and (1.6.5). The number $\operatorname{catd}_{R}(\mathfrak{p}, Z)$ is the catenary defect of $Z$ at $\mathfrak{p}$. See also the appendix in [LLAHBF98], where these numbers are related to certain conjectures of M. Auslander.

(A.2) Observation. Let $Z \in \mathcal{D}_{\mathrm{b}}^{\mathrm{f}}(R)$ and $\mathfrak{p} \in \operatorname{Supp}_{R} Z$; by (1.6.3) and the definitions above we have

$$
0 \leq \operatorname{dimd}_{R}(\mathfrak{p}, Z)-\operatorname{catd}_{R}(\mathfrak{p}, Z)=\inf Z_{\mathfrak{p}}+\operatorname{dim}_{R_{\mathfrak{p}}} Z_{\mathfrak{p}} \leq \operatorname{dim} R_{\mathfrak{p}}
$$

(A.3) Definition. Let $C$ be a semi-dualizing complex for $R$. For $Z \in{ }_{C} \mathcal{R}(R)$ we consider the integer

$$
\Delta_{C}(Z)=\sup \left\{\operatorname{catd}_{R}(\mathfrak{p}, C)-\operatorname{catd}_{R}(\mathfrak{p}, Z) \mid \mathfrak{p} \in \operatorname{Supp}_{R} Z\right\} .
$$

Note from (A.2) that $\operatorname{dimd}_{R}(\mathfrak{p}, Z)=0 \operatorname{implies} \operatorname{catd}_{R}(\mathfrak{p}, Z)=0$, and hence $\Delta_{C}(Z) \geq$ 0 .

(A.4) Theorem. Let $C$ be a semi-dualizing complex for $R$. For $Z \in{ }_{C} \mathcal{R}(R)$ there is an inequality:

$$
\operatorname{cmd}_{R} Z \leq \operatorname{amp} Z^{\dagger_{C}}+\operatorname{cmd}_{R} C-\Delta_{C}(Z) ;
$$

and for $Z \in \mathcal{P}^{\mathrm{f}}(R)$ also the next inequality holds:

$$
\operatorname{amp} Z^{\dagger_{C}}+\operatorname{cmd}_{R} C-\Delta_{C}(Z) \leq \operatorname{cmd}_{R} Z+\operatorname{amp} Z \text {. }
$$

In particular, for $M \in \mathcal{P}_{0}^{\mathrm{f}}(R)$ there is an equality:

$$
\operatorname{cmd}_{R} M=\operatorname{amp} M^{\dagger_{C}}+\operatorname{cmd}_{R} C-\Delta_{C}(M) .
$$

The proof of the theorem is found at the end of the section; applying part (c) to $R \in{ }_{C} \mathcal{R}(R)$ we get the following:

(A.5) Corollary. If $C$ is a semi-dualizing complex for $R$, then

$$
\text { cmd } R=\operatorname{amp} C+\operatorname{cmd}_{R} C-\Delta_{C}(R) \text {. }
$$

(A.6) Remark. Let $C$ be a semi-dualizing complex for $R$. It follows by (3.3) (a) and (A.4) (a) that

$$
0 \leq \Delta_{C}(Z) \leq \operatorname{cmd}_{R} C \text { for } Z \in{ }_{C} \mathcal{R}(R) .
$$

The proof of A.4 requires two lemmas and a proposition; the first lemma has the following interesting consequence: 
(A.7) Corollary (to A.8). Let $C$ be a semi-dualizing complex for $R$, and set $s=$ $\sup C$. For $\mathfrak{p} \in \operatorname{Spec} R$ the next biconditional holds:

$$
\mathfrak{p} \in \operatorname{Ass}_{R} \mathrm{H}_{s}(C) \Longleftrightarrow \operatorname{depth} R_{\mathfrak{p}}=0 \wedge \sup C=\inf C_{\mathfrak{p}} ;
$$

and there is an equality:

$$
\operatorname{dimd}_{R}(\mathfrak{p}, C)-\operatorname{catd}_{R}(\mathfrak{p}, C)=\operatorname{depth} R_{\mathfrak{p}}+\operatorname{cmd}_{R_{\mathfrak{p}}} C_{\mathfrak{p}} .
$$

(A.8) Lemma. Let $C$ be a semi-dualizing complex for $R$, and let $Z$ be $C$-reflexive. The following hold for $\mathfrak{p} \in \operatorname{Supp}_{R} Z$ :

$$
\begin{aligned}
\inf Z_{\mathfrak{p}}+\operatorname{depth}_{R_{\mathfrak{p}}} Z_{\mathfrak{p}} & =\operatorname{dimd}_{R}(\mathfrak{p}, Z)-\operatorname{catd}_{R}(\mathfrak{p}, Z)-\operatorname{cmd}_{R_{\mathfrak{p}}} Z_{\mathfrak{p}} \\
& =\inf \left(Z^{\dagger_{C}}\right)_{\mathfrak{p}}+\operatorname{depth}_{R_{\mathfrak{p}}}\left(Z^{\dagger_{C}}\right)_{\mathfrak{p}} ; \quad \text { and } \\
\inf Z_{\mathfrak{p}}+\operatorname{depth}_{R_{\mathfrak{p}}} Z_{\mathfrak{p}} & \geq \inf \left(Z^{\dagger_{C}}\right)_{\mathfrak{p}}-\sup Z^{\dagger_{C}} .
\end{aligned}
$$

Equality holds in (b) if and only if $\mathfrak{p} \in \operatorname{Ass}_{R} \mathrm{H}_{s}\left(Z^{\dagger_{C}}\right)$ for $s=\sup Z^{\dagger_{C}}$.

Proof. (a) follows by the next computation:

$$
\begin{array}{rlrl}
\operatorname{dimd}_{R}(\mathfrak{p}, Z)-\operatorname{catd}_{R}(\mathfrak{p}, Z) & -\operatorname{cmd}_{R_{\mathfrak{p}}} Z_{\mathfrak{p}} & \\
& =\inf Z_{\mathfrak{p}}+\operatorname{dim}_{R_{\mathfrak{p}}} Z_{\mathfrak{p}}-\operatorname{cmd}_{R_{\mathfrak{p}}} Z_{\mathfrak{p}} & & \text { by } \\
& =\inf Z_{\mathfrak{p}}+\operatorname{depth}_{R_{\mathfrak{p}}} Z_{\mathfrak{p}} & \\
& =\inf Z_{\mathfrak{p}}+\operatorname{depth}_{R_{\mathfrak{p}}} C_{\mathfrak{p}}+\inf \left(Z^{\dagger}\right)_{\mathfrak{p}} & & \text { by (3.1)(a) } \\
& =\operatorname{depth}_{R_{\mathfrak{p}}}\left(Z^{\dagger_{C}}\right)_{\mathfrak{p}}+\inf \left(Z^{\dagger_{C}}\right)_{\mathfrak{p}} & & \text { by (3.1)(a). }
\end{array}
$$

(b) follows from (a) as $\operatorname{depth}_{R_{\mathfrak{p}}}\left(Z^{\dagger_{C}}\right)_{\mathfrak{p}} \geq-\sup Z^{\dagger_{C}}$ with equality if and only if $\mathfrak{p} \in \operatorname{Ass}_{R} \mathrm{H}_{s}\left(Z^{\dagger_{C}}\right)$ for $s=\sup Z^{\dagger_{C}}$, cf. (1.6.6).

Proof of A.7. Let $\mathfrak{p} \in \operatorname{Spec} R$ and recall that by (3.2) (a) we have $\operatorname{depth}_{R_{\mathfrak{p}}} C_{\mathfrak{p}}+$ $\inf C_{\mathfrak{p}}=\operatorname{depth} R_{\mathfrak{p}}$. The equality now follows by applying (A.8) (a) to $Z=C$ and the biconditional by applying (A.8) (b) to $Z=R$.

(A.9) Lemma. Let $C$ be a semi-dualizing complex for $R$, and let $Z$ be $C$-reflexive. For $\mathfrak{p} \in \operatorname{Supp}_{R} Z$ there is an inequality:

$$
\operatorname{catd}_{R}(\mathfrak{p}, C)-\operatorname{catd}_{R}(\mathfrak{p}, Z) \leq \operatorname{dim}_{R} C-\operatorname{dim}_{R} Z+\sup \left(Z^{\dagger_{C}}\right)_{\mathfrak{p}} ;
$$

and if $\operatorname{cmd}_{R_{\mathfrak{p}}} Z_{\mathfrak{p}} \geq \operatorname{cmd}_{R_{\mathfrak{p}}} C_{\mathfrak{p}}$ also the next inequality holds:

$$
\operatorname{dim}_{R} C-\operatorname{dim}_{R} Z+\inf \left(Z^{\dagger_{C}}\right)_{\mathfrak{p}} \leq \operatorname{catd}_{R}(\mathfrak{p}, C)-\operatorname{catd}_{R}(\mathfrak{p}, Z) .
$$

Proof. Both inequalities follow by straightforward computations.

$$
\begin{aligned}
\operatorname{catd}_{R}(\mathfrak{p}, C)-\operatorname{catd}_{R}(\mathfrak{p}, Z) & =\operatorname{dim}_{R} C-\operatorname{dim}_{R} Z-\operatorname{dim}_{R_{\mathfrak{p}}} C_{\mathfrak{p}}+\operatorname{dim}_{R_{\mathfrak{p}}} Z_{\mathfrak{p}} \\
& \leq \operatorname{dim}_{R} C-\operatorname{dim}_{R} Z+\sup \left(Z^{\dagger_{C}}\right)_{\mathfrak{p}} ;
\end{aligned}
$$

the inequality follows by (3.1) (b).

$$
\text { (b): } \begin{aligned}
\operatorname{catd}_{R}(\mathfrak{p}, C)-\operatorname{catd}_{R}(\mathfrak{p}, Z) \geq & \operatorname{dim}_{R} C-\operatorname{dim}_{R} Z-\operatorname{dim}_{R_{\mathfrak{p}}} C_{\mathfrak{p}}+\operatorname{dim}_{R_{\mathfrak{p}}} Z_{\mathfrak{p}} \\
& +\operatorname{cmd}_{R_{\mathfrak{p}}} C_{\mathfrak{p}}-\operatorname{cmd}_{R_{\mathfrak{p}}} Z_{\mathfrak{p}} \\
= & \operatorname{dim}_{R} C-\operatorname{dim}_{R} Z-\operatorname{depth}_{R_{\mathfrak{p}}} C_{\mathfrak{p}}+\operatorname{depth}_{R_{\mathfrak{p}}} Z_{\mathfrak{p}} \\
= & \operatorname{dim}_{R} C-\operatorname{dim}_{R} Z+\inf \left(Z^{\dagger_{C}}\right)_{\mathfrak{p}} ;
\end{aligned}
$$

the last equality is (3.1)(a). 
The next proposition extends and generalizes [LLAHBF98, (A.4)]

(A.10) Proposition. Let $C$ be a semi-dualizing complex for $R$. For $Z \in{ }_{C} \mathcal{R}(R)$ there is an inequality:

$$
\Delta_{C}(Z) \leq \operatorname{dim}_{R} C-\operatorname{dim}_{R} Z+\sup Z^{\dagger_{C}}
$$

and for $Z \in \mathcal{P}^{\mathrm{f}}(R)$ there are also inequalities:

$$
\begin{aligned}
\Delta_{C}(Z) & \geq \operatorname{dim}_{R} C-\operatorname{dim}_{R} Z+\sup \left\{\inf \left(Z^{\dagger_{C}}\right)_{\mathfrak{p}} \mid \mathfrak{p} \in \operatorname{Supp}_{R} Z\right\} \\
& \geq \operatorname{dim}_{R} C-\operatorname{dim}_{R} Z+\sup Z^{\dagger_{C}}-\operatorname{amp} Z .
\end{aligned}
$$

In particular, the next equality holds for $M \in \mathcal{P}_{0}^{\mathrm{f}}(R)$ :

$$
\Delta_{C}(M)=\operatorname{dim}_{R} C-\operatorname{dim}_{R} M+\sup M^{\dagger_{C}} .
$$

Proof. (a) is an immediate consequence of (A.9) (a).

(b): Suppose $Z \in \mathcal{P}^{\mathrm{f}}(R) \subseteq{ }_{C} \mathcal{R}(R)$. For each $\mathfrak{p} \in \operatorname{Spec} R$ the complex $C_{\mathfrak{p}}$ is semi-dualizing for $R_{\mathfrak{p}}$ and $Z_{\mathfrak{p}} \in \mathcal{P}^{\mathrm{f}}\left(R_{\mathfrak{p}}\right) \subseteq C_{\mathfrak{p}} \mathcal{R}\left(R_{\mathfrak{p}}\right)$, so by (3.4) (b) and (3.6.1) we have inequalities $\mathrm{cmd}_{R_{\mathfrak{p}}} C_{\mathfrak{p}} \leq \mathrm{cmd} R_{\mathfrak{p}} \leq \operatorname{cmd}_{R_{\mathfrak{p}}} Z_{\mathfrak{p}}$. The first inequality now follows by A.9 (b). To prove the second set $s=\sup Z^{\dagger_{C}}$, choose $\mathfrak{q}$ in $\operatorname{Ass}_{R} \mathrm{H}_{s}\left(Z^{\dagger_{C}}\right)$ and recall that $\mathfrak{q} \in \operatorname{Supp}_{R} Z^{\dagger_{C}}=\operatorname{Supp}_{R} Z$ by 3.1 (d). We now have

$$
\begin{array}{rlr}
\sup \left\{\inf \left(Z^{\dagger_{C}}\right)_{\mathfrak{p}} \mid \mathfrak{p} \in \operatorname{Supp}_{R} Z\right\} & \geq \inf \left(Z^{\dagger_{C}}\right)_{\mathfrak{q}} \\
& =\sup Z^{\dagger_{C}}+\inf Z_{\mathfrak{q}}+\operatorname{depth}_{R_{\mathfrak{q}}} Z_{\mathfrak{q}} & \text { by (1.8) (b) } \\
& \geq \sup Z^{\dagger_{C}}+\inf Z_{\mathfrak{q}}-\sup Z & \text { by (1.6.4) } \\
& \geq \sup Z^{\dagger_{C}}-\operatorname{amp} Z ;
\end{array}
$$

and this proves the desired inequality.

Finally, (c) follows from (a) and (b) as amp $M=0$.

Proof of (A.4. By (A.10) we have inequalities:

$$
\operatorname{dim}_{R} Z \leq \operatorname{dim}_{R} C+\sup Z^{\dagger_{C}}-\Delta_{C}(Z)
$$

for $Z \in{ }_{C} \mathcal{R}(R)$; and

$$
\operatorname{dim}_{R} C+\sup Z^{\dagger_{C}}-\Delta_{C}(Z) \leq \operatorname{dim}_{R} Z+\operatorname{amp} Z
$$

for $Z \in \mathcal{P}^{\mathrm{f}}(R)$. The inequalities (a) and (b) now follow by 3.1) (a), and (c) is a consequence of (a) and (b).

\section{REFERENCES}

[MAu67] Maurice Auslander, Anneaux de Gorenstein, et torsion en algèbre commutative, Secrétariat mathématique, Paris, 1967, Séminaire d'Algèbre Commutative dirigé par Pierre Samuel, 1966/67. Texte rédigé, d'après des exposés de Maurice Auslander, par Marquerite Mangeney, Christian Peskine et Lucien Szpiro. École Normale Supérieure de Jeunes Filles. MR 37:1435

[MAuMBr69] Maurice Auslander and Mark Bridger, Stable module theory, American Mathematical Society, Providence, R.I., 1969, Memoirs of the American Mathematical Society, No. 94. MR 42:4580

[LLAGBF91] Luchezar L. Avramov and Hans-Bjørn Foxby, Homological dimensions of unbounded complexes, J. Pure Appl. Algebra 71 (1991), no. 2-3, 129-155. MR 93g:18017

[LLAHBF92] _ Locally Gorenstein homomorphisms, Amer. J. Math. 114 (1992), no. 5, 1007-1047. MR 93i:13019 
[LLAHBF97] _ Ring homomorphisms and finite Gorenstein dimension, Proc. London Math. Soc. (3) 75 (1997), no. 2, 241-270. MR 98d:13014

[LLAHBF98] _ Cohen-Macaulay properties of ring homomorphisms, Adv. Math. 133 (1998), no. 1, 54-95. MR 99c:13043

[LLAHBFHr94] Luchezar L. Avramov, Hans-Bjørn Foxby, and Bernd Herzog, Structure of local homomorphisms, J. Algebra 164 (1994), no. 1, 124-145. MR 95f:13029

[HBs63] Hyman Bass, On the ubiquity of Gorenstein rings, Math. Z. 82 (1963), 8-28. MR 27:3669

[LWC0] Lars Winther Christensen, Gorenstein dimensions, Master's thesis, Matematisk Institut, Københavns Universitet, 1996.

[EJX96] Edgar E. Enochs, Overtoun M. G. Jenda, and Jinzhong Xu, Foxby duality and Gorenstein injective and projective modules, Trans. Amer. Math. Soc. 348 (1996), no. 8, 3223-3234. MR 96k:13010

[HBF] Hans-Bjørn Foxby, Auslander categories, in preparation.

[HHA] _ Hyperhomological algebra 83 commutative rings, notes in preparation.

[HBF72] _ Gorenstein modules and related modules, Math. Scand. 31 (1972), 267-284 (1973). MR 48:6094

[HBF75] , Quasi-perfect modules over Cohen-Macaulay rings, Math. Nachr. 66 (1975), 103-110. MR 51:12838

[HBF77] - Isomorphisms between complexes with applications to the homological theory of modules, Math. Scand. 40 (1977), no. 1, 5-19. MR 56:5584

[HBF79] _ Bounded complexes of flat modules, J. Pure Appl. Algebra 15 (1979), no. 2, 149-172. MR 83c: 13008

[HBF80] _ Homological dimensions of complexes of modules, Séminaire d'Algèbre Paul Dubreil et Marie-Paule Malliavin, 32ème année (Paris, 1979), Springer, Berlin, 1980, pp. 360368. MR 82a:13001

[HBFAT77] Hans-Bjørn Foxby and Anders Thorup, Minimal injective resolutions under flat base change, Proc. Amer. Math. Soc. 67 (1977), no. 1, 27-31. MR 56:11984

[EGl84] Evgeniy S. Golod, G-dimension and generalized perfect ideals, Trudy Mat. Inst. Steklov. 165 (1984), 62-66, Algebraic geometry and its applications. MR 85m:13011

[EGl85] — Uspekhi Mat. Nauk 40 (1985), no. 1(241), 234, (Question, in Russian).

[RAD] Robin Hartshorne, Residues and duality, Springer-Verlag, Berlin, 1966, Lecture notes of a seminar on the work of A. Grothendieck, given at Harvard 1963/64. With an appendix by P. Deligne. Lecture Notes in Mathematics, No. 20. MR 36:5145

[MHc75] Melvin Hochster, Topics in the homological theory of modules over commutative rings, Published for the Conference Board of the Mathematical Sciences by the American Mathematical Society, Providence, R.I., 1975, Expository lectures from the CBMS Regional Conference held at the University of Nebraska, Lincoln, Neb., June 24-28, 1974, Conference Board of the Mathematical Sciences Regional Conference Series in Mathematics, No. 24. MR 51:8096

[BIv77] Birger Iversen, Amplitude inequalities for complexes, Ann. Sci. École Norm. Sup. (4) 10 (1977), no. 4, 547-558. MR 58:27966

[MJn75] M. I. Jinnah, A note on PG-modules, Math. Scand. 37 (1975), no. 1, 27-28. MR 52:8107

[CPsLSz73] Christian Peskine and Lucien Szpiro, Dimension projective finie et cohomologie locale. Applications à la démonstration de conjectures de M. Auslander, H. Bass et A. Grothendieck, Inst. Hautes Études Sci. Publ. Math. (1973), no. 42, 47-119. MR 51:10330

[PRb76] Paul Roberts, Two applications of dualizing complexes over local rings, Ann. Sci. École Norm. Sup. (4) 9 (1976), no. 1, 103-106. MR 53:2926

[PRb87] — Le théorème d'intersection, C. R. Acad. Sci. Paris Sér. I Math. 304 (1987), no. 7, 177-180. MR 89b:14008

[NSp88] Nicolas Spaltenstein, Resolutions of unbounded complexes, Compositio Math. 65 (1988), no. 2, 121-154. MR 89m:18013

[JVe77] Jean-Louis Verdier, Catégories dérivées. quelques résultats (état 0), SGA $4 \frac{1}{2}$, Springer, Berlin Heidelberg New York, 1977, pp. 262-311.

[JXu96] Jinzhong Xu, Flat covers of modules, Springer-Verlag, Berlin, 1996. MR 98b:16003

[SYs95] Siamak Yassemi, G-dimension, Math. Scand. 77 (1995), no. 2, 161-174. MR 97d:13017

Matematisk Afdeling, Universitetsparken 5, DK-2100 Copenhagen $\varnothing$, Denmark.

E-mail address: winther@math.ku.dk 